\title{
Attention and Adaptation \\ IN \\ Visual Motion Processing
}

\author{
Dissertation \\ zur Erlangung des Doktorgrades \\ der Mathematisch-Naturwissenschaftlichen Fakultäten \\ der Georg-August Universität zu Göttingen
}

vorgelegt von

DIPL. BIOL.

FLORIAN PIEPER

aus Brake / Unterweser

Göttingen 2007 
D 7

Referent: Prof. Dr. Stefan Treue

Korreferent: Prof. Dr. Ralf Heinrich

Tag der mündlichen Prüfung: 30. Oktober 2007 
Es könnt' alles so einfach sein,...

... isses aber nicht!

\section{Fanta 4}





\section{Acknowledgments}

During the last years, Stefan Treue promoted me to acquire numerous experiences on the many different aspects of vision, neurosciences and computer-programming. I want to thank him for all this support. My time in his lab has been and will always remain an enlightening epoch in my life.

I'd also like to express my gratitude to Prof. Heinrich who agreed to be the second reviewer of this thesis. Thank you Prof. Uwe Jürgens and Prof. Peter Kappeler to be part of my examining board and for the cooperative, confident collaborations during the last years.

Thilo Womelsdorf: Thank you for your help and ever-present motivational support when things did not proceed as expected. I will never forget that and will always be indebted to you. Thank you Julio Cesar Martínez-Trujillo for the valuable discussions, help and fun that we had.

Pinar Boyraz was an excellent office-mate. To all my other colleagues, Katharina Anton-Erxleben, Daniel Kaping, Tzvetomir Tzvetanov, Steffen Katzner, Laura Busse, Alexander Gail, Ralf Rode-Brockhausen, Stefanie Schuldes, Anja Lochte, Vladislav Kozirev, Beatrix Glaser, ...: Thanks for your support and nice lunch-times during the last years. Dirk Prüsse did a great job as 'monkey-master' and was always supporting. Sabine Stuber made the lab a nice place to be and her powerfully eloquent emails were the refreshing brainteasers of the day.

Thanks also to Ulrike, Joachim and the other 'Studt- und Neumänner', who always let me feel welcomed. ("Am besten wäre,...")

Without my parents love and support throughout my entire life I would not be, who I am: Thank you, Helga and Hans-Hermann for all! The 'Renzelmann-Clan' always lets me know, where my roots are. You are great!

Marlenna, you and Carl Joshua have the incredible ability to convert also middling days to good days and fill my life with sunshine. Thank you so much! 


\section{Preface}

The question about what man and animals perceive and how they come to their decisions is ever present in the history of man. To understand the mechanisms of perception and deciding, it is thus essential to have an idea about what the ultimate aims of decisions and the natural limitations of neuronal tissue are.

\section{Basics of decision}

According to actual biological encyclopedia, life has - besides others - two basic attributes: homeostasis and metabolism. To maintain homeostasis, already the single cell is confronted with the problem of what substance to allow to pass the membrane in either direction. The living cell thus has to recognize a substance and implicitly make an assumption about the future 'value' of the newly reached 'state', i.e. after its passage through the membrane. The decision should be optimized to maximize reproductability. In principle, larger animals with a dedicated nervous systems and a muscular apparatus to execute movements are faced with the same problem. They are permanently (!) deciding, if and how to change their interaction with the environment to reach or maintain a reproductive status. Also they have to predict the future and draw inferences about what might be a successful behavior given the current constellation of the environment and the 'self'. In summary, decisions may be expressed a kind of Bayesian estimation upon the -'historical'- knowledge and actual environment with the information about past and present represented ('stored') in the nervous system.

\section{What delimits information storage and processing?}

In his book "What is life?" (1944) the physicist and Nobel-laureate Erwin Schödinger defined life, according to the 2nd law of thermodynamics, as a process that builds on the local decrease of entropy (negentropy) in a universe which itself constantly increases entropy and disgregation. Locale entropy decrease as such can

only take place at the cost of an at least equal entropy increase, i.e., energy dissipation, somewhere else, e.g. the sun. 
The representation ,i.e. 'replication', of environmental information in the subjects nervous system is such an entropy reducing process and hence costs energy. On the other hand, in principle, maximizing the amount of information that is available to the subject, should help it to come to better and better decisions based on a more reliable prediction of the future by extrapolating from the past. Life thus has to compromise between saving energy and increasing the amount of information used for biologically adaptive conclusions.

\section{Making he most of a given brain}

One structural limitation of every subject is the amount of neuronal tissue and thus neurons, it can 'refer to'. At any instance in time the amount of information in the outer world is much higher than what can enter the subject via its sense organs and what can be processed by the brain. The sensors and the neurons are hence filters in many dimensions (e.g. in the case of vision: space, luminance, contrast, color, orientaion, direction of motion,...). The sensitivity, selectivity and bandwidth of such filters determines, what information reaches perception and is available for decision making. In total, one can conclude that the brain's representation of the world is not complete with respect to what information is truly present in the 'outer' world.

However, besides the genetical limitations of the amount of neuronal tissue, there exist cellular and network-mechanisms that allow for 'inlife', experience and concept-based adaptation of the information flow and hence variability of decision making. This means, given an equal physical input, a change in the perception, decisions and behavior may happen due to an 'adapted' evaluation of this input based upon 'knowledge' that itself is subject to change on different time-scales and mechanisms.

This work will put its focus on some variable, knowledge-based information processing and perception due to adaptation to the sensory ('bottom-up') history and ('top-down') attention - as expression of expectation - in the primate visual system:

How and how far are the filters in our heads subject to changes in their sensitivity, selectivity and bandwidth and how does this affect our perception? 




\section{Contents}

1 Introduction 1

1.1 Fundamentals of the visual system in primates . . . . . . . . . . 2

1.1.1 Neurons of the visual motion sensitive middle temporal area (MT) ...................... 5

1.1.2 MT activity and its relation to perception . . . . . . . 9

1.2 Variability of visual perception and processing . . . . . . . . . . 11

1.3 Visual adaptation . . . . . . . . . . . . . . . 12

1.3.1 Adaptation effects on visual perception . . . . . . . . . 12

1.3.2 Neuronal correlates of visual Adaptation . . . . . . . . . . 14

1.4 Visual attention . . . . . . . . . . . . . . . . . . 19

1.4.1 Attentional effects on visual perception . . . . . . . . . 19

1.4.2 Neuronal correlates of visual attention . . . . . . . . . 21

2 Brief motion adaptation causes repulsive aftereffects Prepared for submission

3 Response saturation determines attentional modulation onset in MT neurons - Prepared for submission.

4 Dynamic shifts of visual receptive fields in cortical area MT by spatial attention - 'Nature Neuroscience', 9, 9,(2006)

5 Summary

Bibliography

6 Curriculum Vitae 


\section{Chapter 1}

\section{Introduction}

The current work combines a selection of three scientific articles (one published and two submitted for publication) that address the same global question: How temporally variable is the sensory processing of visual information and hence perception due to variable cognitive influences (attention) or the history of the stimulation (adaptation)? Two studies examine the attentional modulation of single cortical neurons which are involved in visual motion processing another study focuses on the psychophysics of adaptation of the visual motion system.

The visual motion processing system of primates is highly developed and basically manifold investigated through psychophysical and neurophysiological studies in both, humans and monkeys. In the past, this system was mostly analyzed with the implicit assumption that it would reside in specific fixed states at any point in time. The brain was assumed to 'switch' from, say, 'passive viewing' to 'with attention' or to 'adapted'. This 'fixed-state' view recently starts to crumble and researchers become more and more interested in the succession and interplay of how the brain 'applies' its mechanisms to manage information processing with changing goals and historical influences.

During the rest of this chapter I will briefly introduce the neuronal basics of vision and visual motion processing and give an insight into the different kinds of adaptational and attentional modification.

In the second part of this thesis I will report the original research articles. Every article will be separately introduced there. 


\subsection{Fundamentals of the visual system in primates}

The retina translates the optical information of the world into visual information of the brain. In the color-sensitive cones and luminance-sensitive rods, electromagnetic waves ranging from about $400 \mathrm{~nm}$ to $700 \mathrm{~nm}$ cause the intracellular receptorpotential to hyperpolarisate, what in turn evokes action potentials in downstream retinal neurons. Because a single retinal receptor receives only information from a limited spatial region of the outer world, it is only able to code temporal changes of luminance, i.e. amplitude fluctuations. Two-dimensional, spatial visual information appears not until the activity of many independent receptors is converging and interacting in the network of horizontal, bipolar, and amacrine cells of the retina. This computation cause different spiking activities in the retina's ganglion-cells, depending on the extend and spatial distribution of light in the visual world. I.e., a cell may respond maximally if light falls only on a specific patch of the retina, namely the cells 'receptive field' (RF), but fails to produce spikes if also the surrounding of its RF is illuminated. Beside these so-called 'on-center' ganglion cells, their functional opponent, the 'off-center' ganglion cells also exist. Together they form the first neuronal stage of spatial visual information. This center-surround antagonism is a common mechanism throughout the downstream visual brain areas.

The axons of two major types of ganglion cells leave the eyes: magnocellular parasol ('M-pathway') and parvocellular midget ('P-pathway') cells. Parasol cells code transient changes of the visual input like in visual motion; midget cells transfer information about color and orientation. A third, recently discovered ganglion cell type is the bistratified cell ('K-pathway'), that exhibits no center-surround antagonism and is color-sensitive to blue ('on') and red/green ('off'). Together they project through the optic nerve and optic tract to the the thalamic lateral geniculate nucleus (LGN). On their path, the branches of each optic nerve split up in the optic chiasm. The axons that have their origin in the retinas nasal half of each eye cross to the contralateral side of the brain, while the temporal half of each retina projects to the ipsilateral side of the brain. Each post-chiasmatic optic tract only translates information from the contralateral visual hemifield from both eyes, what determines the spatial segregation of left and right visual field to the right and left brain hemispheres, respectively. Additionally, about $10 \%$ of the eyes information is directly transferred through separate ganglion cell-types to the superior colliculi, the pretectum and the suprachiasmatic nucleus to guide reflexive eye-movements, accomodation of the pupil and maintain circadian rhythms, respectively. 
The thalamic LGN is the obligatory relay station for all higher vision and has a 6-layerd structure. Retinal M-cells terminate in layer 1 and 2 (ventral) which themself contain large ('magnocellular') neurons, and the P-cells project to layers 3-6 (dorsal) with their parvocellular neurons. Besides the structure of circular RFs, the anatomical segregation between the two eyes' input is also preserved. The contralateral eyes' axonal terminals are in the layers 1,4, and 6; the ipsilateral ones are in the layers 2,3, and 5. The bistratified retinal ganglion cells terminate on the koniocellular ('K') LGN cells with a very small cell body. However, the sum of retinal input makes only about $10 \%$ of the LGNs total input. The exact function of the LGN is still nebulous. Via the optic radiation the LGN projects to the primary visual cortex (V1) in a retinotopic way.

In area V1, also termed striate cortex, the magnocellular output of the LGN reaches layer $4 \mathrm{C} \alpha$ and the parvocellular projection terminate in layer $4 \mathrm{C} \beta$. Not only is the anatomical segregation of the pathways preserved, also the ocular dominance is still present is V1. This is, information from the two eyes reaches different cells in V1 and converges from there on disparity selective binocular cells. New information is extracted through the convergence of cells with concentric RFs and their facilitating and inhibitory interaction in the two main functional cell-types of area V1: simple and complex cells (Hubel and Wiesel, 1962, 1968). Simple cells' RFs have distinctive excitatory and inhibitory sub-regions which can be considered garbor-filter like Figure 1.1, hence they are selective to oriented lines. Complex cells, though also selective for orientations in their RF, miss such distinct spatial fields of activation and inhibition. Moreover, they react to oriented lines everywhere inside their RF and are thus also motion sensitive. The spatial extend of both celltypes RFs is increased compared to the retinas or LGN RF sizes. This is an ubiquitous phenomenon that is also preserved along the extrastriate visual pathways: The spatial information converges to larger and larger spatial RF.

The extrastriate visual pathway consists of two anatomically separated streams (Felleman and Van Essen, 1991). This goes along with a strong but not complete (Ferrera et al., 1994a,b) functional separation: in the ventral pathways input is passed from V1 through area V2 before it enters V4 in the temporal cortex. V4 cells represent color and orientation. The information is further forwarded to areas TEO and IT with its sensitivity for complex shapes that are composed of many basic 'features'. Faces represent very complex objects and are represented in the most 'distal' part of the ventral stream, namely the anterior temporal sulcus (i.e. 


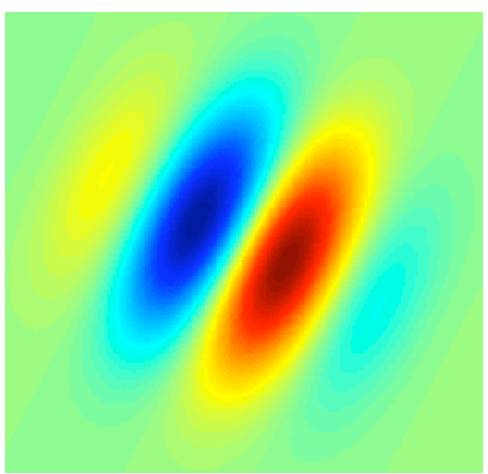

Figure 1.1: Garbor-like receptive field of an orientation selective V1 simple-cell. Red and blue indicate response-activating and response-suppressing sub-regions of the RF. This cell fires best, if the stimulus contains two adjacent bright (right) and a dark (left) components that are orientated roughly at $30^{\circ}$ clockwise. The horizontal and vertical extent is about $1^{\circ} \times 1^{\circ}$. A Garbor is - mathematically - the convolution of two 2D-funtions: An oriented sinosoidal luminance grating is -point by point - multiplied with a circular 2-dimensional gaussian bell-like inverted transparency function. The point of inflection of the bell is located on the edge between the lumince changes. Central parts of the grating are highly effective while to the edges the stimulus energy more and more decreases to background luminance. 
Tsao et al., 2003). The spatial RF size of these cells can span a whole hemifield and may also reach into the ipsilateral hemifield. The ventral stream, also called the 'what'-path is involved in object recognition.

The dorsal - occipito-parietal - pathway, in contrast, is also called the 'where'path, because spatial and spatio-temporal relationships are coded here. Information flows from V1 over V2 and V3 to areas MT and MST. MT and MST neurons are both selective for directions of visual motion, i.e. spatial displacement of 'objects' in time. MT prefers motion in the visual plane, whereas MST is more responsive to optic flow, i.e., motion with a component that is orthogonal to the visual plane and causes the impression of an approximating or departing object.

\subsubsection{Neurons of the visual motion sensitive middle temporal area $(\mathbf{M T})$}

Area MT of the macaque monkey (Macaca mulatta) is located in the upper, lateral bank of the superior temporal sulcus (STS) of the macaque brain. Its human homologue is termed V5 or MT+. MT receives its most prominent inputs from layer 4B-neurons of V1, the thick stripes of area V2 and from area V3. Dubner and Zeki (1971) were the first who reported the direction-selectivity of primates MT neurons. This is, the cells responded to a certain direction $(\theta)$ of visual motion, say, upward motion, with a high spiking rate, decreased this rate gradually for further (counter-) clockwise directions and reached a lower limiting firing rate for downward motion. The neuron's so-called 'preferred direction' is the one that elicits the highest firing rate; the 'null direction' evokes the lowest response and points exactly opposite to the preferred direction $\left(\theta_{\text {null }}^{\circ}=\theta_{\text {pref }}^{\circ}-180^{\circ}\right)$. The circular gaussian approximation (Bratschelet, 1981) (Eqn.:1.1; Fig.:1.2) with only 4 free parameters provides, in general, the best fit to the real direction specific responses (Swindale, 1998):

$$
R(\theta)=B+\sum_{i=-2}^{2} A^{\left(\frac{-(\theta-\phi-i * 360)^{2}}{\sigma^{2}}\right)}
$$

Such, the response $R$, given the presence of direction $\theta$, results from adding the baseline or spontaneous response $B$, to the actual height $A$ of the tuning curve. The parameters $\phi$ and $\sigma$ are the preferred direction and tuning width, which is $\sim 45^{\circ}-55^{\circ}$ (half-width at half-height) in typical MT-neurons. However, a handfull of other functions are available and used by different investigators to fit the responses of MT neurons to moving stimuli (see Swindale, 1998, for comparisons). 
Although the terminology of the different fitting functions in similar, their mathematical behavior and effects of parameter changes differ. This may be one reason for possible discrepancies in the results and their interpretations between different studies that use tuning-properties and -changes according to adaptational and attentional influences (see sections 1.3.2 and 1.4.2).

The direction selectivity of MT neurons as calculated by the directional index (DI) (Eqn.:1.2) is in general larger than 0.3 and can even reach values in the range of 0.98 , which equals a response ratio of $10000 \%$.

$$
D I=\frac{\text { Response }_{\text {pref. }}-\text { Response }_{\text {null }}}{\text { Response }_{\text {pref. }}+\text { Response }_{\text {null }}}
$$

Area MT inherits the retinotopic organization of V1, i.e., neighboring MT patches represent neighboring spatial regions of the visual field (Dubner and Zeki, 1971; Allman and Kaas, 1976; Huk et al., 2002). MT neurons' RFs are 10-fold larger than V1 receptive fields and have a diameter that is roughly equal to their excentricity (factor: 0.8; Maunsell and Van Essen (1983); Britten and Heuer (1999)). The spatial integration of responses from upstream areas evoked by spatially separated stimuli inside the RF acts in a nonlinear way (Britten and Heuer, 1999). This is in some disagreement with a model of (Simoncelli and Heeger, 1998), who proposed a linear intercation. They also introduced a divisive normalization mechanism: the response of each neuron is divided by the total response of the pool that includes other neurons as well as itself. The normalization factor is thus always greater than the cells - non-normalized - response. Hence, its neural response can - mathematically - only vary between ' 0 ' and '1' (i.e. a minimum and a maximum firing rate). This implicates, that the neuron can run (almost) into saturation, i.e. a further increase of the input will not or only minimal increase the output (see chapter 3 ).

Direction selectivity is already present in V1 complex cells. What then is added in MT? It was proposed that MT codes the motion of large objects, that exceed the size of V1 RFs and have individual components that move in different directions. Because V1 RFs are small, they are faced with the aperture problem: V1 cells can only report velocities of contours that are orthogonal to their preferred orientation (velocity is the vector representation of the direction and speed). However, to extract the motion direction of an object, in theory, at least two local motion components need to be combined. The term intersection of constraints (IOC) denotes this geometrical solution. Movshon et al. (1985) tested if MT could solve the aperture problem by presenting plaid stimuli that were composed of two gratings that moved at 


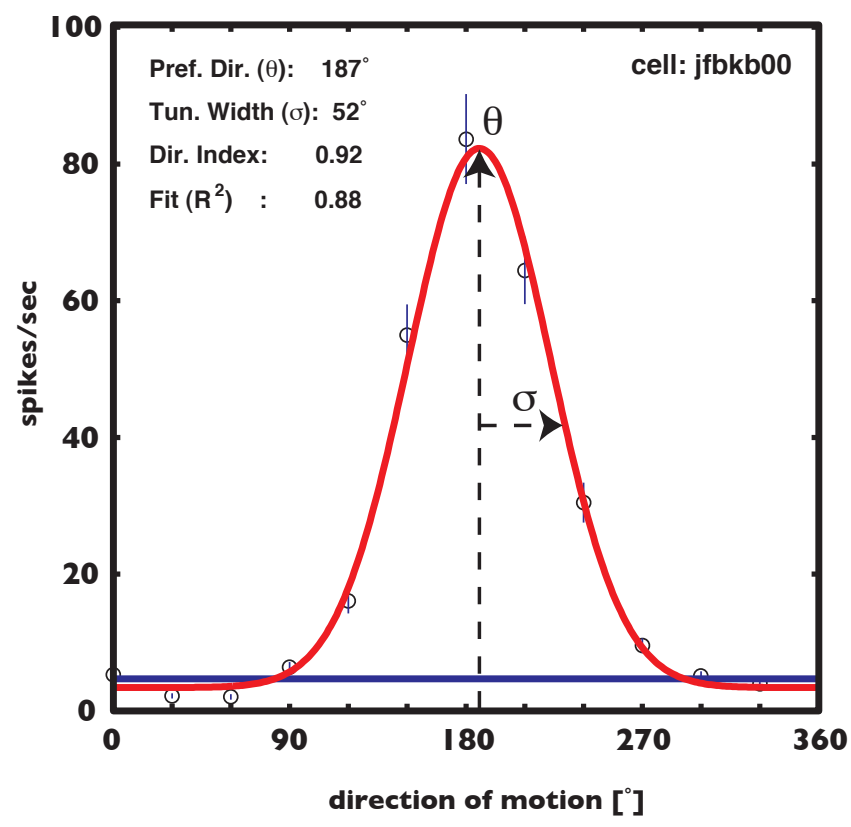

Figure 1.2: Circular gaussian fit (red; Eqn.:1.1) through direction specific responses of a MT neuron. The cell responses with different spike-rates to the presence of different directions of motion ( $30^{\circ}$ separated) in its receptive field. Circles and vertical lines indicate the mean and standard error of the cells response at tested directions of motion. The peak of the fit is at $187^{\circ}$ ( $\sim$ downwards) and determines the preferred direction $(\theta)$ of the cell. The tuning width parameter $(\sigma)$ equals $52^{\circ}$. This cell has a directional selectivity (directional index) of 0.92 as evaluated by Eqn.:1.2. Without any stimulus the cell fires about 5 spikes per second (blue), which is also termed baseline response. The parameter $R^{2}$ refers to the goodness-of-fit of the model equation, fitted to the actual data with a least-square-regression. 
an angle of about $120^{\circ}$. Human subjects reported the perception of a motion pattern that appeared to move in the vector average direction of both components, namely $\pm 60^{\circ}$ apart, respectively. About $25 \%$ of the MT cells showed such a pattern selectivity, while $\sim 40 \%$ were component selective. This means, they responded maximal if either of the two components moved in their preferred direction but showed an intermediate response, if both components moved $60^{\circ}$ apart from the preferred direction. The remaining cells exhibited indifferent preferences. Importantly, none of the also tested V1 cells were pattern selective. However, recent investigations of V1 neurons also revealed some pattern selectivity there (Tinsley et al., 2003; Guo et al., 2004). Movshon et al. (1985) proposed a two-stage model, where the objects components are initially represented in V1 and are then integrated by the second stage (MT) to represent the whole objects velocity. Simoncelli and Heeger (1998) formalized this idea with a computational model of MT: The Fourier transform of any translational pattern is thought to lay a on plane in frequency plane. The pattern velocity is computed as the weighted sum of V1 spatio-temporal oriented complex cells over this plane in frequency space. This planar summation for velocity selectivity is then an implementation of the IOC solution.

Analog to the orientation representation in area V1, MT exhibits a columnar organization: Neurons of similar directional preference form elongated clusters $(\oslash: \sim$ $2 \mathrm{~mm}$ ) perpendicular to the cortical surface (Albright et al., 1984; Liu and Newsome, 2003).

Motion is a 2-dimensional feature, that is, direction and speed are entangled and cannot exist independently. MT neurons also represent the speed of moving objects. Most neurons are sensitive to 'intermediate' speeds of about $1^{\circ}-64^{\circ}$ (Perrone and Thiele, 2001; Ditterich et al., 2003; Priebe et al., 2003; Liu and Newsome, 2003). Hence, many neurons act like a band-pass filter of speed, but also low and high-pass filtering neurons exist. Speed tuning is organized on an anatomical finer scale as direction columns, namely in patches (not columns) that change preferences about every 300-600 $\mu \mathrm{m}$ (Liu and Newsome, 2003). However, Priebe et al. (2003) also reports that a large number of MT neurons is more likely tuned to spatio-temporal frequencies, if tested with a moving grating. The cells respond indistinguishably to, say, a 1 cycle $/{ }^{\circ}$ grating that moves with a speed of $2^{\circ} / \mathrm{sec}$. and to a $2 \mathrm{cycle} /{ }^{\circ}$ grating with $1^{\circ} / \sec$.

The response latencies of MT neurons in awake monkeys are in the range of $70 \pm 10 \mathrm{~ms}$ for flashed high contrast stimuli in the preferred direction; reducing the 
contrast, changing the direction of motion, varying the type of stimuli or the animal's state (awake vs anesthetized) affect the latency (Schmolesky et al., 1998; Bair et al., 2002) such that it becomes longer. Already these first few spikes within the $\sim 100 \mathrm{~ms}$ after stimulus onset carry about $80 \%$ of the direction information (Osborne et al., 2004; Müller et al., 2001). That is, on average, it is possible to reconstruct the presented direction of motion from the first few spikes of a given single neuron. Longer inspection of the stimulus only adds marginal more reliability in the evaluation of that stimulus' direction. But collecting more spikes over a longer period increases the ability to discriminate two different directions of motion (Osborne et al., 2004) from the MT neurons response.

\subsubsection{MT activity and its relation to perception}

Functional exclusion of brain structures, e.g. by lesioning or cooling, can reveal its 'normal' contribution to perception. Lesioning area MT results in considerable elevations of discrimination thresholds for the direction of moving stimuli with no impairment in the processing of stimulus contrast (Newsome and Pare, 1988). Additionally, measures for sensitivity of individual neurons in MT could account for the behavioral performance in a direction discrimination task (Britten et al., 1992) and stimulus detection task (Thiele et al., 2000).

However, at any given point in time, numerous neurons are simultaneously active, representing a given stimulus. Because this is not energy-efficient the brain should take some advantage from coding the same stimulus feature with multiple neurons: First, the responses of individual neurons suffer from noise. That is, they respond differently from trial to trial of equivalent stimulation. Such, the perception should not rely on single neurons, but instead 'combine' the response of several neurons to overcome the noise. Second, from the bell-shaped individual neurons tuning curve it follows that the response of a whole population of neurons, spanning the $360^{\circ}$ direction space, is also a bell-shaped gaussian. I.e., neurons that prefer the actual direction of motion respond maximally, while the response of neurons that prefer other directions is given by the distance of their preferred direction to the actual stimulus' direction and the width of their tuning curve. As mentioned in section 1.1.1, the average half tuning-width of MT neurons is about $\sim 45^{\circ}-55^{\circ}$. Hence, the population of MT neurons has the same tuning-width. However, from that alone it is undecidable if the motion percept is determined by the cell with the highest response in the population (i.e. winner-take-all model) or if perception is 
generated from averaging the responses of all neurons (vector-average model). The latter model is based on the principle of labeled lines. Each neuron is thought to be 'labeled' with its preferred direction. Activated by a stimulus, each direction tuned neuron will vote for its labeled direction. This vote, in turn, will be weighted by the firing rate of the neuron and the perceived direction will correspond to the weighted average of all labels.

Different approaches were used to test the two models. First, researchers used microstimulation techniques in MT to shift monkeys perception/decisions during tasks where they had to judge the direction of an ambiguous motion stimulus. Such stimuli have no net direction of motion and are known as counterphase gratings (spatially fixed amplitude oscillations of luminance) or $0 \%$-coherent motion (random dot pattern (RDP) with each dot moving in a random direction) stimulus. Microstimulation of MT cells with a certain direction preference effected the animals directional judgement of an ambiguous motion stimulusin favor to this direction. It thus acts as if the truly presented motion coherence into that direction would be increased (Salzman et al., 1990; Salzman and Newsome, 1994). In refined experiments monkeys were asked to judge the direction of truly directional stimuli during microstimulation (Groh et al., 1997; Nichols and Newsome, 2002). In summary, for small angular differences between the 'presented' and 'stimulated' direction, the monkeys judged the stimuli to move somewhere between these direction. This is in accordance with a vector-average model. However, when the angular distance of the two 'stimulations' (sensory and electrical) differed by as much as $140^{\circ}$ and more, the monkeys switched to categoric answers, how as if a winner-take-all mechanism would underly his choice. This is, they choose either of the two directions. Unfortunately, during these experiments it is not clear, what the monkeys have actually perceived, i.e. if their decisions on the direction were accompanied with a real percept of that direction.

Treue et al. (2000) used another visual phenomenon to test what might underly the direction perception. The ambiguous neuronal representation induced by the combination of two 'stimuli' (one visual, one electrical) in the microstimulationstudies can also be achieved with two truly visual stimuli, namely transparently moving RDPs. These RDPs are $100 \%$ coherent, i.e., all dots of a layer move in the same direction. Moreover, these two spatially completely overlapping stimuli are perceived by humans as two separate 'surfaces', if their difference in direction exceeds about $10^{\circ}$. However, the population response of MT neurons recorded with these stimuli in their RF stays single-peaked until the directions of the two stimuli 
diverge by roughly $90^{\circ}$, i.e. the width of a prototypical MT neurons' tuning curve. The percept thus cannot be explained by the outcome of a simple vector-average nor a winner-take-all algorithm. The first would not allow to perceive transparently moving stimuli at all, because all input would be 'merged' into one vector (= percept); the second could only detect transparency, if both directions would differ more than $90^{\circ}$ such that the population would consist of two peaks. Accordingly, Treue et al. (2000) proposed a new mechanism that decides about transparency on the basis of the actual width of the population response. If the latter exceeds the standard tuning width by a certain amount, the brain infers, that two surfaces are present.

The debate, however, about numerous additional models of population codes, that have been evolved during the last years (e.g., Pouget et al. (2000); Sanger (2003); Latham and Nirenberg (2005); Ma et al. (2006)) is still great. In summary, these models propose some conditional, i.e., task specific, readout of the populations' response. Depending on the subjects aims, different sub-populations of MT are recruited, to draw the appropriate inference. Purushothaman and Bradley (2005) recently reported some evidence for such a strategy in MT: during a fine $\left( \pm 3^{\circ}\right.$ from vertical) direction discrimination task the behavioral performance of a monkey was best correlated to neuronal responses of cells ('choice probability') that preferred directions about $\sim 60^{\circ}$ away from vertical. Information theory revealed, that indeed these neurons should be the most informative for an observer to solve the rather hard task. Indiscriminate pooling of all MT responses yielded far worse performances than that of the monkey. This strongly suggests, that the animal must have selectively read out cells with the appropriate tuning.

\subsection{Variability of visual perception and processing}

Visual perception is highly variable. The same physical scene or object may be judged differently from one view to another. Psychophysical experiments revealed, that human subjects decide ambiguously in visual detection task with one mandatory answer out of two alternative possibilities ('two-alternative-forced-choice';2-AFC). When faced with a barely visible stimuli, the subjects may either detect or ignore it from trial to trail. This shows, that the neuronal system suffers from noise, that distorts the original signal present in the outer world; which in turn means that visual perception per se is variable and not the outcome of a fixed, machine-like 
calculation.

Besides this noise-variability due to spike-rate fluctuations, also other forms of variability in visual perception exist. These may be termed as contextual variabilities. The term 'context' denotes several stimulus and behavioral dimensions: e.g. it can describe spatial relationships of simultaneously present 'objects' in the visual scene. Several perceptual effects of spatial context are known in different featuredimensions ${ }^{1}$.

A very known example of illusory percepts resulting from the spatial composition of stimuli in the visual scene exist in the orientation-domain: the tilt-illusion (O'Toole and Wenderoth (1977), see also Akiyoshi (2007) for review). A vertical test-grating, if presented alone, is perceived as such. But as soon as an appropriately, say, $\sim 15^{\circ}$ clockwise tilted grating is displayed as an annulus around the test, the same still truly vertical grating is illusory perceived as being tilted slightly counterclockwise. This effect is largest, when the annulus directly attaches the to be judged stimulus and gets smaller with an increasing gap between the two stimuli Wenderoth and Johnstone (1988). This is direct evidence for the spatial extend of lateral interactions between the neurons in the visual cortex (see Schwartz et al., 2007, for review).

In summary, besides noise variations, the spatial composition of a visual scene critically determines the perception of its parts and the background. This thesis manly focusses on two additional aspects of contextual perception. First, in the temporal dimension, the effects of adaptation caused by the history of the visual inputs and, second, task specific, i.e. expectancy driven, contexts of 'voluntary' attention. Both will be introduced in more detail in the next sections.

\subsection{Visual adaptation}

\subsubsection{Adaptation effects on visual perception}

Visual adaptation is an unvoluntary process that reversibly re-adjusts the operational range of the sensory processing chain to the 'recent' environmental conditions. It alows us to reliably preceive changes of the environment across orders of magni-

\footnotetext{
${ }^{1}$ Throughout this thesis the term feature-dimension is used to denote different aspects of a visual stimulus, i.e., luminance, color, orientation, motion, etc.. The term feature alone denotes a particular value of a stimulus dimension. For example, the colors red and green represent two different features.
} 
udes. This is most striking when comparing the average luminance at dusk $\left(10^{-2} \mathrm{~cd} /\right.$ $\mathrm{m}^{2}$ ) with that at high noon $\left(10^{5} \mathrm{~cd} / \mathrm{m}^{2}\right)$. This fast reversibility is the distinction to other forms of other history dependent adaptive changes in the brain, e.g. behavioral imprinting or - to a lesser degree - learning.

The definition 'recent' is somewhat arbitrary and covers a wide range in the literature about adaptation. The range of adaptation durations spans from milliseconds, seconds, hours, to sometimes even days (see Mather, 1998, for reviews). However, nowadays, visual adaptation is usually induced with adaptation durations in the range of hundreds of milliseconds up to minutes. The temporal protocol of the succession of adaptors has also great variability. The presentation of 'adaptors' and to-be-judged test-stimuli may alternate or initial long adaptors may be followed-up by shorter, so called top-up adaptors of the same feature/object which then alternate with test-stimuli. The test-stimuli are then 'misperceived' or 'misjudged' compared to their unadapted inspection.

A fundamental perceptual effect of visual adaptation with a high-contrast stimulus (e.g., grating), is the reduction of contrast sensitivity (e.g., Blakemore and Campbell, 1969a,b). This effect gradually diminishes with further distance between the adaptor's and test-stimulus' feature. I.e. in the motion dimension, after adaptation to a certain direction, this directions detection threshold is increased. However, with increasing angular differences the adaptors direction and the tests direction, the detection threshold decreased to 'normal', that is, the unadapted state, at $\sim 45^{\circ}-55^{\circ}$ (Hol and Treue, 2001).

In addition to the increase of detection thresholds, adaptation causes illusory perceptual distortions of supra-threshold stimuli. These effects are also often termed aftereffects and are present in different feature-dimensions (e.g. shape aftereffects (Suzuki, 2001) or face aftereffects (Leopold et al., 2001, 2005; Webster et al., 2004)).

A very prominent one is the motion aftereffect (MAE) that was already described by Aristoleles (see in Verstraten (1996)) and is today - according to Wohlgemuth (1911) - usually termed waterfall-illusison. Wohlgemuth (1911) reported, that after he had intensively inspected a waterfall - with its downward motion content - for a longer time-period and then directed his gaze to a stationary part of the environment, e.g. the rocks next to the waterfall, he had the impression of an upward motion of the rocks (see Mather, 1998, for reviews).

The strength of motion adaptation can be determined in different ways: in historical investigations the effect size of a MAE, subjects reported the - rather subjective - duration of their illusory percept. Then, researchers switched to a more objec- 
tive titration method to find the necessary amount of motion energy in an otherwise ambiguous motion stimulus (e.g. counterphase grating or $0 \%$-coherent RDP) to counterbalance the MAE (McCarthy, 1993).

Another effect of motion adaptation is the direction aftereffect (DAE) which is expressed in the overestimation of the angular difference of the direction of motion between an adaptor and a successively presented 100\%-coherently moving test stimulus. This effect is largest for a true difference of about $45^{\circ}$ (Levinson and Sekuler, 1976; Schrater and Simoncelli, 1998) and will be explained in more detail in one of the papers (Chapter: 2) of this thesis. The DAE has its equivalent in the orientationdomain: the tilt-aftereffect (TAE) (Gibson and Radner, 1937; Gibson, 1937; Sekuler and Littlejohn, 1974). It refers to the overestimation of the angular differences of oriented lines. Here the maximum effect is obtained with an adaptor-test difference of about $15-20^{\circ}$,i.e., roughly half the value of the DAE. The double-angle representation of motion $\left(360^{\circ}\right)$ compared to orientation $\left(180^{\circ}\right)$ (Clifford et al., 2000; Clifford, 2002) would predict such a relationship. Therefore it is intensely debated if similar neuronal mechanisms may underly both adaptation effects (Clifford et al., 2000).

Additionally, also the perception the second dimension of motion, namely speed, is affected by adaptation: After prolonged inspection of a motion pattern its speed appears to be reduced (Clifford and Wenderoth, 1999; Krekelberg et al., 2006; Thompson, 1981; Schrater and Simoncelli, 1998). Clifford and Wenderoth (1999) and Krekelberg et al. (2006) also reported an increased discriminability of speeds in the range of the adaptors speed.

Finally, it has to be mentioned that the effects of motion adaptation can persist a 'back-calibration', if the subjects close their eyes immediately after the adapting procedure (see Mather, 1998). When they re-open the eyes after a delay after which the MAE is usually diminished with constantly open eyes, the MAE is still there. This was long taken as evidence against a simple 'fatigue' or exhaustion-based theory of adaptation (see section 1.3.2), because a 'recovery' should even happen faster without stimulation. Recently, van de Grind et al. (2004) proposed that a still image - viewed after adaptation and that evokes neuronal activity in the visual areas - also acts as a new adaptor, that may actively 'pull back' the perceptual bias and hence increases the speed of 'recovery' compared to waiting with closes eyes.

Theoretical hypotheses about adaptation and its perceptual consequences predict two effects of adaptation. First, a shift of the center in the feature-space, i.e., what is assumed to be 'normal' or 'new standard' (Barlow, 1961; Barlow and Földiak, 
1989). This is expressed by the abovementioned shift of the detection threshold or the new point of subjective equality in a direction-biased ambiguous motion stimulus after directional adaptation. Second, theory also predicts for a variation of the operational range based on the statistics of the current visual input. This, in turn, could increase the discriminability 'around' the adaptor and should affect the tuning-width of sensory neurons. However, this is rarely reported (e.g.: Krekelberg et al., 2006) in the psychophysical literature.

In summary, despite the preposition 'mis-' for many perceptual consequences of adaptation the adaptive re-calibration mechanism is thought to bias the sensitivity of the perceptual system in favor to detect discrepancies in the environment from the new' norm'.

\subsubsection{Neuronal correlates of visual Adaptation}

An established method to indirectly obtain information about the different dendritic and spiking activities of neurons by means of their metabolism is the functional magnetic resonance imaging (fMRI) (Logothetis et al., 2001). It measures the haemodynamic responses, i.e the blood oxygenation level (BOLD) caused by the changes in the amount of the tissues activity. In the human cortex, motion adaptation causes specific reductions of MT+ activity in the fMRI signal (He et al., 1998; Culham et al., 1999; Huk et al., 2001; Tolias et al., 2001a). Similar effects are reported in the orientation dimension for V1 (Fang et al., 2005) and the several stages along the ventral path (Fang et al., 2005; Boynton and Finney, 2003). Over time, the activity (metabolism) is reduced in an exponential manner (Tolias et al., 2001b). This may be seen as a fingerprint of an exhaustion or fatigue of the activated neuronal substrate.

Because of its parsimonious and appealing neurophysiological explanation of many psychophysical results, the usage-depended neuronal-fatigue model of adaptation was (see Mather, 1998, for review) and still is (van de Grind et al., 2004) debated. Accordingly, the only effect of adaptation is the gain-reduction of activated channels. That is, after adaptation to a certain feature, the cell is 'exhausted', such that a repeated stimulation with the same feature cannot be responded with the previous intensity (spike-rate). Given a population of neurons that represent the total feature-dimension (i.e. $0-359^{\circ}$ of motion), adaptation will reduce the 'gain' of the sub-population of cells that are sensitive to the adaptor. Presenting an ambigu- 
ous motion stimulus (e.g. 0\%-coherent RDP) thereafter would cause an unequal response profile of the population, such that the sub-population that was insensitive to the adaptor would have the relatively largest firing rate. This biased response of the population, in turn, can explain the characteristics of the MAE (van de Grind et al., 2004) and also most of the DAE . In the latter, the responses of the neurons to a truly directional signal biased in favor to the directions away from the adaptor's one. Much like the unadaptated response profile of a population looks gaussian, the post-adaptive gain-profile is an inverted gaussian centered on the adaptors direction. The largest discrepancy in the response and hence directional bias between sub-populations that code clockwise vs counter-clockwise from the test-stimulus is reached, when the test moves in the direction that maximizes the Fisher information of the gaussian profile. This is slightly larger than the half width of the population's tuning curve of $\sim 50^{\circ}$ and is in good agreement with the directional distance at which the DAE is largest (Levinson and Sekuler, 1976; Schrater and Simoncelli, 1998). Despite its ability to explain much of the psychophysical results and some largely undiscussed effects of 'inheritance' of adaptation effects across visual areas, other theories, apart from the gain change model, with a more sophisticated fundament are recently mostly tied to the adaptation process. According to the latter, the brain permanently tends towards an efficient evaluation of the environment.

'Efficient coding'-hypotheses of the brain have become of major interest during the last decade. In brief, the efficiency of the representation of a certain information content in coding system relies on both, the 'sources' amount of information and the 'drains' (coders) capacity to represent it. In the language of the neuronal tissue this means, that the strategy should be to represent information according to the statistical distribution of properties of the environment. Common entities of the visual environment should be in the center of the coding or spike-rate range of the neuron, rather than at either end. Spikes that code the same thing may be seen as redundant information carriers that are energy expensive (see Lennie, 2003, for review). According to Barlow (1961), the aim should be, to reduce the redundancy of the neuronal code through de-correlation of spiking activity to reach an 'optimal' compromise between energy investment and sensory representation. Mechanisms to reduce redundancy between two neurons that code very similar aspects of the visual world should either temporally decorrelate their activity by a down-regulation the respective spike-rates (de la Rocha et al., 2007) or/and separate the neurons sensory preferences. Both strategies would lead to a lower mean response to the 
given sensory input.

The orientation-sensitive areas of primates and cats (V1 and Area17, respectively) have long been the major sites to evaluate adaptational effects on the single neurons level. In summary, these neurons react to the presence of a preferred stimulus with strong response that exponentially decreases with longer stimulus durations Giaschi et al. (1993). This decrease is accompanied by an increased intracellular hyperpolarisation (Carandini and Ferster, 1997; Sanchez-Vives et al., 2000) and increased response latency (e.g. Saul, 1995). They show virtually no effect after 'null', i.e. orthogonal orientation, adaptation (Marlin et al., 1988; Giaschi et al., 1993). Together, these effects are the direct reflection of the contrast adaptation that shifts the contrast response function rightwards (Albrecht and Hamilton, 1982; Albrecht et al., 1984; Movshon and Lennie, 1979). Additionally, repulsive shifts of the orientation tuning curves, i.e. the selectivity, of striate neurons have been found (Nelson, 1991; Müller et al., 1999; Felsen et al., 2002; Dragoi et al., 2000, 2002; Crowder et al., 2005).

Unlike many psychophysicists investigated motion adaptation effects during the last two decades, the neuronal basis of this higher-vision phenomenon just recently reappeared in the scientific focus (see Kohn, 2007, for review). In the early days of neuronal recordings in area MT, Petersen et al. (1985) were the first who described the differences of 'preferred' vs 'null' adaptation on these neurons in anesthetized monkeys. Basically, these cells reduced their sensitivity to 'preferred' stimuli after 'preferred' adaptation for 20s and were not affected from null adaptation, similar to V1 neurons.

Van Wezel and Britten (2002) compared the adaptation effect of MT neurons in awake monkeys with the behavioral effect in humans during a motion detection task. Man and monkeys were adapted for 3 seconds with an either moving or stationary RDP. Thereafter, a test stimulus appeared on the monitor for one second. This could contain a variable percentage of dots that moved in either the adaptors or opposite direction (variable \%-coherence); the remaining dots moved in random directions, respectively. The human subjects had to judge the direction of the motion content of this high-contrast RDP; the monkey simply had to keep fixation on the screen. Interestingly, the amount of motion energy in the adaptors direction to compensate the MAE (point of subjective equality; PSE) in humans was fairly similar to the percentage coherent motion needed to evoke MT spike-rates usually evoked after contrast adaptation alone. Similar to Petersen et al. (1985), they did not find effects 
of null adaptation.

MT neurons receive their bottom-up input predominantly from area V1. Hence, the questions arose, how far the adaptation effects of MT are simply inherited from V1, and what would be MT's own contribution to motion adaptation (Kohn and Movshon, 2003). In the anesthetized monkey they recorded the responses of MT neurons to 'preferred' stimuli of varying contrast after 'preferred' adaptation at full contrast. However, the stimuli's diameter were chosen to be roughly half of the diameter of the RF of the current neuron. Such they were able to place either adaptor and test-stimulus at two non-overlapping positions inside the RF. Accordingly, they had 4 different spatial adaptor-test conditions and measured the effect of either combination on the cells contrast response function (CRF). In summary, they found that the rightward-shift of the CRF after adaptation became only visible, if the adaptor's and test's position overlapped (Kohn and Movshon, 2003). In turn, this means that adapting one 'subfield' of MT receptive fields does not cause contrast adaptation of the whole MT neuron and suggests that changes of contrast sensitivity occur prior to the spatial integration in MT. Contrast adaptation may hence be restricted to the early visual areas of V1 and lower.

In a second experiment they investigated the influence of adaptation on the feature-selectivity, i.e. direction-tuning, of MT neurons (Kohn and Movshon, 2004). The adaptation paradigm was similar to that described above, but now only one 'preferred' full contrast moving grating was used as adaptor. The test-stimuli were also full contrast and overlapped the adaptors position. However, they differed in the direction of motion - and orientation(!) - from trial to trial. Accordingly, Kohn and Movshon were able to reconstruct the cells tuning curves after adaptation. Besides the reduced sensitivity to the preferred direction by a factor of 0.7 , they also found a substantial bandwidth-change, i.e., a narrowing (factor: 0.54) of the tuning curve. They also looked for a change of the preferred direction of MT neurons after adaptation, as predicted by theory (Barlow, 1961; Barlow and Földiak, 1989). The adaptor's direction was therefore chosen to lay on the 'flank' of the tuning curve of the actual MT neuron. Hence, they adapted the cells with directions slightly different $\left(20^{\circ}-75^{\circ}\right)$ from their preferred direction. After adaptation, the tuning curve was again reconstructed. Interestingly, the cells changed their preferences (peak-response) attractively in favor to the adaptors direction of motion by $\sim 10^{\circ}$ on average. Shifting the adaptor further to directions that the cell was unresponsive to during the initial, unadapted measurements of the tuning curve had no effect on the tuning function. V1-cells, tested as control, did not exhibit such tuning curve 
shifts, which are therefore thought to emerge on the level of spatial integration in MT neurons. Together, these results are also in good agreement with the perceptual repulsion effects (Schrater and Simoncelli, 1998). Given a labeled-line principle, with each neuron 'voting' for its unadapted preferred direction, the attractive shift of its tuning should cause the 'new' preferred direction to be coded as the 'old' one. If the 'new' preferred direction would be presented, it would be 'seen' by hierarchically downstream neurons as the 'old' direction and may thus explain the repulsive DAE in addition to the effects of a gain-change-only.

However, the usage a grating with its orientation content as stimulus might introduce inherited adaptational influences of V1. Adapting and testing with a RDP would allow a better description of 'MT-only' effects, because V1 would be broardband stimulated and not with only one spatial frequency. In fact, Kohn and Movshon (2004) report in a subordinate clause, that they were not able to reproduce tuning curve shifts with RDP-adaptors.

As already mentioned in the previous section, also the effects of speed adaptation have been investigated (Krekelberg et al., 2006). The evaluation of the neurometric speed curves before and after adaptation shows, that an ideal observer relying on a single typical cell from these populations would report a decrease in perceived speed after adaptation. This is consistent with the behavioral report of humans and monkeys (Clifford and Wenderoth, 1999; Krekelberg et al., 2006; Thompson, 1981; Schrater and Simoncelli, 1998).

The experiments and data presented so far used always an adaptor with a specific feature, i.e. it was fixed at a point in the respective feature-space. The theory of adaptive mechanisms of the brain predict, however, also a change of the range of neuronal represented simulus values according to the recently present range of stimulus values (Barlow, 1961; Barlow and Földiak, 1989). Practically, that would mean for, e.g. a speed selective neuron to adapt such that the recently presented distribution of different speeds would define the range of its possible responses. These kind of experiments have up to now only been done in invertebrates, namely flies. Here, the H1-motion sensitive cells adapted to the variances of white-noise velocity signals presented to the fly (Fairhall et al., 2001). Such stimuli have a randomly switching direction (e.g. up and down) and also varying speeds. At a given stimulus interval of several (up to 40) seconds, the variance of speeds was constant and then switched to a higher or lower value and so on. Depending on the actual variance of the speeds, the same H1-neuron represented a speed range of about $\pm 50^{\circ} / \mathrm{sec}$. 
or $\pm 500^{\circ} / \mathrm{sec}$. with an equal range of possible response-rates, which could vary between $0.1 \mathrm{~Hz}$ and $\sim 200 \mathrm{~Hz}$. In other words, the bandwidth of the neurons coding capacity is adapted to the bandwidth of the world's speed distribution (see also Dean et al., 2005; Brenner et al., 2000).

In contrast to the smooth philosophical and theoretical explanations and expectations (Barlow, 1961; Barlow and Földiak, 1989) of adaptive mechanisms, the reality, revealed by measurements of neuronal activity in the adapting brain, is somewhat unwieldy. The usage of anesthetized and awake animals, recordings at different stages of the visual system, stimulation with different stimuli (e.g., gratings, random dot pattern, shapes, forms, colors, faces,...), stimulus configurations (whole-field, scenes, single stimuli) and the differences in the adaptation paradigms (milliseconds, seconds, minutes, minutes with top-up seconds), makes it impossible to extract the neuronal mechanism of visual adaptation. Depending on the constellation of the parameters above, there seem to be numerous explanations or combinations of different mechanisms that are plausible; or as Kohn (2007) recently formulated it: "...the biophysical underpinnings of neuronal [adaptation-]effects remain murky". Especially the oppositional effects of either repulsed V1 or attracted MT tuning curves after adaptation show, that a single prediction for such shifts (attraction only) does not hold the data.

Another open question concerns the 'de-correlation'-hypothesis of adaptation. Correlations of neuronal activity are amongst others measured by the power and frequency distribution of the LFP. However, in contrast to the investigations concerning the neuronal basis of attention (see section 1.4.2) effects of adaptation on the LFP are apparently missing.

Further investigations of the neuronal basis of adaptation, especially under natural viewing condition in awake, perceiving animals need to be done, to elucidate the true functional benefit of adaptive sensory processing under active, i.e. decision demanding, conditions.

\subsection{Visual attention}

\subsubsection{Attentional effects on visual perception}

If the visual environment changes, this may be a cue to decide also on a change of behavior. However, a more precise analysis of the changed or expected to change 
visual aspect can help us to make better, more 'valuable' decisions. One way to do this, is to change the line of sight and bring that visual patch into the scope of the fovea. The denser packing of the rods there allow first, a higher spatial resolution and, second, a better color discrimination compared to an extra-foveal analysis. Besides this so called overt attention mechanism, another form of attention exists: covert attention.

Covert attention or in short 'attention', is a mechanism that has in principle similar effects on perception as overt attention, but differs from the latter such that it is not accompanied by a gaze shift. Instead, a virtual attentional focus or 'spotlight' is thought to shift and elucidate otherwise neglected parts of the visual field (Posner et al., 1980). Spatially directed attention reduces detection thresholds by an increase the of the contrast sensitivity (Lu and Dosher, 1998; Carrasco et al., 2000; Cameron et al., 2002), i.e. better detectability of a faint stimulus, and increases the discriminability of the attended stimulus' properties. Attentional performance is also manifest in reduced reaction-times (e.g. Treisman and Gelade, 1980)) during search, increased hyperacuity and spatial resolution (Yeshurun and Carrasco, 1998). Finally, it also alters the apparent size of stimuli, which appear to be larger (Carrasco et al., 2004; Anton-Erxleben et al., 2007).

Three 'classes' of visual attention can be distinguished. First, the abovementioned spatial attention, second, the feature-based and, third, object-based attention. During feature-based attention the representation of the attended feature (e.g., the color red) is enhanced - non-spatially - throughout the visual field (Rossi and Paradiso, 1995; Saenz et al., 2002; Maunsell and Treue, 2006, for review). This helps in visual search, where a target item (featured object) might be found faster and more reliable (see Treue and Martínez-Trujillo, 2007, for example). Attention, when directed towards an object, can enhance the representation to all of its features, (colors, orientations,...) and may even elucidate parts of the object that reemerge behind an occluding cover-(object) (Duncan, 1984; Valdes-Sosa et al., 1998, 2000; Blaser et al., 2000).

However, there exist striking differences in the mode of action of attention, that depend on the way how it is guided and evoked. The appearance of a salient stimulus somewhere in the visual field acts as an exogenous cue and automatically captures attention, which in turn boosts the computational power at that location (Itti and Koch, 2001). This mechanism cannot be suppressed by the subject and is thought to usually precede the overt attentional gaze shift towards objects of - possible interest (Theeuwes, 1991; Itti and Koch, 2001). However, the exogenously guided 
attentional focus is transient. This was shown by Posner and Cohen (1984) in a classical human psychophysics experiment: a spatial location on the computer-screen was cued with $80 \%$ validity and the subject had to detect a successively presented stimulus at either the cued or another screen-position. The reaction-time was lower for a stimulus at the cued position compared to the un-cued location, but only for about the first $100 \mathrm{~ms}$ after the cue disappeared. After about $300 \mathrm{~ms}$ the cued location was not better represented any more and thereafter, stimulus appearances at the uncued position caused faster reaction-times compared to a control without any cue. After about $1000 \mathrm{~ms}$ cue-target onset asynchrony, the cue had no influence on either performance any more. This phenomenon was termed Inhibition of Return (IOR, see Klein (2000) for review). I.e, spatial attention is only transiently at a certain position. Endogenously cued ('covert') attentional focussing that bases on the knowledge of a rule, however, does not suffer from IOR.

In addition, the measurable effect of attention on perception is not only the enhanced visibility of otherwise neglected stimuli, but in contrast the active masking of unattended - despite salient - stimuli or features in favor to the attended ones. Even putative 'obvious' changes between two otherwise equal visual scenes are neglected if they are viewed in temporal succession, simply interrupted by a short $(\geq 80 \mathrm{~ms})$ blank stimulus. Rensink et al. (1997) termed this phenomenon 'change blindness'. In a rather spectacular experiment Simons and Chabris (1999) even showed that during the inspection of natural movie, when attention was instructionally directed towards a white-dressed basketball-team among a black-dressed team, many subjects did not recognize the rather absurd incident of a person, disguised as a black gorilla, walking through the scene. Their findings further suggest that attention is an important mechanism that allows the perception of aspects of the visual world at all (Simons, 2000; Simons and Rensink, 2005); hence Simons terms this perceptual blindness to unattended stimuli 'inattentional blindness' and take the phenomenon as evidence for a 'sparse representation' of visual informtion.

On a final note, attention increases the effects of visual motion adaptation (Chaudhuri, 1990; Alais and Blake, 1999; Rezec et al., 2004) such that MAEs and DAEs are enhanced. This shows that attention not only manipulates the 'recent' visual input but also affects perceptual performances in the future. In turn, the timecourse and effects of adaptation in the neuronal substrate during active vision differ from that of passive bottom-up stimulation of anesthetized monkeys or cats, as used in many physiology studies. 
Taken together, the psychophysical investigations lead to the question about what neuronal (-network) mechanisms are responsible for this plastic, variable, and goal-driven evaluation of the visual world .

\subsubsection{Neuronal correlates of visual attention}

Correlates of visual attention with the brains' metabolism and single cells activity have been confirmed in humans and monkeys. During the last decade the fMRI technique revealed that besides the higher visual areas (Kastner et al., 1998; Tootell et al., 1998; Saenz et al., 2002; Pessoa et al., 2003; Yantis and Serences, 2003; Murray and Wojciulik, 2004; Silver et al., 2007; Serences and Boynton, 2007) also V1 (Tootell et al., 1998; Silver et al., 2007) and even the LGN (O'Connor et al., 2002) are attentional modulated.

But how does this modulation look like and how are the mechanism implemented? Single neuron investigations have already revealed attentional modulation in different higher visual areas of macaque monkeys since the mid 80's of the 20th century (Moran and Desimone, 1985; Haenny and Schiller, 1988; Haenny et al., 1988; Spitzer et al., 1988). These and following studies reported different changes of the RF properties under attentional condition compared to passive viewing. During the first study by Moran and Desimone (1985), cells of the ventral pathways area V4 were recorded. Meanwhile, two stimuli were placed in the neurons RF: one matched the cells preferred orientation and color, the other was of an orthogonal - non-preferred - orientation and color. The monkey performed an identity test on either of these stimuli (the attended one) and received a juice reward for a correct 'answer'. Moran \& Desimone found, that the neuronal response to the pair of stimuli was stronger, when the preferred stimulus was in the attentional focus compared to the condition when the non-preferred stimulus was attended. Since then, such finding that the attended stimulus inside the RF critically determines the response characteristic of the neuron has been corroborated in many neurophysiological studies of the ventral (Connor et al., 1996; Chelazzi et al., 1993, 2001; Motter, 1993; Sheinberg and Logothetis, 2001; Luck et al., 1997; Reynolds et al., 1999; Reynolds and Desimone, 2003) and dorsal (Treue and Maunsell, 1996; Treue and MartínezTrujillo, 1999; Martínez-Trujillo and Treue, 2002; Gottlieb et al., 1998; Bisley and Goldberg, 2003; Wannig et al., 2007) visual pathway.

But also V1 neurons already show attentional modulations of their responses (Motter, 1993; Roelfsema et al., 1998, e.g.). However, because the RFs of V1 neu- 
rons are rather small, only one stimulus was present inside the RF in these studies. This shows that already on the early stages of the visual system attention can selectively filter out aspects of the visual world.

Additionally, the existence of different 'kinds' of attention on the level of psychophysics (spatial, feature-based, object-based) was also proved on the neuronal level. Such, object-based effects have been found in V1 and V4 (e.g. Roelfsema et al., 1998; Kreiman et al., 2006); feature-based effects are present in V4 and MT (e.g Motter, 1994; Treue and Martínez-Trujillo, 1999; McAdams and Maunsell, 2000; Martínez-Trujillo and Treue, 2004; Maunsell and Treue, 2006, for review).

Together, psychophysics and electrophysiology revealed, that the representation of an attended stimulus is favored at the expense of that of other stimuli. Based on the psychologists view Desimone and Duncan (1995) derived their so-called biased competition hypothesis of attention. They and others (Reynolds and Desimone, 1999; Pessoa et al., 2003) stated, that 'objects' of the visual world per se are 'competing' for neuronal resources and thus perception by means of mutual interactions in the visual cortex. This competition could be won, either because the strong bottom-up salience (i.e., feature contrast to the background or/and other 'distracting/competing' stimuli) or because the voluntary top-down pushing mechanism causes an outstanding response in the sensory neurons. Although it is known, that spatially neighboring visual sub-fields can mutually interact with the 'classical RF' (Hubel and Wiesel, 1968) (e.g. through lateral inhibition as in center-surround mechanisms of visual neurons), the nature of the competition is typically left unspecified.

However, a more mechanistic and parsimonious explanation - implicitly part of the biased competition hypothesis - is, that the neuronal response is 'simply' modulated in a gain-change manner such that the representation of a behaviorally relevant stimulus-feature and/or -position is multiplicatory boosted in all relevant visual areas. Simultaneously, response normalization (Carandini et al., 1997; Simoncelli and Heeger, 1998; Britten and Heuer, 1999; Heuer and Britten, 2002) scales the responses of all neurons of a visual area (see section 1.1.1). The attentional benefit is therefore not only measurable in the absolute response increase but also in the larger relative differences of spiking neurons, i.e. response contrast. This becomes evident in the study by Martínez-Trujillo and Treue (2004) on feature-based attention in MT. When an attended stimulus outside the RF matched the preferred direction of the recorded neuron, its responses to a preferred stimulus inside its RF were increased. However, when the stimulus inside the RF moved in the null di- 
rection and attention was still on the preferred direction outside the RF, the neurons responses were even lower, than without attention on either moving stimulus. Although these results were gained in the motion-dimension, they are impressively in accordance with the perceptual suppression of unattended features as seen in the above mentioned 'inattentional blindness'-experiments of Simons and Chabris (1999). In MT, the angular feature-similarity between the attended feature and the preferred direction of the neuron is positively correlated to the factor of the multiplicative attentional gain modulation. Angular distances larger than $\sim 90^{\circ}$ result in a gain factor lower than 1 .

Physiological evidences for such a multiplicative gain-change mechanism have been independently described for spatial attention tasks in motion sensitive MT neurons (Treue and Martínez-Trujillo, 1999) and orientation selective V4 and V1 neurons (McAdams and Maunsell, 1999). During the experiments of Treue and Martínez-Trujillo (1999), the rhesus monkeys either had to detect a speed-change in one of two simultaneously presented random dot pattern that were moving behind two spatially separated virtual apertures. Either the stimulus inside the RF of the MT neuron or outside the RF could be in the attentional spotlight. Distracting speed-changes in the unattended movie had to be neglected. The mean attentional modulation of MT neurons was consistently $10 \%$ across all directions. In the study from McAdams and Maunsell (1999), the monkeys had to solve a delayed matchto-sample task: two stimuli (one oriented grating and one color patch) were shown simultaneously for $500 \mathrm{~ms}$ on a computer-screen. After a brief (500ms) delay with only a fixation spot visible, the two stimuli reappeared. However, either the color or the orientation of the grating could differ from the initial presentation. The monkey had to report such a change - only - at the beforehand cued spatial location by retaining an otherwise given response. Such, McAdams and Maunsell collected data for a complete orientation tuning curve with and without attention. They found that orientation selective V4 cells responses are in average upregulated by $22 \%$. During the same experiment they also recorded the activity from V1-cells. Theses cells were only modulated by about $8 \%$. Together with the results of other studies that investigated even higher visual areas (MST, VIP, 7a: Treue and Maunsell (1999); Cook and Maunsell (2002)) and found modulations up to $\sim 80 \%(!)$, this is in accordance with the idea, that attentional modulation 'acts' independently (Maunsell and Cook, 2002) on each level of the 'cortical hierarchy' (Felleman and Van Essen, 1991). I.e., the upregulated response of one area is further boosted in the following processing stage. 
A second outcome of the two studies, that is directly derived from the fact of multiplicative scaling, is, that the feature selectivity (orientation, direction of motion) of the neurons did not change under attention (contra Spitzer et al. (1988)). I.e., the width of the tuning curves stayed the same with and without attention. This is an important finding, because also the spatial selectivity of MT-neurons does not substantially vary with the spatial focus of attention as we can show in one paper of this thesis (chapter 4). I will put further emphasis on the tuning aspects below.

How is the multiplicative gain change implemented in the brain? A 'natural' factor that determines neuronal response strength of visual is the contrast (Albrecht and Hamilton, 1982) and has similar effects as attention on V4 and MT neurons (McAdams and Maunsell, 1999; Treue and Martínez-Trujillo, 1999). For example, increasing the contrast of a sinusoidal grating typically increases the response of a neuron to all orientations proportionately, such that a tuning curve constructed from responses to high-contrast stimuli is a multiplicatively scaled version of one made using lower contrast stimuli (Skottun et al., 1987). The neuronal response is a monotonic function of the logarithm of stimulus contrast. Typically the contrast response function (CRF) has a sigmoidal shape. Very low contrasts elicit no response and high contrasts yield the cells to reach an upper response-limit, that cannot be further increased by increasing the contrast. In between these two fixpoints, the response curve has the mentioned, non-linear form (e.g., see Albrecht and Hamilton (1982) and Thiele et al. (2000)) that can be a achieved through a multiplicative mechanism. The perceptual and behavioral similarities between contrast enhancement and attentional 'spotlighting' (i.e., lowering of the perceptual threshold) led to the questions about similarities of both mechanisms in the neural structure. Multiplicative modulation of visual stimuli by attention that shift the inclination point of the sigmoidal CRF (i.e., the contrast, at which the cell reaches $50 \%$ of its maximum firing rate; 'C50') leftwards to lower contrast could act in two ways. First, the responses of the neuron are up-regulated by the same factor, irrespective of the stimulus' contrast. This is known as 'response-gain-model' of attention. An important result of such a mechanism would be, that also very high contrasts, that usually saturate the cells responses would drive the cells response beyond this boundary. The second mechanism assumes a more or less fixed maximum firing rate of the neurons. Such, the C50-shift would be evoked by a pure horizontal shift of the CRF. This means that higher contrast stimuli, that would evoke strong response without attention could drive the cells into saturation with attention. On the other hand, contrast that would 
not evoke a neural response in the pure sensory conditions elicit some spike production with attention. This means, the multiplicative factor would in the end not be fixed across all contrasts but vary bell-shaped across contrast and have some peak at intermediate contrasts.

Reynolds et al. (2000) and Martínez-Trujillo and Treue (2002) addressed this question in physiologial studies in area V4 and MT of rhesus monkeys, respectively, where they varied the contrast of the stimuli inside the spatial attentional focus. Although the stimulus configuration and the temporal succession differed between the studies, both found - directly or indirectly - the strongest attentional effects on low and intermediate contrasts, with no or only small effects on high contrasts. Despite there is some kind of 'response-gain-effect', the results are better explained by the 'contrast-gain-model'. This might also explain the differences in the attentional effect size across studies: McAdams and Maunsell (1999) used intermediate contrast stimuli and had attentional effects of $22 \%$ whereas Treue and Martínez-Trujillo (1999) used high contrast stimuli and had only $10 \%$ effect. However, Williford and Maunsell (2006) recently reported that the response change of their tested V4 neurons under attention can be better explained by a 'response-gain-model'. They even accentuate, that a so-called 'activity-gain-model', fits best their measurements. This is basically an additive effect on the responses, such that even lowest contrasts of the CRF are modulated, which would be equivalent to an increase of the neurons baseline (without stimulus) firing rate. The question remains, how far the stimulation protocol of briefly (94ms) flashed sinusoidal gratings and the short pause $(94 \mathrm{~ms})$ between the stimuli affected their results. First, the time between two stimuli is comparably short, which introduces an uncertainty about which stimulus evoked a certain spike and, second, they only measured the transient response of the neuron to the stimulus. This time-period has different 'rules' than the sustained response period to a longer stimulus (see chapter 3).

A caveat of multiplicative modulation models of attention are some discrepancies to fMRI studies. Accordingly, attentional effects should be week or absent for very low contrasts (as confirmed by single-unit recordings). However, at least three fMRI studies showed a substantial increase of the BOLD signal in attention tasks during the absence of a stimulus, which is by definition a zero contrast (Kastner et al., 1999; Silver et al., 2007; Serences and Boynton, 2007). Buracas and Boynton (2007) recently reported, that according to their fMRI data they would favored additive effects of attention in human $\mathrm{V} 1, \mathrm{~V} 2, \mathrm{~V} 3$ and MT+. It remains to be clarified, if 
an increase of dendritic activity, that is not accompanied by an increase in the spike rate can account for these results.

The basic ideas of efficient neural coding should hold true also in the case of attention. As much as during adaptation the correlated activity of neurons should decrease, it should increase with attention. This can, as explained above, be realized by a firing-rate increase (de la Rocha et al., 2007) and, in theory, also by a change of some neurons' preferences and selectivity, e.g. preferred coded feature and width of their tuning-curve. Attention could shift the tuning preferences of units that 'almost' represent the attended feature (e.g., direction of motion) towards this feature. More similarly tuned neurons would deliver the fundament for a fine-grained resolution and thus higher acuity with attention than without it. Again, despite intensive search, such preference-change has not been found (Vogels and Orban, 1994; McAdams and Maunsell, 1999; Treue and Martínez-Trujillo, 1999). However, Connor et al. (1996, 1997) and we (Womelsdorf et al., 2006a, see chapter 4) found such a shift of preferred coded spatial position. I.e, the point of maximum spatial sensitivity of V4 or MT neurons RF, respectively, shifts towards the spatial focus of attention. Although there are several possible mechanisms that create such a spatial shift of the RF (Compte and Wang, 2006), a very simple model that acts also in a multiplicative manner is a feedforward-model. Here, the (retinotopic) neurons layer upstream (e.g. V1) to the higher visual area (e.g. MT) neuron under observation are selectively enhanced by spatial attention. Because the information of many V1-neurons converges on one MT neuron, the input to this neuron is spatially biased by attention (McAdams and Maunsell, 1999).

Higher correlation of neuronal activity is also known in the context of synchronous discharge of neurons. This can be measured either as temporally correlated spike occurrences or in by the amplitude of certain frequencies in the low frequency range $(1-150 \mathrm{~Hz})$ of the extracellular electric field potential (local field potentials; LFP). Additionally, the correlation of spikes with the phase of the LFP is another measure of correlated activities between neuron-groups. The LFP is thought to have its origin in the local dendritic activity, rather than in the summed spiking activity (Logothetis, 2003). Temporally correlated activity - synchrony or coherence of neurons in one area could increase their impact in the sense of evoked activities in post-synaptic neurons - especially if the input converges. Recently, this kind of 'feed-forward boosting' and guidance of sensory information is also investigated in the light of attention. 
For V4 neurons, Fries et al. (2001) reported that the relationship of individual spikes and frequencies of the LFP (spike-field-coherence) changes with attention. While the stimulus in the cells RF was unattended, the evoked spikes were almost equally strong coupled to LFPs in the $\alpha$-range $(8-12 \mathrm{~Hz})$ and in the $\gamma$-range (30$80 \mathrm{~Hz}$ ). However, when attention was directed towards the stimulus inside the RF, $\alpha$-power was reduced and $\gamma$-power increased accordingly by about $10 \%$. Hence, it is not synchrony or coherence per se that is increased. Instead, frequency ranges are affected differently. This task related variable $\gamma$-power is proposed as crucial mechanism in visual perception (see Womelsdorf and Fries, 2007, for review).

The critical experiment that would underline the importance of neuronal coherence would have to show, that behavioral attentional effects disappear or are reduced when the cells loose synchronous activities, despite firing with the same rate. Womelsdorf et al. (2006b) addressed exactly this question. They correlated the reaction-times of monkeys in a visual change-detection task to the neuronal coherence between spikes and the LFP as well as to the LFP power. In brief, when they sorted the trials of each experimental session according to the monkeys reactiontimes and then built groups out of the fast- $(\sim 350 \mathrm{~ms})$ and slow-reaction-time quartile $(\sim 490 \mathrm{~ms})$ of trials, the spike rates of the sensory V4 neurons did not differ between the groups around the time, the monkey responded. However, when the stimulus inside the RF was attended, fast reaction times were associated with an increased gamma-band synchronization before and after the response. Conversely, the slow reaction-time-trials had a lower gamma-band synchronization. These relationships even inversed when the attended stimulus was outside the RF. This is explained in the following way: High gamma-band synchronization during slow reaction-time trials with attention outside shows, that the distractor (inside the RF) has actually a stronger impact on the perception and, in turn, reduces the speed of information-transmission at the attended location. Together with the results from other recent studies (Taylor et al., 2005; Buschman and Miller, 2007) this indicates a functional role of attentional neuronal synchronization in the gamma-range.

As much as the attentional impact on sensory neurons may vary between trials, it can be dynamically regulated within trials. As I already mentioned, deciding is the expression of making assumptions about the future, given the recent state of the 'self', the environment and the history of decisions. In usual neuro-behavioral experiments, the subjects have to react to a critical event in the stimulation, that appears some times after the start of a trial. However, the instantaneous probability of 
such an event, namely the likelihood that it will appear 'now', given that it did not appear yet, changes across the trial of classical attentional experiments. This conditional probability is called the hazard function. Again, according to the efficient coding hypothesis, it would be advantageous to attend - and invest extra-spikes based on the hazard function. Conversely, it would be a waste of energy to spend spikes for the representation of stimulus that is currently not expected to change. In two studies, either Ghose and Maunsell (2002) (in V4) as well as Janssen and Shadlen (2005) (in LIP; dorsal path) found such a variable allocation of attentional resources according to the instantaneous probability of the critical event. In the V4study the monkeys had to detect a change in a spatially attend stimulus, while during the LIP-study, the monkey had to make a saccade to an appearing target after a variable delay. Two different hazard rates were tested en-block, respectively, such that the monkeys could know the actual underlying hazard function from experience. Moreover, Janssen and Shadlen (2005) reported a coupling of the reaction-time to the hazard rate: when the hazard rate was low, i.e. a critical event was unlikely, the reaction times were high and vice versa.

The control of voluntary attentional modulation is thought to have its origin in parts the frontal lobe, namely the frontal eye field (FEF). Electrical stimulation of such FEF neurons cause saccadic eye movements Hanes et al. (1995) and different regions of FEF code for different trajectories of the gaze shift. In his 'premotor theory of attention' Rizzolatti (1983) hypothesized that spatial attention is a mechanism to 'pre-evaluate' parts of the visual field that are upon to be in the retinas focus after the fortcoming saccade. In a noteworthy experiment (Moore and Armstrong, 2003; Moore and Fallah, 2004) stimulated FEF neurons of rhesus monkeys while these fixated a spot on the monitor. Such a suprathreshold stimulation caused the monkey to change his gaze towards another position on the screen (its gaze field). Moore and colleagues now searched for FEF positions that, if stimulated, would cause a gaze shift inside the RF of a simultaneously recorded V4 neuron. After they have found such an FEF position, they electrically stimulated it with a subthreshold current. Interestingly, FEF-stimulation caused the V4 neuron to respond stronger to a visual stimulus inside its RF as without electrical stimulation. These findings show that electrical stimulation of frontal areas biases the responses of neurons in visual areas in a similar manner to that observed during voluntary shifts of attention and support Rizzolattis ideas. To term this with the strikingly formulation of Treue and Martínez-Trujillo (2003), attending means: "Moving the Mind's Eye Before the Head's Eye". 


\section{Chapter 2}

\section{Brief motion adaptation causes repulsive aftereffects}

Visual adaptation has been a powerful tool for the psychophysical investigation of human vision. So far most visual adaptation studies have used long durations (in the rage of tens of seconds to minutes) of adaptation. However, during everyday vision the retinal image is changing at a much faster pace, due to eye movements and relative motion of the environment. If substantial adaptation effects would be restricted to the long exposure durations of the typical laboratory experiment the proposed benefits of adaptation (energy- and information-efficient information coding) would not play a role in real life.

Here we show that visual motion adaptation causes large repulsive direction aftereffects (DAE) after as little as 2 seconds of adaptation. In addition, we show that these findings can be easily accounted for by a simple neuronal gain-change-model of adaptation.

This manuscript makes an important contribution to the ongoing debate on the role of adaptation in everyday vision. Furthermore, the results will encourage investigators to elucidate the neuronal mechanisms of adaptation in the awake and behaving animal. 



\title{
Brief motion adaptation causes repulsive aftereffects
}

(prepared for submission)

\author{
Florian Pieper ${ }^{1}$ \& Stefan Treue ${ }^{1}$ \\ ${ }^{1}$ Cognitive Neurosciences Laboratory, German Primate Center, Kellnerweg 4, 37077 Goettingen, Germany
}

\begin{abstract}
Prolonged sensory stimulation causes adaptive changes in the processing of sensory information. For example in the motion aftereffect (MAE), exposure to continuous unidirectional motion causes a subsequently presented static test stimulus to be perceived to move in the direction opposite to the adapter. For moving test stimuli the direction aftereffect (DAE) creates a systematic perceptual overestimation of small adapter-test direction differences. Such phenomena have traditionally been investigated with adaptation durations of tens of seconds to minutes. Here we show that substantial adaptation effects in the human visual motion system occur with very short adaptation durations of as low as 2 seconds. Thus adaptation affects sensory processing even in viewing conditions more in line with the dynamics of everyday vision.
\end{abstract}

Keywords: DAE, direction aftereffect, MAE, motion aftereffect, psychophysics

Corresponding author:

Florian Pieper

German Primate Center

Cognitive Neurosciences Laboratory

Kellnerweg 4

37077 Goettingen

GERMANY

email: fpieper@gwdg.de

phone:++49(0)551-3851-345

fax: $\quad++49(0) 551-3851-452$

\section{Introduction}

A long history of psychophysical studies (see Clifford \& Rhodes, 2005 for reviews) documents that prolonged exposure to a sensory stimulus induces adaptation. This transiently reduced sensitivity to the stimulus' features ${ }^{1}$ (e.g. the orientation of a line or the direction and speed of visual motion) (Gibson, 1937; Levinson \& Sekuler, 1976; Thompson, 1981) represents a history-depended coding of the world as the nervous system reversibly changes its response to a sensory input depending on the statistics of the preceding input on a time-scale of seconds to minutes. The apparent disadvantage of a reduced sensitivity for the adapted feature is offset by an increased coding efficiency since the nervous system invests less energy in spikes (Lennie, 2003) that represent features of the world that are continuously present and thus contain little new information. Instead, an adapted sensory system is particularly sensitive to any deviations from the prevalent input pattern.

Adaptation also increases coding efficiency whenever a stimulus activates several neurons. Neurons that represent the same aspect of the environment (such as a particular color, orientation, direction of motion, pitch or face) produce highly correlated spikes. To reduce this redundancy such groups of neurons, should reduce their correlated activity (Barlow \& Földiak, 1989) by either changing their stimulus preferences as expressed in their tuning curves, i.e. the response distribution along the encoded stimulus dimension and/or reduce their firing-rate. With such a gain reduction in the face of constant sensory input spikes from the independent neurons are more likely to be temporally dispersed and thus will exhibit a lower temporal covariance (Müller, 1999). In toto adaptation will therefore reduce the allocation of encoding and

\footnotetext{
1 Throughout this manuscript we use the term 'feature' to denote a particular value of a stimulus dimension. For example, the colors red and green represent
} two different features. 
processing resources in regions of feature space occupied by unchanging aspects of the sensory input.

A multitude of physiological studies agree with these predictions, e.g. adaptation has been shown to cause shifts of tuning curves for orientation-selective (Dragoi et al., 2000; Müller et al., 1999) and directionselective neurons (Kohn \& Movshon, 2004) as well as increased hyperpolarization (Carandini \& Ferster, 1997) and response decreases (Movshon \& Lennie, 1979; Dragoi et al., 2000; Giaschi et al. 1993) in V1 or MT neurons (Petersen et al., 1985; Kohn \& Movshon, 2004; Krekelberg et al., 2006) responsive to the adapting stimulus.

Since the distribution of activity across populations of neurons conveys important information about the sensory environment, the internal changes to sensory processing caused by adaptation have perceptual consequences. The waterfall-illusion (Wohlgemut, 1911) or motion aftereffect (MAE) (see Mather et al., 1998 for review) is a prominent example of the perceptual consequences of adaptation. After prolonged (tens of seconds to minutes) inspection of a pattern moving in a single direction, a static or noise pattern appears to move in the opposite direction. Further effects of visual motion adaptation are an increase of the detection threshold for the adapted direction (Hol \& Treue, 2001) and an increase of the discrimination threshold for motion directions deviated approx. $40-60^{\circ}$ from the adapted direction (Regan, 1985; Hol \& Treue, 2001). Furthermore a difference between an adapter's direction and that of a successively presented brief test stimulus leads to a direction aftereffect (DAE), i.e. a perceptual motion repulsion that causes an overestimation of the angle between adapter and test (Levinson \& Sekuler, 1976; Schrater \& Simoncelli, 1998; Curran et al., 2006).

Traditionally investigations of perceptual perturbations after adaptation employ long adaptation durations of tens of seconds to minutes followed by a brief test period and top-up adaptation of a few seconds with the same adapter to maintain the adapted state. However, as eye positions during free viewing usually shift up to several times a second (Land, 2004) and periods of maintained fixation rarely last more than a few seconds, the observed perceptual aftereffects may not be of much relevance in natural viewing and decision-making. If the visual neuronal apparatus is designed to optimize its representation of the sensory input and thus improve perception and save energy, this should happen on a much shorter time-scale than usually tested.

Only a small number of psychophysical studies have investigated such short-term adaptation. Suzuki (2001) showed that an adaptation to a static stimulus presented for 2.6 seconds and less can evoke shape perception aftereffects. Recently, Krekelberg and Albright (2006) showed, that after 2 seconds of motion adaptation speed perception is altered and provided some evidence for functional correlates from neural recordings in monkey area MT.

Here we report the results of brief adaptation on the perception of the direction of visual motion. Human subjects had to judge the direction of a moving random dot pattern (RDP), after a brief adaptation with an RDP moving in a different direction. We found the perceptual motion repulsion described above, i.e. a direction aftereffect, already after only 2 seconds of adaptation indicating that adaptive processes occur on a timescale useful for natural viewing conditions.

\section{Methods}

Subjects: Ten naive subjects (6 females, 4 males) participated in the experiments. All except one highschool student were college students at an age between 15 and 33 (mean: 23.5; std.: 4.6). The volunteers had normal or corrected-to-normal vision and gave their written consent to participate in the study. Prior to the experiment all subjects were trained on an extrafoveal direction discrimination task until they were able to discriminate deviations from vertical motion of less than $3^{\circ}$.

Stimuli: The experiment was performed using a custom written Apple Macintosh software for stimulus presentation and behavioral control. The stimuli were displayed on a black $(0.1 \mathrm{~cd} / \mathrm{m} 2)$ background on a CRT Monitor (LaCie, 'elec-tron22bluelV') at a resolution of $38 \mathrm{pixel} / \mathrm{deg}$ and a refresh rate of $85 \mathrm{~Hz}$. Subjects sat in a dimly illuminated test cabin with the head on a chin rest to maintain a viewing distance of $85 \mathrm{~cm}$. All stimuli were random dot patterns (RDP) moving within a virtual stationary circle with a density of 12 dots/deg2 where each dot had a size of $0.08^{\circ} * 0.08^{\circ}$ and moved at a speed of $5 \mathrm{deg} / \mathrm{sec}$. In a given session stimuli were centered $8^{\circ}$ left or right of a gray fixation point, respectively. The dots of the adapter stimulus were displayed at full luminance $(68 \mathrm{~cd} / \mathrm{m} 2)$ whereas the test stimulus' dots were dimmer $(34 \mathrm{~cd} / \mathrm{m} 2)$. 


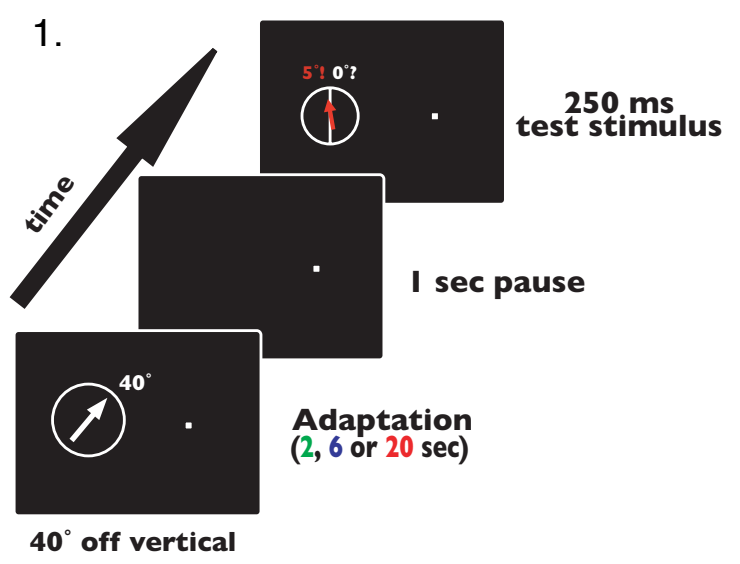

Figure 1: Subjects had to fixate and adapt to random dot pattern (RDP) moving at $100 \%$-coherence $40^{\circ}$ off vertical $\left(0^{\circ} \%\right.$ $\left.180^{\circ}\right)$. From trial to trial one of four adaptor directions $\left(40^{\circ}\right.$, $140^{\circ}, 220^{\circ}$ or $320^{\circ}$ ) were shown. After the adaptation period, which could last between 2 seconds and 20 seconds, and an ISI (Pause) of 1 second, they had to judge the direction of motion of the following test-stimulus as either being tilted clockwise or counterclockwise from the internal representation of the vertical. $40^{\circ}$ and $320^{\circ}$ adaptors were tested with a $0^{\circ} \pm 18^{\circ}$ vertical test stimulus while $140^{\circ}$ and $220^{\circ}$ adaptors were tested on $180^{\circ} \pm 18^{\circ}$ directions. In the $140^{\circ}$ off-vertical condition the adaptors were physically the same as in the 6 $\sec 40^{\circ}$ off-vertical adaptation period, but now the following tests were chosen from the distribution of the opposite 'verticals'. The example shows the principle effect of motion repulsion: After $40^{\circ}$ clockwise adaptation a stimulus moving exactly to $0^{\circ}$ is perceived to move at, e.g., $5^{\circ}$ counterclockwise. Therefore an about $5^{\circ}$ clockwise moving stimulus would be perceived to move upwards $\left(0^{\circ}\right)$. During the baseline measurements there was no adaptor present, during the random motion adaptation period, 6 seconds of a $0 \%$ coherence ('random noise') RDP were shown.

To compensate for small, involuntary eye movements and ensure that the test stimuli would stimulate a previously adapted part of the visual field, the adapter had a larger diameter $\left(8^{\circ}\right)$ than the test stimulus $\left(5^{\circ}\right)$.

Procedures: In a two-alternative forced choice (2-AFC) direction discrimination task subjects were first shown an adapting stimulus and then had to report whether the direction of a successively presented test pattern deviated clockwise or counterclockwise from vertical. For each subject the experiment consisted of 12 blocks (six adaptation conditions* two visual hemifields) of 100 trials each (Figure 1). Subjects were instructed to maintain fixation on a central fixation point throughout each trial. They initiated each trial by pressing a key on a computer keyboard. 200ms after trial start the adaptor appeared. After its offset and a stimulus-free interval of 1 second the test stimulus was presented for $250 \mathrm{~ms}$ at the adaptor's position. Test stimuli moved either globally 'upwards' or 'downwards' and were chosen from a range $-18^{\circ}$ to $+18^{\circ}$ from the vertical in steps of $2^{\circ}$. The subject reported whether the test direction deviated clockwise or counterclockwise from vertical by pressing one of two keys on the keyboard. After approx. 1-2 seconds subjects initiated the next trial.

The following six adaptation conditions where tested: $100 \%$-coherent motion adaptors of 2, 6 and 20 seconds duration moving at $40^{\circ}$ relative to the vertical (up or down) as well as 6 seconds $100 \%$-coherent motion RDP at an angle of $140^{\circ}$ relative to the vertical. Baseline performance was measured without adaptors and effects of contrast- and local motion-adaptation was determined using 6 seconds of $0 \%$-coherent motion RDP adaptors.

To avoid cumulative adaptation to a certain direction across several trials in an experimental block, the adaptor direction was randomly selected for each trial out of the four directions used. The $40^{\circ}$ deviation was chosen, be-cause it created large repulsive effects in studies using long duration adapters (Levinson \& Sekuler, 1976; Hol \& Treue, 2001; Schrater \& Simoncelli, 1998) while the strongest attractive effects have been reported with a $140^{\circ}$ deviation between adapter and direction (Schrater \& Si-moncelli, 1998). In all 40 trials upward moving adapters were always combined with upward moving test stimuli and downward adapters were paired with downward test stimuli; and vice versa for $140^{\circ}$-trials.

For choosing each trials' test direction, a weighted up-down staircase procedure (Kaernbach, 1991) was used. This ensured that the subjects' choices of clockwise vs. counter- clockwise deviations were approximately equated and con-centrated the test directions near the subjects 'point-of-perceivedvertical' (PPV).

Data analysis: We obtained the response distributions as a function of the test direction and fitted them with a logistic model

$$
P(x)=1 / 1+\exp (-b(x-a))
$$

where $x$ is the direction of motion of the test stimulus relative to the adaptors direction, $P(x)$ is the corresponding hitrate, a corresponds to the inclination point of the function, corresponding to the subject's perceived vertical and $b$ is used to compute the discrimination threshold defined by

$$
\sigma_{\text {exp }}=x_{p=.84}-x_{p=.50}=(1 / b) \ln (21 / 4)
$$


2.

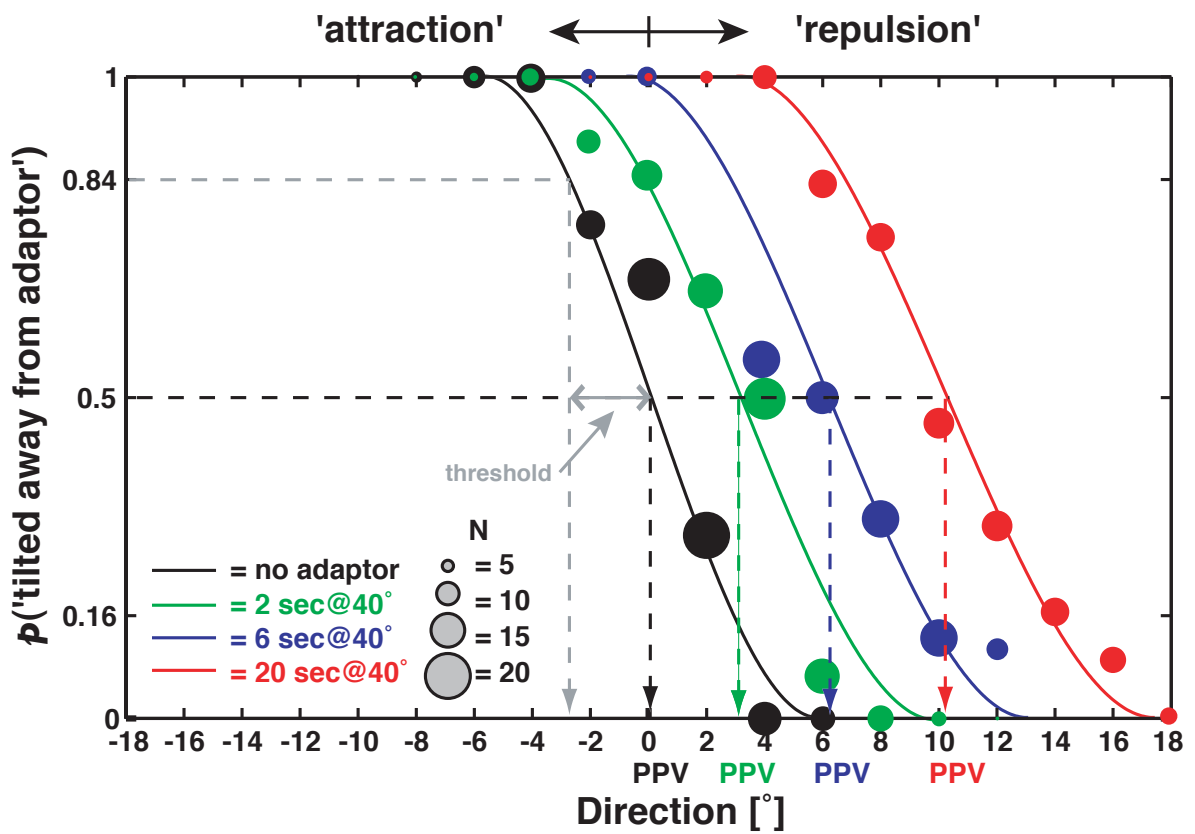

Figure 2: Single subjects ('JEG') judgments about the deviation of the point-of-perceived-vertical (PPV) after $40^{\circ}$ directional motion adaptation for different durations and the baseline ('black') measurement. Positive direction values are used for directions away from vertical towards the adapter and the point of inclination of the fitting curve (50\%-probability; black arrow) is defined as the subjects perceived vertical. The distance between this point and the 84\%-probability point of the fit (1 SD; gray arrow) is taken as discrimination threshold. Different deviations from the vertical direction $\left(0^{\circ} / 180^{\circ}\right)$ had to be judged if they were perceived to move clockwise or counterclockwise. Data from the $0^{\circ}$ and $180^{\circ}$ test conditions is pooled and was collected simultaneously within a session. Colors indicate the respective adaptation condition, whereas the size of the circles represents the number of measurements at the given angular adapter-test difference. 'No adaptation' causes the PPV and the true vertical to be roughly the same as expected. The PPV is shifted to higher values after longer adaptation, which is interpreted as repulsive aftereffect (see text for further explanations).

For '0\%-coherence'- and 'no'-adapter conditions positive values of a indicate a clockwise bias of the subjective vertical. During '100\%-coherent motion'-adaptation positive values of a indicate a shift of the subjective vertical towards the adapted direction, which represents a repulsive DAE.

The respective a and $\sigma_{\text {exp }}$ values of all experiments were statistically analyzed (repeated measures one-way ANOVA; Tukey's multiple comparison test), using "Prism 4.0c" for Macintosh.

\section{Results}

Our experiments focused on the effect of short adaptation durations on the perceived direction of moving random dot patterns deviating by approx. $40^{\circ}$ from the adapted direction. Figure 2 plots the direction discrimination curves for the three adaptation durations and a control condition without adaptation for a single subject. The abscissa represents the direction of the test stimulus in degree of deviation from the vertical. Posi- tive values are used for test directions that are tilted toward the adapter.

The ordinate indicates the percentage of test stimulus presentations for which the subject reported that the test direction's deviation from the vertical pointed away from the adapter's direction. The diameter of each data point represents the number of repetitions for each condition.

The data of each condition are fitted with a logistic function (goodness-of-fit: $p<0.001$ for all datasets).

The $50 \%$ point of each curve represents the test direction for which subjects are equally likely to report the test stimulus to be tilted clockwise or counterclockwise from vertical. This represents the 'point-ofperceived-vertical' (PPV) in a particular condition. Correspondingly, for the subject plotted in Figure 2, the PPV equals the true vertical in the 'no-adapter' condition (black vertical arrow).

The steepness of each curve indicates the sensitivity of the subjects to differences between test directions. We de-fine the angular deviation from the PPV to the point 
3. a)

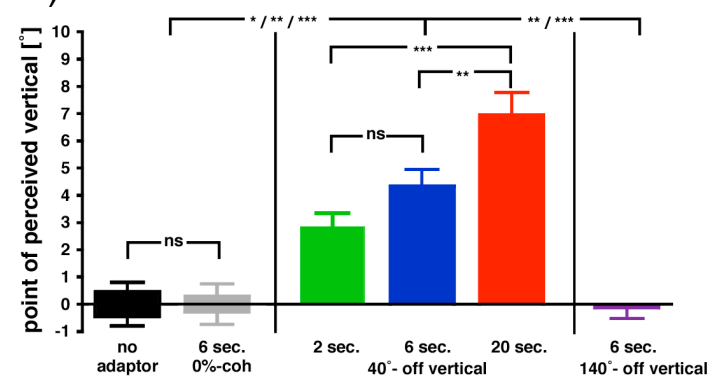

Figure 3: Mean results of all subjects.

a) Shift of perceived vertical: 'No-adaptation' (black) and '6seconds-random-direction-adaptation' (gray) evaluated no bias in the perceived direction of motion. Longer adaptation to $40^{\circ}$ off vertical (green $=2 \mathrm{sec}$; blue $=6 \mathrm{sec}$; red $=20 \mathrm{sec}$ ) caused significant changes in the perceived direction of vertical and thus repulsion. Adapting at $140^{\circ}$ off vertical (magenta) did not significantly alter the perception of the subsequent vertical.

where the responses of subjects exceed 0.84 (or fall below 0.16 ) as the discrimination threshold.

To assess the effect of repulsive aftereffects on direction discrimination curves let us consider the case of a true vertical test direction. Subjects would perceive this stimulus as deviated from the vertical, away from the adapter's direction. Correspondingly, for a stimulus to be perceived as moving exactly vertically it needs in fact be tilted towards the adapter by the amount of repulsion. Such PPVs are plotted as positive values in Figure 2. The observation that the directiondiscrimination curves based on the three adaptation conditions are shifted to the right indicates a repulsive aftereffect for all adapting conditions in this subject. Figure 3 plots the PPV values (Figure $3 a$ ) and thresholds (Figure $3 b$ ) averaged across all subjects. These population averages show the same pattern as the single subject data in Figure 2.

\section{Unadapted Performance}

The bias of the perceived vertical without adaptation for all but one subject was within one standard deviation of the mean (ø:0.45; S.D.:1.09) and did not differ significantly ( $p=0.22,2$-tailed t-test) from zero over all subjects.

Every subject showed a mean un-adapted direction discrimination threshold smaller than $3^{\circ}\left(\varnothing: 2.41^{\circ}\right.$; S.E.: $\left.0.21^{\circ}\right)$.

\section{0\%-coherent motion (random noise) adaptation:}

Adapting with a random direction noise pattern $(0 \%-$ coherent directional motion) for 6 seconds revealed no significant change in the threshold $\left(2.74^{\circ} \pm 0.31^{\circ} \mathrm{SE}\right.$; $p>0.05)$ nor the bias of the center $\left(0.29^{\circ} \pm 0.45^{\circ} ; p>0.05\right)$ b)

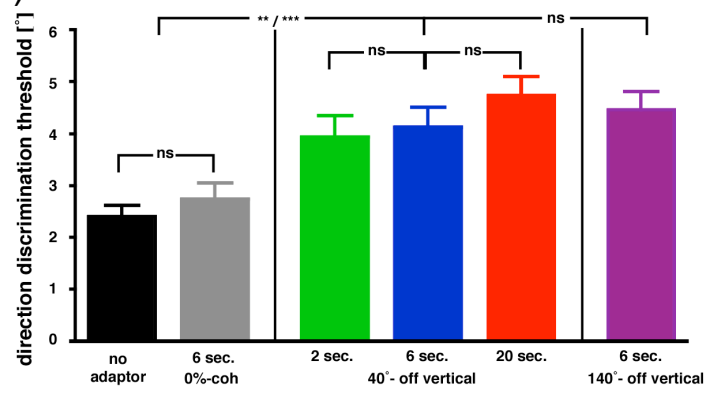

b) Whereas random direction adaptation (gray) did not cause a significant increase in the threshold compared to baseline performance (black), all directional adaptors had a significant influence on the threshold in that they increased it $1.6-2-$ fold. Between the directional adaptor conditions the threshold did not differ significantly.

compared to the un-adapted performance. Also here the distributions of perceived verticals did not differ from zero ( $p=0.57$; 2-tailed t-test).

\section{Directional adaptation: $\mathbf{4 0} \mathbf{~ d e g}$}

After $40^{\circ}$ directional adaptation for 2 seconds all subjects showed a shift of the perceived vertical compared to their baseline performance. Taken together this resulted in a significant repulsive effect $\left(2.84^{\circ} \pm 0.52^{\circ} ; \mathrm{p}<0.05\right)$ in relation to the mean unadapted performance and was also significantly deviating from zero $(p<0.001)$. The mean direction discrimination threshold increased by about $1.5^{\circ}\left(3.94^{\circ} \pm 0.40^{\circ}\right.$; $\mathrm{p}<0.01)$ after 2 seconds of directional adaptation, with an increase in all except one of the individual subjects' data.

After 6 seconds of adaptation every subject's tendency to judge the test-pattern as being repulsed by the adapter increased $\left(4.34^{\circ} \pm 0.61\right)$. This was highly significant over all subjects compared to their baseline $(p<0.001)$ and com-pared to zero $(p<0.0001)$. The increase in average repulsion relative to 2 seconds of adaptation did just miss significance $(p>0.05)$. The discrimination threshold was significantly higher than in the baseline condition $\left(4.12^{\circ} \pm 0.39^{\circ} ; \mathrm{p}<0.001\right)$ but was also not significantly affected by the ex-tended adaptation compared to 2 seconds of directional adaptation duration ( $p>0.05$ ).

As expected, 20 seconds of motion adaptation caused the largest repulsion $\left(6.96^{\circ} \pm 0.82^{\circ} ; \mathrm{p}<0.001\right)$ in every subject. Discrimination thresholds $\left(4.73^{\circ} \pm 0.35^{\circ}\right)$ on the other hand were not significantly higher $(p>0.05)$ compared to the $6 \mathrm{sec}$ adaptation duration. 


\section{Directional adaptation: $140 \mathrm{deg}$}

Adapting 6 seconds $140^{\circ}$ off the vertical had no significant influence - neither repulsive nor attractive - on the PPV $\left(-0.13^{\circ} \pm 0.39^{\circ} ; \mathrm{p}>0.05\right)$ compared to baseline performance and to $0 \%$-coherent motion $(p=0.74)$. Only the threshold was larger compared to the baseline $\left(4.46^{\circ} \pm 0.36^{\circ} ; \mathrm{p}<0.001\right)$ and in a range similar to the $40^{\circ}$ - adaptation.

All directional adapters significantly increased the direction discrimination threshold $(p<0.05$ to $p<0.001)$ but adaptation duration had no significant influence on the magnitude of this effect $(p>0.05)$. Moreover, thresholds in general seem to be affected more strongly by directional motion than by a $0 \%$-coherence pattern. (Figure 3b)

\section{Discussion}

We determined the magnitude of the repulsive direction aftereffect of short-term adaptation in the human visual system. Subjects judged the perceived direction of a moving random dot pattern after brief (2 or 6 seconds) off-axis $\left(40^{\circ}\right)$ adaptation. All subjects showed the classical repulsive effect, i.e., a misperception of the direction of motion away from the adapting direction (Levinson \& Sekuler, 1976). Most importantly, even an adaptation of only 2 seconds created a highly significant aftereffect. This shows that motion adaptation changes directional judgments on a much shorter time-scale than previously reported.

Similarily, we observed highly significant elevations of the direction discrimination thresholds even after as little as 2 seconds of adaptation, and of the same magnitude as reported for long adaptation durations (Hol and Treue, 2001).

Effects of short-term adaptation appear to be a general phenomenon. There are reports of shape aftereffects (Suzuki et al., 2001), speed aftereffects (Krekelberg et al., 2006) and motion aftereffects (rMAE; Kanai and Verstraten, 2005) after 1-2 seconds of adaptation.

No perceptual change after $140^{\circ}$ adaptation: Repulsion effects have been well accounted for by simple fatigue-models of adaptation. The observation of an attractive aftereffect for large angles between adaptor and test-directions (Schrater and Simoncelli, 1998) suggests a need for more elaborate models, since fatigue models can only account for repulsive effects. Such more elaborate models postulate the additional presence of a bandwidth change of neurons that code directions near the adapter (band-width re- duction) and of neurons that prefer direc-tions far from the adapter (band-width increase; Clifford et al., 2000).

Our data, like that of some other studies (Patterson et al.,1996; Levinson \& Sekuler, 1976), do not show an attractive aftereffect. Even Schrater and Simoncelli (1998) point out, that their reported attractive aftereffects may be an artificial interaction between motion- and orientation-mechanisms, because of the use of gratings during adaptation.

\section{Conclusion}

Our data document the existence of rapid adaptation on a timescale fitting the dynamics of everyday vision, i.e., after a couple of seconds or less of exposure.

This is consistent with 'fatigue'-models that interpret adaptation as a neuronal gain-change. It leaves open the possibility, that longer adaptation durations evoke aftereffects that cannot be fully accounted for by such simple gain-change models (Descalzo et al., 2005).

The presence of substantial adaptation effects after brief adaptation significantly enhances the possibilities to study the neurophysiology of adaptation effects in awake fixating monkeys.

\section{Acknowledgements}

We thank Chistine Tillmann for the support during datacollection.

This research was supported by the Volkswagen Stiftung.

Commercial relationships: none.

\section{References}

Barlow, H. \& Földiak, P. (1989). Adaption and decorrelation in the cortex. In: The Computing Neuron, pp. 54-72. Durnin, R. et al. (Eds.) Addison-Wesley Long-man Publishing Co., Inc.

Carandini M. \& Ferster D. (1997). A tonic hyperpolarization underlying contrast adaptation in cat visual cor-tex. Science, 276:949-952.

Clifford C. W. G., Wenderoth P. \& Spehar B. (2000). A functional angle on some aftereffects in cortical vision. Proc. R. Soc. Lond. B, 267:1705-1710.

Clifford C. W. G. \& Rhodes G. (2005). Fitting the mind to the world - Adaptation and aftereffects in high level vision. Oxford University Press.

Curran, W., Clifford, C. W. G. \& Benton, C. P. (2006). The direction aftereffect is driven by adaptation of local 
motion detectors;. Vision Research, 46(25):42704278..

Descalzo V. F., Nowak L. G., Brumberg J. C., McCormick D. A., \& Sanchez-Vives, M. V. (2005). Slow adaptation in fast-spiking neurons of visual cortex. Journal of Neurophysiology, 93(2):1111-1118.

Dragoi V., Sharma J., \& Sur M. (2000). Adaptation-induced plasticity of orientation tuning in adult visual cortex. Neuron, 28:287-298.

Giaschi D., Douglas R., Marlin S. \& Cynader. M. (1993). The time course of direction-selective adaptation in simple and complex cells in cat striate cortex. Journal of Neurophysiology, 70:2024-2034.

Gibson J. J. \& Radner M. (1937). Adaptation, aftereffect and contrast in the perception of tilted lines: I. quantitative studies. Journal of Experimental Psychology, 20:453467.

Hol K. \& Treue S. (2001). Different populations of neurons contribute to the detection and discrimination of visual motion. Vision Research, 41:685-689.

Kaernbach C. (1991). Simple adaptive testing with the weighted up-down method. Perception \& Psychophysics, 49:227-229.

Kanai R. \& Verstraten F. A. J. (2005). Perceptual manifestations of fast neural plasticity: Motion priming, rapid motion aftereffect and perceptual sensitization. Vision Research, 45:3109-3116.

Kohn A. \& Movshon J. A. (2004). Adaptation changes the direction tuning of macaque MT neurons. Nat Neurosci, 7:764-772.

Krekelberg B., van Wezel R. J. A. \& Albright T. D. (2006). Adaptation in macaque MT reduces perceived speed and improves speed discrimination. Journal of Neurophysiology, 95:255-270.

Land, M. (2004). Eye movements in daily life. In Chalupa, L. and Werner, S., editors, The Visual Neurosciences, volume 2, pages 1357-1368. MIT Press

Lennie P. (2003). The cost of cortical computation. Cur-rent Biology, 13:493-497.

Levinson E. \& Sekuler R. (1976). Adaptation alters perceived direction of motion. Vision Research, 16:779-780.

Mather G., Verstraten F. A. J. \& Anstis S., eds. (1998). The Motion Aftereffect: A Modern Perspective. MIT Press.

Movshon J. A. and Lennie P. (1979). Pattern-selective adaptation in visual cortical neurones. Nature, 278:850-852.

Müller J. R. Metha A. B., Krauskopf J. \& Lennie P. (1999). Rapid adaptation in visual cortex to the structure of images. Science, 285:1405-1408.

Patterson R. \& Becker S. (1996). Direction-selective adaptation and simultaneous contrast induced by stereoscopic (cyclopean) motion;. Vision Research, 36(12): 1773-1781.

Petersen S. E., Baker J. F. \& Allman, J. M. (1985). Directionspecific adaptation in area MT of the owl monkey. Brain Research, 346:146-150.

Regan D. and Beverly K. (1985). Postadaptation orientation discrimination. J Opt Soc of America A, 2:147-155.

Schrater P. R. \& Simoncelli E. P. (1998). Local velocity representation: evidence from motion adaptation. Vision Research, 38:3899-3912.

Suzuki S. (2001). Attention-dependent brief adaptation to contour orientation: a high- level aftereffect for convexity ? Vision Research, 41:3883-3902.
Thompson P. (1981). Velocity aftereffects: The effects of adaptation to moving stimuli on the perception of subsequently seen moving stimuli. Vision Research, 21(3):337-345.

Wohlgemuth (1911). On the aftereffect of seen movement. British Journal of Psychology, 1:1-117 
40CHAPTER 2. BRIEF MOTION ADAPTATION CAUSES REPULSIVE AFTEREFFECTS -PREPARE 


\section{Chapter 3}

\section{Response saturation determines attentional modulation onset in MT}

\section{neurons}

In his groundbreaking book "Vergleichende Lokalisationslehre der Grosshirnrinde in ihren Prinzipien dargestellt auf Grund des Zellenbaues" the german anatomist Brodmann (1909) used the variation of number and - anatomical - type of neurons in the cortex to differentiate areas in the brain. Nowadays, we know that these structural differences also represent functional differences, i.e. areas represent specific sensory-, association- or motor-features. Additionally, the cortical neurons differ in their specific interconnectivity (Roerig et al., 2003; Stettler et al., 2002; Douglas and Martin, 2004) and cell-types can be classified by either morphological or/and physiological parameters in almost unmanageable ways (Kawaguchi, 1995; Shinomoto et al., 2003; Nowak et al., 2003; Markram et al., 2004).

Despite this diversity, the neurons recorded in one cortical area are usually mostly implicitly - assumed to build a homogeneous, ergodic population (Masuda and Aihara, 2003). However, whenever one lowers an electrode into the brain one does not exactly know, which type of neuron one records from, although, there is a bias toward principal cells due to their larger and further-spreading extracellular potentials (Towe and Harding, 1970). Additionally, there is a bias towards cells with relatively high spontaneous firing rate and most cells even do not leave their electrical 'fingerprint' on the electrode tip (Shoham et al., 2006). In summary, one has to assume, that the population of neurons that is represented in a given data-set is composed of different neuron-types (Mitchell et al., 2007) with different response 
properties.

In such a population of MT neurons, recorded under various attentional conditions, we found a general temporal delay in the attentional modulation after stimulus onset. We determined, if and how far the time-course of the attentional top-down modulation of each cell varies with its bottom-up sensory response characteristics and found that the attentional delay was indeed not equivalent across all cells in the population. Moreover, the response saturation of some cells during their boosting initial bottom-up response seemed to prevent a further attentional up-regulation of the spike-rate during the early phase of the response. Cells with a more tonic response profile were immediately affected by the top-down modulation. 


\title{
Response Saturation determines Attentional Modulation onset in MT Neurons (prepared for submission)
}

\author{
Florian Pieper ${ }^{1}$, Thilo Womelsdorf ${ }^{2}$, Dieter Patzwahl $^{3}$, Stefan Treue ${ }^{1} \&$ Julio C. Martinez-Trujillo ${ }^{4}$ \\ ${ }^{1}$ German Primate Center, Kellnerweg 4, 37077 Goettingen, Germany \\ 2 F.C. Donders Centre for Cognitive Neuroimaging, Radboud University Nijmegen, Kapittelweg 29, 6525 EN Nijmegen, The Netherlands \\ ${ }^{3}$ Multichannel Systems, Apenhaustrasse 21, 72770 Reutlingen, Germany \\ ${ }^{4}$ Department of Physiology, McGill University, McIntyre Medical Sciences Building, 3655 Promenade Sir William Osler, Montreal, \\ H3G 1 Y6 QC, Canada
}

\begin{abstract}
Visual attention modulates information processing in the visual cortex of primates by increasing neuronal responses to attended stimuli relative to unattended ones. We have found that for neurons in middle temporal area (MT) of macaques such response increase arises on average at about $120 \mathrm{~ms}$ after a stimulus appeared at an attended location ('attentional delay'). However, at the level of individual neurons attentional delay varies considerably. Here, we demonstrate that such variability is largely determined by the relationship between the transient-to-sustained response magnitudes (transient index). Neurons with larger transient indices show practically no attentional modulation during the first $150 \mathrm{~ms}$ after a visual stimulus onset resulting in large attentional delays. On the other hand, neurons with low transient indices were significantly modulated by attention immediately after response onset.
\end{abstract}

Keywords: transient response, transiency, contrast gain, instantaneous spike rate, feature-based attention, transparent motion

Corresponding author:

Florian Pieper

German Primate Center

Cognitive Neurosciences Laboratory

Kellnerweg 4

37077 Goettingen

GERMANY

email: fpieper@gwdg.de

phone:++49(0)551-3851-345

fax: $\quad++49(0) 551-3851-452$

\section{Introduction}

Visual attention increases the detectability and discriminability of stimuli in the visual field. Single-unit recordings from behaving macaque monkeys have show that the neural correlate of these effects is an enhanced response of single neurons in primate visual cortex to attended compared to unattended stimuli (Moran \& Desimone, 1985; Spitzer et al., 1988; Haenny et al. 1988a,b; Motter, 1993, Connor et al., 1996; Luck et al., 1997; Treue \& Maunsell, 1996,1999; Treue \& Martinez-Trujillo, 1999; McAdams \&Maunsell, 1999; Fries, 2001; Reynolds et al., 1999). On the level of neuronal populations, the temporal dynamics of this effect with respect to the appearance of the stimuli has been previously described: immediately after stimulus onset, the average neuron is apparently not modulated by attention, but as soon as the initially boosting response adapts to a lower, sustained response, the strength of the attentional effect increases to its maximum. Different authors have reported different starting times for the attentional response modulation (attentional delay): For V4-neurons such attentional delays are in the range of 150-200ms (Motter, 1994a; Reynolds et al., 2000; Chelazzi et al., 2001). For V1, Roelfsema et al. (1998) found an attentional delay of about $235 \mathrm{~ms}$ and Seidemann \& Newsome (1999) one of $\sim 250 \mathrm{~ms}$ for MT neurons.

At least two factors may underlay this variability of the attentional delay. The first one is a behavioral delay, i.e. attention might only be allocated after the stimulus appearance in a given task design (top-down bias). The neural correlate of such a close temporal link between the instantaneous level of attentional load (determined by the hazard rate, i.e. the momentary likelihood of a behaviorally relevant event) and the 
modulation of neuronal responses has been demonstrated recently (Ghose \& Maunsell (2002), Janssen \& Shadlen (2005)).

The second possibility is a difference in the susceptibility of neurons to the modulatory effect of attention during different response periods (bottom-up or sensory bias). A transient response saturation after the onset of a stimulus could be this alternative reason for the attentional delay. It has been proposed that response saturation evoked by high contrast stimuli makes individual neurons less sensitive to attentional modulation (Martinez-Trujillo \& Treue, 2002; Reynolds et al., 2000; Treue, 2001). Therefore, neurons with a strong transient response (figure 1b) and therefore more likely near their maximally possible activation are hypothesized to show a larger attentional delay since the attentional influence becomes apparent only after the transient response, while tonic cells (figure 1c) would be able to exhibit the attentional influence earlier after stimulus onset.

To distinguish between the two alternatives we recorded single cell responses in area MT during an attentional task and examined the temporal dynamic of attentional modulation in neurons with different transient response intensities. We found that attentional delays were indeed strongly correlated with the intensity of transient responses i.e., they were larger in high transient cells relative low transient cells. The different temporal characteristics of individual neurons' response saturation therefore determines the population's average attentional delay.

\section{Methods}

Subjects: We recorded the responses of direction-selective cells to moving random dot patterns (RDP) in area MT of two male monkeys (Macaca mulatta) while the animals performed a speed change detection task. Two different experiments were independently conducted (see below) in both animals. All the experimental procedures were in agreement with the local and national rules and regulations, approved by the Regierungspraesidium Tuebingen, Germany.

Apparatus and recording procedures: The experimental procedures have been described in more detail elsewhere (Martinez-Trujillo \& Treue, 2004). Briefly, the monkeys sat in front of a computer monitor with their head restrained. Eye-positions signals were measured throughout the trials using the scleral-search coil technique (Judge, 1980; Robinson, 1963). A cus- tom computer program controlled the stimulus presentation, monitored and stored the eye position and behavioral responses as well as the neuronal (spiketimes) data during the experiments. The latter were recorded using tungsten microelectrodes (impedance 0.5-2 m $\Omega$, Microprobe Inc. and FHC Inc., USA) advanced into area MT by a hydraulic microdrive (Kopf Instruments, USA) through a craneotomy on the parietal bone. Signals were amplified and classified as spikes using a window-discriminator (BAK Inc, USA).

Stimuli and task: We used random dot patterns (RDP) composed of white dots on a dark background (maximum contrast). The RDPs had a circular shape and a dots density of 5 dots/degree ${ }^{2}$. Their diameter and speed were optimized to match the size and preferred speed of a neuron's receptive field (RF). During the experimental trials, the animals were required to maintain fixation within $1^{\circ}$ of a fixation spot. If they failed to do so the trial was immediately aborted.

In general, an experimental trial consisted of the following sequence of events: A central fixation spot and a static RDP appeared on the computer screen, The monkey foveated the fixation spot and pressed a lever to trigger the trial presentation. 200-400ms after level press the static RDP (target) began to coherently move and a second moving RDP (distractor) appeared on the screen. The animal was rewarded with a drop of juice for releasing the lever in response to a transient speed change in the target while ignoring changes in the distractor. Changes in the target or distactor could appear within a temporal window of $260 \mathrm{~ms}$ to 2300 ms after stimulus onset and they never occurred simultaneously.

We presented two RDPs of equal size at equal eccentricity on opposite sides (hemifields) of the fixation point. One pattern was positioned inside the recorded cell's RF and the other outside. We recorded the responses to 12 different directions of the pattern inside the RF when the target was the RDP positioned outside and when it moved either in the same (attend same) or in the opposite (attend opposite) direction (figure 1a).

In experiment 2 (Supplementary figure 2a) the trial sequence was similar to the one of experiment 1 . However, here the two RDPs were superimposed and moved in opposite directions (transparent motion) inside a neuron's RF. One pattern always moved in the preferred and the other in the opposite/null direction. A cue RDP presented at the beginning of the trial indicated the target pattern ('surface') and disappeared 

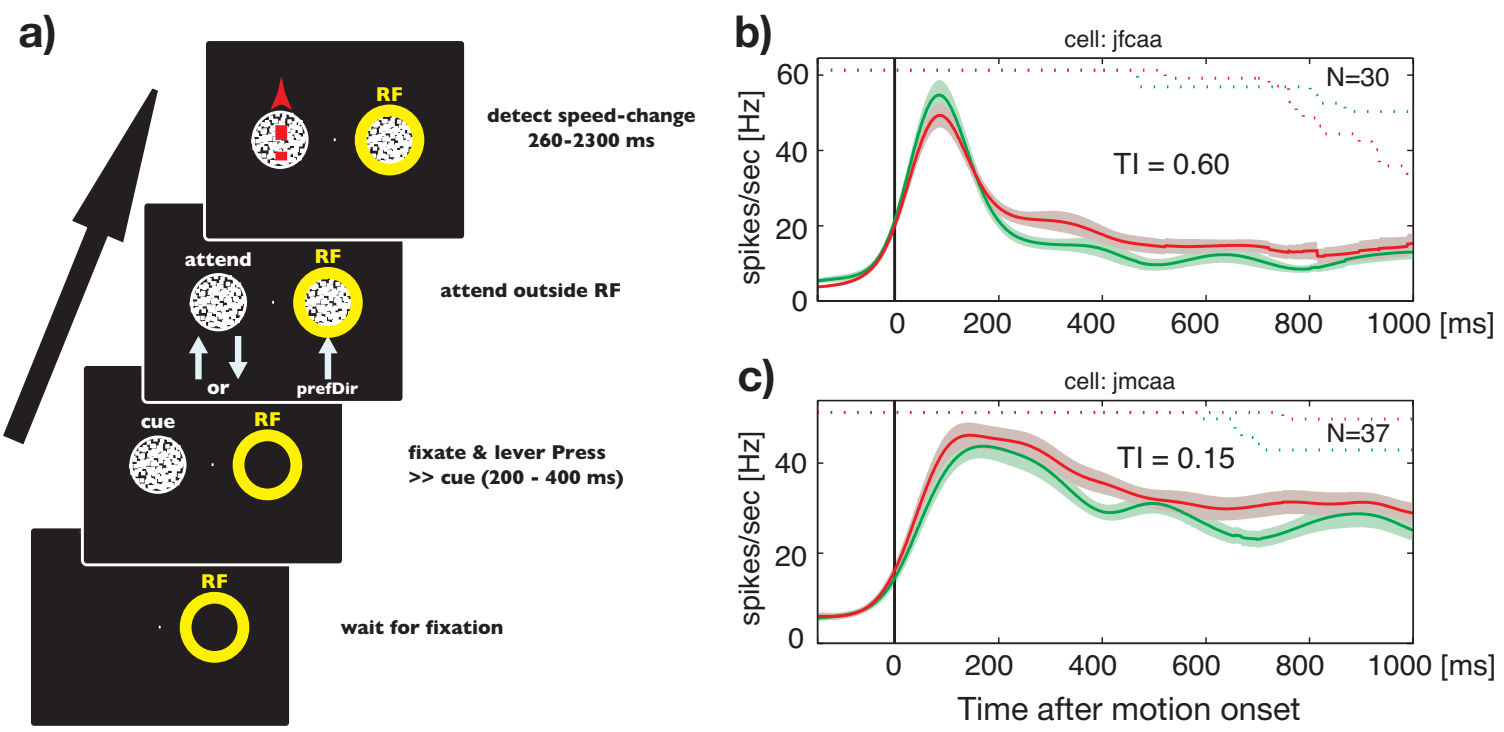

Figure 1: a) Sequence of events in a single trial in experiment 1. After the monkey initiated fixation on the fixpoint, a static spatial cue appeared to cue spatial attention. Then the monkey pressed a lever and the another stimulus appeared on the monitor inside the RF of the neuron we recorded from. Both stimuli began to move and the monkey had to detect a speed change at the cued position ('target') while ignoring changes of the RDP inside the RF ('distractor'). Throughout the whole experimental session, both, inside-RF- and outside-RF-stimuli could be target from trial to trail. Also the directions of motion of the RDP inside the RF was balanced during the session. We restricted our analysis on trials with attention outside the RF and a RDP inside the RF moving in the neurons preferred direction as highlighted by the arrows. This is the same condition as shown in figure $2 a$ of (Treue and Martinez-Trujillo,1999) and figure 1a (left) of (Martinez-Trujillo \& Treue,2004). b) Response of a highly transient cell to a preferred direction stimulus inside ist RF, when the spatially attended stimulus outside the RF moved either in the same (red) or opposite (green) direction. c) Example of a low transient cell from the same experiment 1.

$350 \mathrm{~ms}$ before the transparently moving RDPs appeared on the screen. The monkey was instructed to detect a speed change in the target surface and ignore similar changes of the distractor surface. We compared responses when the monkey attended to the pattern moving in the preferred direction against responses when the animal ignored the RDPs and had to detect a luminance-change at the fixation spot.

Data analysis: For the data of experiment 1 we determined the direction tuning of every cell by fitting average responses to a circular wrapped Gaussian distribution (Bratschelet, 1981):

$$
R(\theta)=B+\sum_{i=-2}^{2} A^{\left(\frac{-(\theta-\phi-i * 360)^{2}}{\sigma^{2}}\right)}
$$

where $R$ is the fitted response to a stimulus direction $\Theta, B$ is the offset (minimum) of the response, $A$ is the amplitude of the tuning curve and the parameters $\Phi$ and $\sigma$ are the preferred direction and tuning width respectively.

We recorded cell responses and analyzed them over different time periods. We computed a directional index (DI) during the 1200 ms after stimulus onset using the equation:

$$
D I=\frac{\text { Response }_{\text {pref. }}-\text { Response }_{\text {anti-pref. }}}{\text { Response }_{\text {pref. }}+\text { Response }_{\text {anti-pref. }}}
$$

In order to increase the number of trials per condition, we pooled trials from directions that were $\pm 30^{\circ}$ away from the preferred and null direction, respectively.

We calculated a neuron's instantaneous spike rate from $350 \mathrm{~ms}$ before to $1200 \mathrm{~ms}$ after stimulus onset in each trial for each interspike-interval (ISI) using the equation:

$$
\operatorname{spikerate}_{(i)}=\frac{1000}{I S I_{(i)}}
$$

For trials with no spikes the spikerate is set to zero rate. To account for between trial spike-timing differences, we convoluted this state-histogram with a 50 ms Gaussian kernel.

Attentional modulation. We computed the percentage attentional modulation (AM) by using the formula:

$$
A M[\%]=\frac{\text { Response }_{\text {attend-same }}}{\text { Response }_{\text {attend }- \text { opposite }}} * 100
$$

Two time windows were used for this computation: a) from 50 to $250 \mathrm{~ms}$ after motion-onset (transient response, 'early'), and b) from 300 to $1000 \mathrm{~ms}$ after motion onset (sustained response, 'late'). We analyzed 


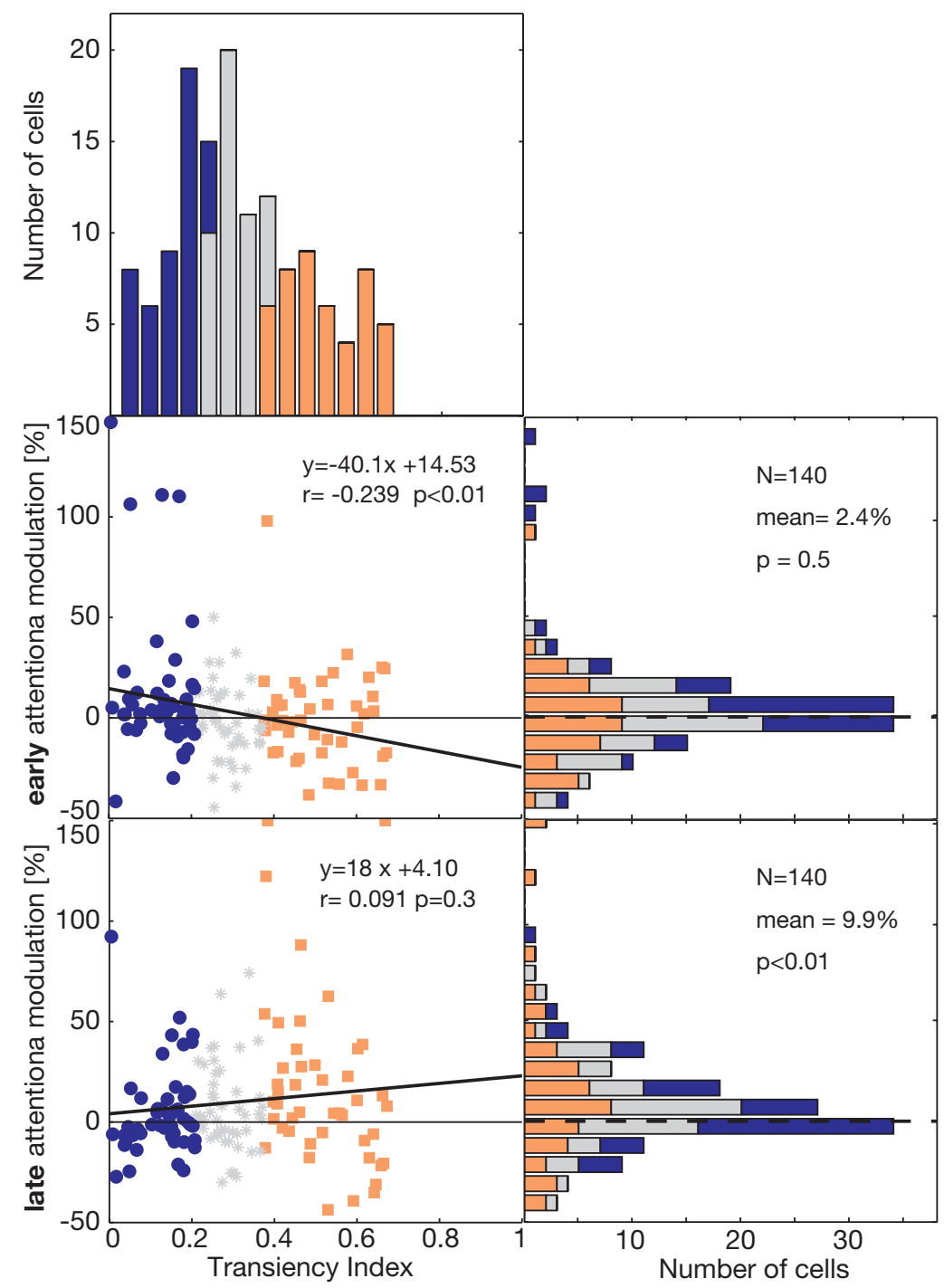

Figure2: Statistics of the early (middle plots) and late (lower plots) attentional modulation of 140 neurons with respect to their transiency (upper plot). The colors indicate the membership to either the low-, medium- and high-transient subpopulation (blue, gray, orange) as referenced in the rest of this publication. The Tls are normal distributed. During the early response period the cells are at mean not modualted (ø: $2.4 \% ; p=0.5)$ by attention. However, the regression $(p<0.01)$ through the AM as function of the TI show, that low transient cells are likely to be positively attentional modulated while high transient cells show a slightly negative AM during this period with attention. This relationship is no more present $(p=0.3)$ during the late, sustained response period. Here, in contrast to the early period, the mean AM is significantly positive at $9.9 \%(p<0.01)$.

only cells that exhibited a mean positive AM during the late period.

Transient-to-sustained index (TI). This was computed by using the formula:

$$
T I=\frac{\text { Response }_{\text {init. }}-\text { Response }_{\text {sust }} .}{\text { Response }_{\text {init. }}+\text { Response }_{\text {sust }}}
$$

Cells were sorted according to their $\mathrm{Tl}$ and pooled into two classes representing either $33 \%$ low- or high Tls. Figure 1 shows examples of neurons with high (c) and low (d) transient indices, respectively.

Attentional latency was defined as the time from motion onset after which the median AM was always positive. We divided neurons into five groups according to their $\mathrm{Tl}$ and then computed a bootstrap median over the mean attentional time-courses of each group. From this we determined the attentional latencies and fitted a regression line through these values.

\section{Results}

We recorded the responses of 170 MT neurons to the same distractor stimulus inside their RF while the animals switched attention between two different motion directions of a target stimulus outside. We restricted our analysis to the 139 neurons with Dls larger than 
a)

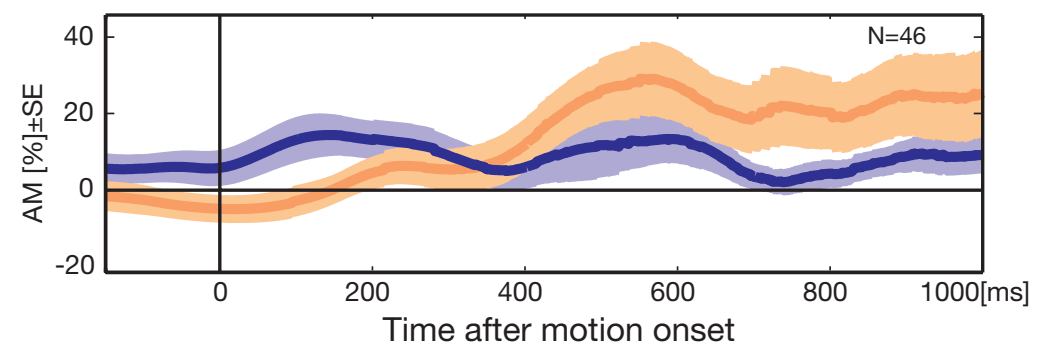

b)

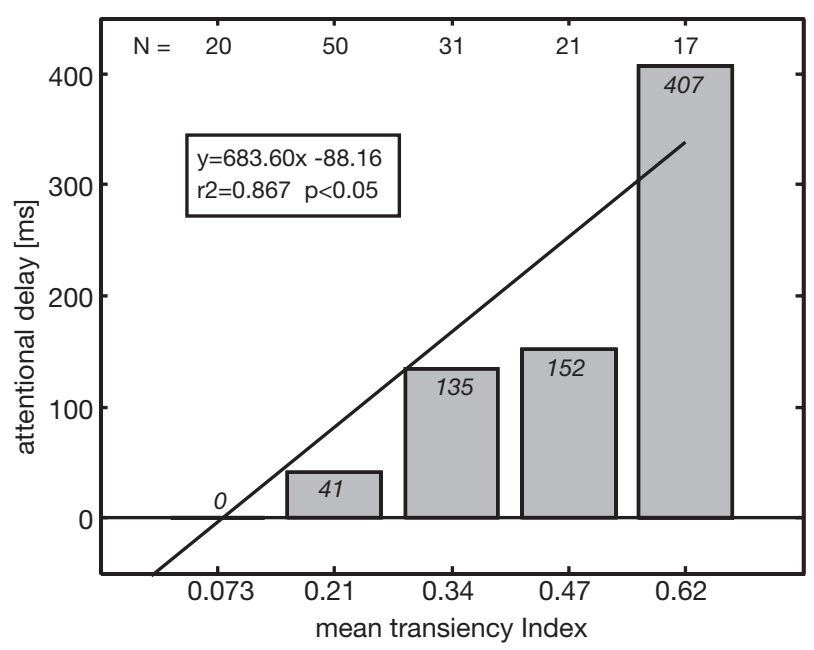

Figure 3: a) Average time course of the attentional effect in the low (b/ue) and high (orange) transient sub-pupulation. While the low transient cells are positively modulated by attention immediately with the appearance of the moving stimuli on the screen, the high transient cells' AM is delayed by about $180 \mathrm{~ms}$ until it constantly stays positive. (see Supplementary Fig $2 \mathrm{~d}$ for similar effects in experiment 2)

b) Linear regression of the attentional latency as a function of transiency index for experiment 1. All 5 bins span an equal range of the whole populations TI range. The number of cells evaluated in each bin is depicted over the bins. The respective latency value in $\mathrm{ms}$ is assigned to every bin.

0.3 , with at least 8 correctly performed trials per atten-

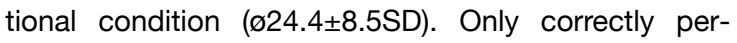
formed trials were analyzed. In this group Tls varied from 0 to 0.67 and showed a unimodal distribution (D'Agostino-Pearson omnibus test; $p=0.10$ ) with a mean of $0.31 \pm 0.17 \mathrm{SD}$ (figure 2a).

In order to quantify the effects of feature-based attention across our sample we computed the attentional modulation (AM) within two time windows ('early' and 'late', see methods). The mean late AM was $10 \%$ $(9.9 \pm 3 \% \mathrm{SE} ; \mathrm{p}<0.01$, Wilcoxon signed rank), i.e. the neurons' responses were enhanced when the animal was attending to the preferred direction of motion (figure $\mathbf{2 a}$, histograms on the right) but the mean early AM was not different from zero $(2.5 \pm 2.4 \% \mathrm{SE} ; \mathrm{p}=0.5$, Wilcoxon signed rank). We fitted a linear regression model to the early and sustained AM data as a function of the $\mathrm{TI}$ in all neurons. During the early period, cells with higher Tls were clearly less modulated than those with lower Tls (figure 2a-middle-left: slope $=-40 ; 95 \% \mathrm{Cl}=$ -66 to -13$)$. However, during the late period the same neurons show no correlation between $\mathrm{TI}$ and $\mathrm{AM}$ (figure 2a bottom-left: slope $=18 ; 95 \% \mathrm{Cl}=-16$ to 53 ). This suggests that during the transient period cells with the largest TI were less modulated by attention. The individual cells relationship between early and late $\mathrm{AM}$ can be seen in the supplementary figure $\mathbf{1 a}$.

For a closer evaluation of this observation we examined the time course of the attentional modulation in cells with high and low Tls. We divided our sample into three equally sized groups (low $(0<\mathrm{Tl}<0.21)$, mid $(0.21<\mathrm{Tl}<0.37)$, and high $(0.38<\mathrm{Tl}<0.67)$ transient cells) with 48 neurons each. Each sub-group's mean modulation during the two periods is shown in the supplementary figure $\mathbf{1 b}$. The average AM over time for low and high transient cells is plotted in figure $\mathbf{3 a}$. Clearly, 
AM becomes positive in the low transient group much earlier than in the high transient group. Interestingly the AM in low transient cells remains almost constant over time during.

In order to further test whether TI determines attentional delay we grouped cells according to their $\mathrm{TI}$ into 5 different sub-groups of equal TI-range to reduce variability in our estimates of attentional delays for individual cells and determined the attentional latency in each sub-group (figure $\mathbf{3 b}$ ). Across the sub-groups attentional delays become shorter for cells with low Tls and the regression line relating the attentional delay with $\mathrm{TI}$ shows a significant positive slope (abscissa = -88 , slope $=683, p<0.05$, F-test). This suggests that indeed the transient index determined the attentional delay in our sample of MT neurons.

One possibility that could account for this result is that neurons with low and high $\mathrm{Tl}$ showed different selectivities for motion direction and since we are examining the effect of attention to the cell preferred direction (feature-based attention) this variable could be correlated with the intensity and onset of attentional modulation (Martinez-Trujillo \& Treue, 2004). Thus, we examined the directionality of low and high TI cells. These two groups had similar selectivity for motion direction (mean $\mathrm{DI}=0.84$ for low and 0.85 for high transient, $\mathrm{p}=0.81$, t-test, data not shown). So, we conclude that directional-selectivity cannot account for the observed results.

The results of experiment 2 point in the same direction like those reported for experiment 1 . Please, refer to the supplementary material for the detailed results.

Taken together, both experiments showed a significant correlation between the transiency of the response and the initial susceptibility to attentional modulation, such that higher transiency was accompanied with a lower initial $\mathrm{AM}$ and a longer attentional delay (experiment 1 only).

\section{Discussion}

The most important finding in this study is that the relationship between transient and sustained responses in MT neurons determines the onset of attentional modulation (attentional delay) in the presence of a constant hazard rate. The bigger the difference between the initial (transient) response within a 50-250ms time-window after stimulus onset and the sustained response thereafter, the higher the attentional delay. In contrast neurons with low transient to sustained response differences showed the shortest attentional delays.

\section{The hazard rate}

Our results are very unlikely explained by systematic variations of the attentional load over the period of the trial due to a monkey's changing expectancy of a critical event in the target stimulus, i.e. the hazard rate (Ghose \& Maunsell, 2002; Janssen \& Shadlen, 2005). This is because in our experiments the hazard rate was always kept constant. Interestingly, despite the hazard rate was zero during the first $260 \mathrm{~ms}$ of stimulus presentation in both experiments, many cells were AM much earlier. The most likely explanation for this effect is that the animals voluntarily chose to allocate attention to the stimulus early during the trial since the cue presented at the beginning of the trial indicated the attended direction of motion from trial onset. Thus, it was likely not a good strategy for the animal to direct attention away from the stimulus direction and then re-allocate it $260 \mathrm{~ms}$ later on the same stimulus. We therefore conclude that the reported effect is caused by differences in the transient to sustained response across individual neurons.

\section{Response saturation}

Another important implication of our result is for current models of attentional modulation (contrast vs. response gain). It has been proposed that attention is a gain regulating mechanism that multiplicatively changes neuronal responses across the cortex (McAdams \& Maunsell, 1999; Treue \& MartinezTrujillo,1999). Some authors have further proposed that the effects of attention are similar to the effect of stimulus contrast (Reynolds et al. 2000; MartinezTrujillo \& Treue,2002) and therefore hypothesized that attention modulates sensory inputs into visual neurons before contrast saturation has occurred. However, other authors have failed to find a systematic saturation of attentional modulation and therefore proposed that attention is simply a mechanism that multiplies the cell response (Williford \& Maunsell, 2006). Our results are in agreement with the former hypothesis since clearly for high transient neurons attentional modulation was virtually absent during the transient response period (see figure 2).

\section{Mechanisms}

One possible explanation for our findings would be that in high-transient cells the synaptic transmission is already saturated by the sensory feed-forward input of a high-rate neuron into the recorded MT cell, which 
in turn causes synaptic depression in the latter. This effect of non-linear summation of EPSP's has been described for V1 neurons receiving LGN input (Tsodyks \& Markram, 1997; Abbott et al., 1997; Varela et al., 1997) and also modeled by Chance et al. (1998). If the attentional modulation directly affects synaptic inputs, when the synapse is saturated (during the transient response in high transient cells) and therefore falls into synaptic depression, attention cannot further increase synaptic transmission. In the low transient neurons the spike rate of the inputs is within a range that does not cause synaptic depression. Hence, they are immediately modulated by attention. In fact Olshausen et al. (1993) already proposed such a pre-synaptic gain control and gating mechanism that dynamically 'guides' the information-flow under attention. However, this hypothesis needs further testing.

Interestingly, the attentional modulation of visual neurons also seems to be affected by other individual cell properties. For example Mitchell et al. (2007) recently reported differences in the AM of V4 neurons with respect to the shape of the individual neurons spike-wave-forms. How their result is related to the one reported here is a matter of further research.

\section{Conclusion}

Finally, we conclude that in our sample of MT neurons attentional modulation onset was clearly dependent on the relationship between transient and sustained response in individual cells. This result makes an important insight into the mechanisms of attention in the primate brain.

\section{References}

Abbott, L. F., Varela, J. A., Sen, K., and Nelson, S. B. (1997). Synaptic depression and cortical gain control. Science, 275:221-224.

Bratschelet, E. (1981). Circular statistics in biology. Academic Press.

Chance, F. S., Nelson, S. B., and Abbott, L. F. (1998). Synaptic depression and the temporal response characteristics of v1 cells. Journal of Neuroscience, 18(12):47854799.

Chelazzi, L., Miller, E. K., Duncan, J., and Desimone, R. (2001). Responses of neurons in macaque area v4 during memory-guided visual search. Cerebral Cortex, 11(8):761-772.

Connor, C. E., Gallant, J. L., Preddie, D. C., and Van Essen, D. C. (1996). Responses in area v4 depend on the spatial relationship between stimulus and attention. Journal of Neurophysiology, 75:1306-1308.

Fries, P., Reynolds, J. H., Rorie, A. E., and Desimone, R. (2001). Modulation of oscillatory neuronal synchronization by selective visual attention. Science, 291(5508):1560-1563.
Ghose, G. M. and Maunsell, J. H. R. (2002). Attentional modulation in visual cortex depends on task timing. Nature, 419:616-620.

Haenny, P. E., Maunsell, J. H. R., and Schiller, P. H. (1988). State dependent activity in monkey visual cortex. Experimental Brain Research, 69:245-259.

Haenny, P. E. and Schiller, P. H. (1988). State dependent activity in monkey visual cortex i. Experimental Brain Research, 69:225-244.

Janssen, P. and Shadlen, M. N. (2005). A representation of the hazard rate of elapsed time in macaque area lip. Nat Neurosci, 8:234-241.

Judge, S. J., Richmond, B. J., and Chu, F. C. (1980). Implantation of magnetic search coils for measurement of eye position: An improved method. Vision Research, 20(6):535-538.

Luck, S. J., Chelazzi, L., Hillyard, S. A., and Desimone, R. (1997). Neural mechanisms of spatial selective attention in areas v1, v2, and v4 of macaque visual cortex. Journal of Neurophysiology, 77:24-42.

Martínez-Trujillo, J. C. and Treue, S. (2002). Attentional modulation strength in cortical area $\mathrm{mt}$ depends on stimulus contrast. Neuron, 35:365-370.

Martínez-Trujillo, J. C. and Treue, S. (2004). Feature-based attention increases the selectivity of population responses in primate visual cortex. CurrentBiology, 14:744-751.

McAdams, C. J. and Maunsell, J. H. R. (1999). Effects of attention on orientation-tuning functions of single neurons in macaque cortical area v4. Journal of Neuroscience, 19:431-441.

Mitchell, J. F., Sundberg, K. A., and Reynolds, J. H. (2007). Differential attention-dependent response modulation acrosss cell classes in macaque vusual area v4. Neuron, 55:131-141.

Moran, J. and Desimone, R. (1985). Selective attention gates visual processing in the extrastriate cortex. Science, 229(4715):782-784.

Motter, B. (1994). Neural correlates of attentive selection for color or luminance in extrastriate area v4. Journal of Neuroscience, 14:2178-2189.

Motter, B. C. (1993). Focal attention produces spatially selective processing in visual cortical areas v1, v2, and v4 in the presence of competing stimuli. Journal of $\mathrm{Neu}$ rophysiology, 70:909-919.

Olshausen, B., Anderson, C., and Van Essen, D. (1993). A neurobiological model of visual attention and invariant pattern recognition based on dynamic routing of information. Journal of Neuroscience, 13(11):47004719.

Reynolds, J. H., Chelazzi, L., and Desimone, R. (1999). Competitive mechanisms subserve attention in macaque areas v2 and v4. Journal of Neuroscience, 19:17361753.

Reynolds, J. H., Pasternak, T., and Desimone, R. (2000). Attention increases sensitivity of $v 4$ neurons. Neuron, 26:703-714.

Robinson, E. (1963). A method of measuring eye movement using a scleral search coil in a magnetic field. IEEE Trans. Biomed. Eng., 10:137 - 145.

Roelfsema, P. R., Lamme, V. A. F., and Spekreijse, H. (1998). Object-based attention in the primary visual cortex of the macaque monkey. Nature, 395:376-381.

Seidemann, E. and Newsome, W. T. (1999). Effect of spatial attention on the responses of area mt neurons. Journal of Neurophysiology, 81(4):1783-1794. 
PIEPER et al.: Response Saturation determines Attentional Modulation onset in MT Neurons (prep. for subm.)

Spitzer, H., Desimone, R., and Moran, J. (1988). Increased attention enhances both behavioral and neuronal performance. Science, 240:338-340.

Treue, S. and Martínez-Trujillo, J. C. (1999). Feature-based attention influences motion processing gain in macaque visual cortex. Nature, 399:575-579.

Treue, S. and Maunsell, J. H. R. (1996). Attentional modulation of visual motion processing in cortical areas $\mathrm{mt}$ and mst. Nature, 382(6591):539-541.

Treue, S. and Maunsell, J. H. R. (1999). Effects of attention on the processing of motion in macaque middle temporal and medial superior temporal visual cortical areas. Journal of Neuroscience, 19:7591-7602.

Tsodyks, M. V. and Markram, H. (1997). The neural code between neocortical pyramidal neurons depends on neurotransmitter release probability. Proceedings of the National Academy of Sciences, 94(2):719-723.

Varela, J. A., Sen, K., Gibson, J., Fost, J., Abbott, L. F., and Nelson, S. B. (1997). A quantitative description of short-term plasticity at excitatory synapses in layer 2/ 3 of rat primary visual cortex. Journal of Neuroscience, 17(20):7926-7940.

Williford, T. and Maunsell, J. H. R. (2006). Effects of spatial attention on contrast response functions in macaque area v4. Journal of Neurophysiology, 96(1):40-54. 


\title{
Supplementary Material:
}

\section{Response Saturation determines Attentional Modulation onset in MT Neurons} (prepared for submission)

\author{
Florian Pieper ${ }^{1}$, Thilo Womelsdorf ${ }^{2}$, Dieter Patzwahl $^{3}$, Stefan Treue ${ }^{1} \&$ Julio C. Martinez-Trujillo ${ }^{4}$ \\ ${ }^{1}$ German Primate Center, Kellnerweg 4, 37077 Goettingen, Germany \\ ${ }^{2}$ F.C. Donders Centre for Cognitive Neuroimaging, Radboud University Nijmegen, Kapittelweg 29, 6525 EN Nijmegen, The Netherlands \\ 3 Multichannel Systems, Apenhaustrasse 21, 72770 Reutlingen, Germany \\ 4 Department of Physiology, McGill University, McIntyre Medical Sciences Building, 3655 Promenade Sir William Osler, Montreal, \\ H3G 1 Y6 QC, Canada
}
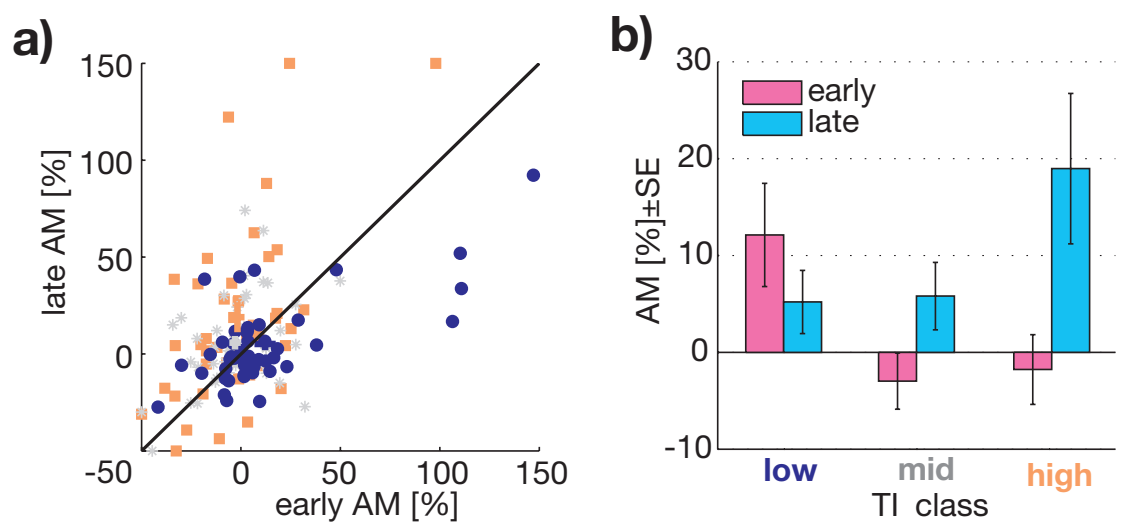

Supplementary Figure 1: Experiment 1

a) Relationship of the individual cells 'early' and 'late' attentional modulation in experiment 1 . The data-points are colored according to their membership to the respective $\mathrm{TI}$ classes (blue = low TI trimester; gray = middle TI trimester; orange = high $\mathrm{TI}$ trimester; $N=140)$.

b) Mean \pm SE attentional modulation of the low-, mid- and high-transient sub population, either in the 'early' (magenta) or 'late' (cyan) response period.

\section{Results Experiment 2:}

During this experiment we recorded 75 neurons. 12 cells were discarded from further analysis, because the number of trials per either condition was lower than six or another criterion already mentioned for experiment 1 was not reached. The two attentional conditions had systematically different numbers of trials $(\varnothing 44.4 \pm 15.8$ SD and $\varnothing 11.6 \pm 4$ SD, respectively). Tuning data was no more available. However, the 'preferred' stimuli evoked substantially larger responses than the 'null'-stimuli. The distribution of Tls of the remaining 63 cells was not normal but left-skewed to lower values.

Here, both, the mean early and late AM were significantly positive across the cells $(17.2 \pm 3.8 \% \mathrm{SE}$; $p<0.001$. and $21.6 \pm 3.2 \% S E ; p<0.001$ Wilcoxon signed rank, Supplementary figure 3 right). However, the linear regression model of the individual cells early and late attentional modulation as function of the TI revealed opposite signed slopes: while the early AM significantly decreased with increasing TI (Supplementary figure 3-middle-left: slope $=-110 ; 95 \% \mathrm{Cl}=-181$ to $-40 ; r^{2}=0.14 ; p<0.01$ ), the late AM increased with increasing $\mathrm{TI}$ (Supplementary figure 3-bottom-left: slope $=81 ; 95 \% \mathrm{Cl}=20$ to 142 ; $r^{2}=0.10 ; p<0.01$.

The third of cells with the lowest $\mathrm{TI}(\mathrm{N}=21)$ showed a mean positive early $\mathrm{AM}(26 \pm 6.2 \% \mathrm{p}<0.001$, Wilcoxon signed rank test) and the high transient sub-population almost no early AM $(2.7 \pm 5.7 \% p=0.38$, Wilcoxon signed rank test) (Supplementary figure 2c). Again, like in experiment 1, this relationship reversed - here even significantly - in the late response period ( $p<0.05$, Mann-Whitney test), such that the 
low-TI cells $\mathrm{AM}$ is down-regulated to $16.5 \pm 6.6 \%(p<0.01)$ and the high-Tl cells reach an AM of $29.9 \pm 5.6 \%(p<0.0001)$ and are thus significantly stronger modulated than during the early response pe$\operatorname{riod}(\mathrm{p}<0.001$, two-tailed t-test).

The attentional delay could not be determined in experiment 2, because of the skewed TIdistribution. Grouping the cells in 5 TI-bins of equal range, the upper two bins only contained data from three and one cell, respectively, and were thus to noisy for a statistically reliable analysis. However, the time-course of $A M$ in the aforementioned high- and low-transient trimester reveal a delayed increase of the high transient cells AM by about $220 \mathrm{~ms}$ after stimulus onset (Supplementary figure 2d).

a)

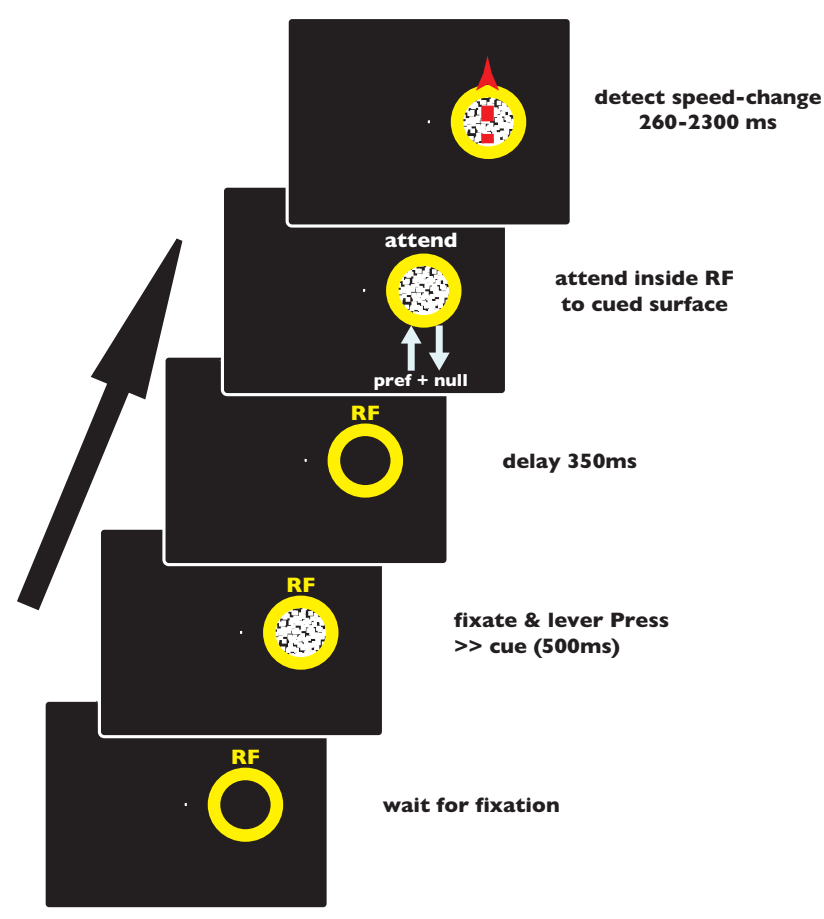

b)

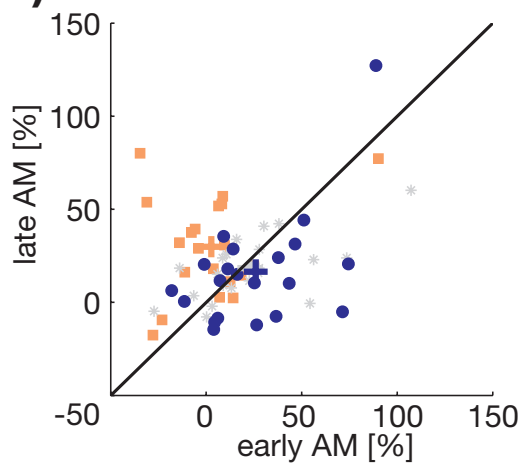

c)

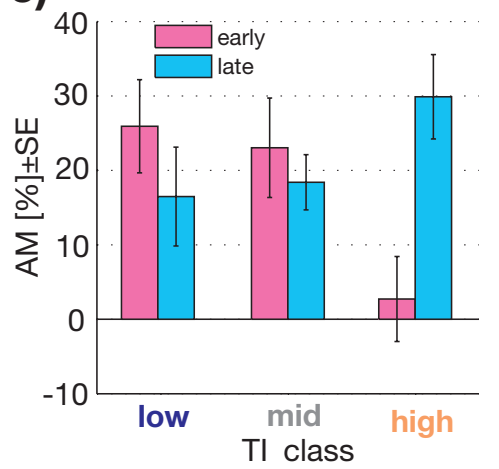

d)

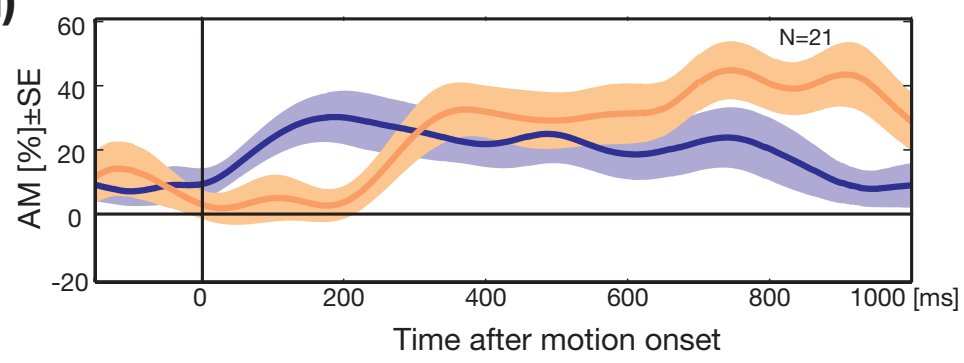

Supplementary Figure 2: Experiment 2:

a) Sequence of events in a single trial in experiment 2. When the monkey fixated and pressed he lever, a cue appeared to indicate, what surface to attend. After a brief delay of $350 \mathrm{~ms}$ the transparent motion stimulus appeared an the monkey had to indicate a speed-change of the cued surface.

b) Relationship of the individual cells 'early' and 'late' attentional modulation in experiment 2. The data-points are colored according to their membership to the respective $\mathrm{TI}$ classes (blue = low $\mathrm{TI}$ trimester; gray = middle $\mathrm{TI}$ trimester; orange = high $\mathrm{TI}$ trimester; $\mathrm{N}=63$ ).

c) Mean \pm SE attentional modulation of the high-, mid- and low-transient sub-population, either in the 'early' (magenta) or 'late' (cyan) response period.

d) Temporal dynamics of the Mean $\pm \mathrm{SE}$ attentional modulation of the high- and low-transient sub-population ( $\mathrm{N}=21$, each). While the low-transient cells further increase their positive AM immediately with motion stimulus onset, the high-transient cells behave indifferent during the $\sim 220 \mathrm{~ms}$ of their transience, thereafter their AM even overcomes that of the low-transient cells. 
PIEPER et al.: Response Saturation determines Attentional Modulation onset in MT Neurons (prep. for subm.)

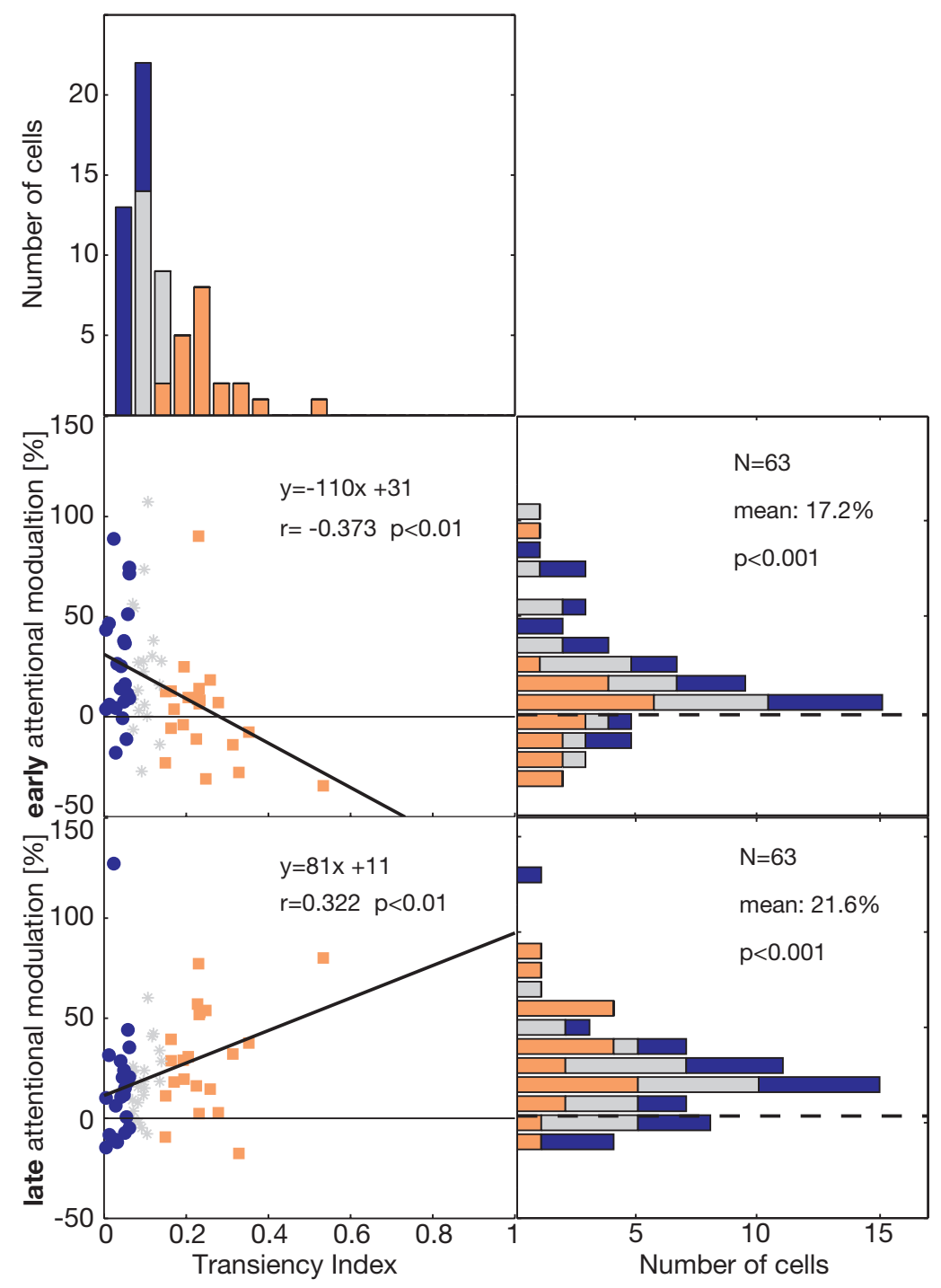

\section{Supplementary Figure 3: Experiment 2:}

Statistics of the early (middle plots) and late (lower plots) attentional modulation of 63 neurons with respect to their transiency (upper plot). The colors indicate the membership to either the low-, medium- and high-transient sub-population (blue, gray, orange) as referenced in the rest of this publication. The distribution of Tls is skewed to lower values. During the early response period the cells are at mean positively modulated ( $\varnothing: 17.2 \% ; p<0.001)$ by attention. However, the regression $(p<0.01)$ through the AM as function of the $\mathrm{TI}$ shows, that low transient cells are more likely to be positively attentional modulated while high transient cells show a different AM during this period of the trial. This relationship is reverses $(p<0.01)$ during the late, sustained response period. The mean attentional modulation slightly increases to $21.6 \%(p<0.001)$, but this now mainly caused by the strong AM of high transient cells. Moreover, the AM of the low transient cells is somehow reduced as compared to the early response period. 
54CHAPTER 3. RESPONSE SATURATION DETERMINES ATTENTIONAL MODULATION ONSET 


\section{Chapter 4}

\section{Dynamic shiftsof visual receptive fields in cortical area MT by spatial attention}

Spatial RF properties of higher visual neurons are subject to attentional modulation. Already one of the earliest single-cell studies on attention revealed that the response of a neuron is critically determined by the attended of two different stimuli within its RF (Moran and Desimone, 1985), that is, V4 neurons tended to respond as if only the attended stimulus was inside its' RF. This a finding that has been corroborated by others (Reynolds et al., 1999; Treue and Martínez-Trujillo, 1999). Moran and Desimone (1985) and Reynolds et al. (1999) hypothesized that RFs shift towards and/or shrink around the attended stimulus and by that reduce the impact of the unattended stimulus on the neurons response.

We directly tested this hypothesis in monkey area MT neurons. The monkeys performed a spatial attention task on either of three stimuli: one outside the RF, two inside the RF. All three stimuli moved in the cells 'null'-direction. Simultaneously we presented preferred stimuli at varying positions inside the RF and recorded the neurons responses during this mapping-procedure. We found that the spatial focus of attention determined the effectivity of the preferred stimuli. I.e., a mapping stimulus near the attentional focus could cause spike-response but failed to do so if the attentional focus shifted further away. This means the spatial RF significantly shifted towards the focus of attention. Also a small shrinkage of the RF was found. 



\title{
Dynamic shifts of visual receptive fields in cortical area MT by spatial attention
}

\author{
Thilo Womelsdorf ${ }^{1,2}$, Katharina Anton-Erxleben ${ }^{2}$, Florian Pieper ${ }^{2} \&$ Stefan Treue ${ }^{2}$ \\ Voluntary attention is the top-down selection process that focuses cortical processing resources on the most relevant sensory \\ information. Spatial attention-that is, selection based on stimulus position-alters neuronal responsiveness throughout primate \\ visual cortex. It has been hypothesized that it also changes receptive field profiles by shifting their centers toward attended \\ locations and by shrinking them around attended stimuli. Here we examined, at high resolution, receptive fields in cortical area \\ MT of rhesus macaque monkeys when their attention was directed to different locations within and outside these receptive fields. \\ We found a shift of receptive fields, even far from the current location of attention, accompanied by a small amount of shrinkage. \\ Thus, already in early extrastriate cortex, receptive fields are not static entities but are highly modifiable, enabling the dynamic \\ allocation of processing resources to attended locations and supporting enhanced perception within the focus of attention by \\ effectively increasing the local cortical magnification.
}

\begin{abstract}
Vision at an attended location is faster, more accurate, and of higher spatial resolution and enhanced sensitivity for fine changes ${ }^{1-4}$. Stimuli outside this 'spotlight of attention' appear to have lower contrast or might not be perceived at all ${ }^{5,6}$. Physiologically, one well-investigated effect of attention in visual cortex is a multiplicative modulation of neuronal responses ${ }^{7-9}$. But not all observed effects of attentional modulation are clearly multiplicative ${ }^{10-11}$. Most prominently, this is the case for the effect observed when one of two stimuli inside the receptive field is attended to: attending to the stimulus that elicits the stronger sensory response when presented alone typically enhances responses, whereas attending to the less optimal stimulus reduces responses ${ }^{12-14}$. It has been suggested ${ }^{12}$ that the neural basis of this differential, push-pull modulation of the respective effectiveness of each stimulus is a shrinkage of receptive fields around the attended stimuli. This would attenuate the influence of unattended stimuli at nearby locations. This influential hypothesis has never been empirically validated. Such changes in the profiles of receptive fields would have far-reaching consequences in successive areas of the cortical processing hierarchy ${ }^{15-18}$. In particular, it would provide higherorder areas with an almost exclusive representation of stimuli at the attended spatial location ${ }^{19}$.
\end{abstract}

\section{RESULTS}

Neuronal shifts with attention inside the receptive field

To investigate the influence of attention on receptive fields, we recorded from 78 neurons in cortical area MT of two macaque monkeys. Area MT is an early processing stage in the dorsal pathway and is central for the processing of visual motion information. Recordings were made while the monkeys' attention was directed to one of two stimuli (S1, S2) moving in the antipreferred direction inside the receptive field, or to a third stimulus (S3) positioned outside the receptive field (Fig. 1). We acquired high-resolution maps of a neuron's receptive field by presenting a succession of brief probe stimuli at up to 52 positions covering the receptive field (sparing the locations of S1 and S2) while the monkey's attention was allocated to one of the three stimuli. Probe stimuli were of the same size as S1, S2 and S3 but of higher contrast, and they moved in the preferred direction of the neuron. We conjectured that the hypothesized distortion of the receptive field would result in a push-pull effect, enhancing probe responses around the attended location and reducing responses to the probe farther from this focus of attention.

The results for an example neuron (Fig. 2; see also Supplementary Fig. 1 online) illustrate that the most responsive part of the receptive field was shifted substantially toward the attended position inside the receptive field (that is, when attention was directed toward S1 and S2, which were located inside the receptive field). To quantify this effect across our sample of $/ 8$ MT neurons, we determined the 'neuronal shift': the amount by which the center of mass of each receptive field shifted between the conditions when attention was directed to S1 versus S2 along the axis of the 'attentional shift' (that is, the connection between the locations of S1 and S2). Positive values indicated shifts in the same direction as the attentional shift (that is, toward the focus of attention; Fig. 3a). Across our cells, we found a highly significant neuronal shift that averaged $30.3 \%$ of the attentional shift (Fig. $3 \mathbf{b}$ horizontal axis, $P<0.001, t=14.0$, one-sample $t$-test). For the sample of receptive field sizes and stimulus locations in our study, this corresponded to an average shift of $3.0^{\circ}$ of visual angle or $22 \%$ of the receptive field diameter. Additionally, we determined the shift for the orthogonal direction (with positive values indicating shifts toward the

${ }^{1}$ F.C. Donders Centre for Cognitive Neuroimaging, Radboud University, Kapittelweg 29, 6525 EN Nijmegen, The Netherlands. ${ }^{2}$ Cognitive Neuroscience Laboratory, German Primate Center, Kellnerweg 4, 37077 Göttingen, fermany. Correspondence should he addressed to T.W. (t. Womelsdorf@fcdonders.ru.nl).

Received 7 June; accepted 20 July; published online 13 August 2006; doi:10.1038/nn1748 


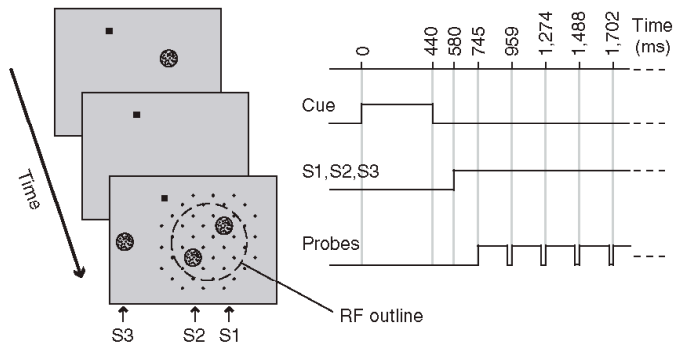

Figure 1 Experimental protocol. Time course of events and example of the placement of cue, stimuli and probes in an experimental trial (details in Methods). Black square, fixation point.

fovea) as a measure of the variability of the data. 'I'his distribution showed no significant bias (Fig. 3 b vertical axis, $P=0.48$ ).

To determine how many of the individual cells showed a significant shift, we performed a bootstrap analysis (Supplementary Note online). Of our 78 cells, $49(63 \%)$ showed a significant $(P<0.05)$ receptive field shift in the direction of the attentional shift, and none shifted significantly in the opposite direction. In contrast, the orthogonal shift was significant $(P<0.05)$ in only 3 cells $(3.8 \%)$, indicating that the receptive fields displacement fell along the axis of the attentional shift.

We can rule out the possibility that the neuronal shift was due to small differences in eye position across conditions, because we calculated the deviation in eye position along the axis of the attentional shift and found an average displacement of only $0.0182^{\circ}\left( \pm 0.0065^{\circ}\right.$, s.e.m.; details in Supplementary Note and Supplementary Fig. 2 online).

\section{Receptive field size changes with attention}

To detcrmine if the neuronal shift was accompanied by the hypothesized shrinkage of the receptive field around the attended stimulus, we compared the size of receptive fields when attention was directed inside versus outside the receptive field. Whereas receptive fields were, on average, $4.3 \%$ smaller with attention inside the receptive field (Fig. 3c), this effect was only marginally significant $( \pm 3.4 \%, 95 \%$ confidence interval, $P<0.05, t=-2.56$, paired $t$-test). Receptive field shrinkage was isotropic - that is, similar in magnitude parallel and orthogonal to the direction of the attention shift (Supplementary Note and
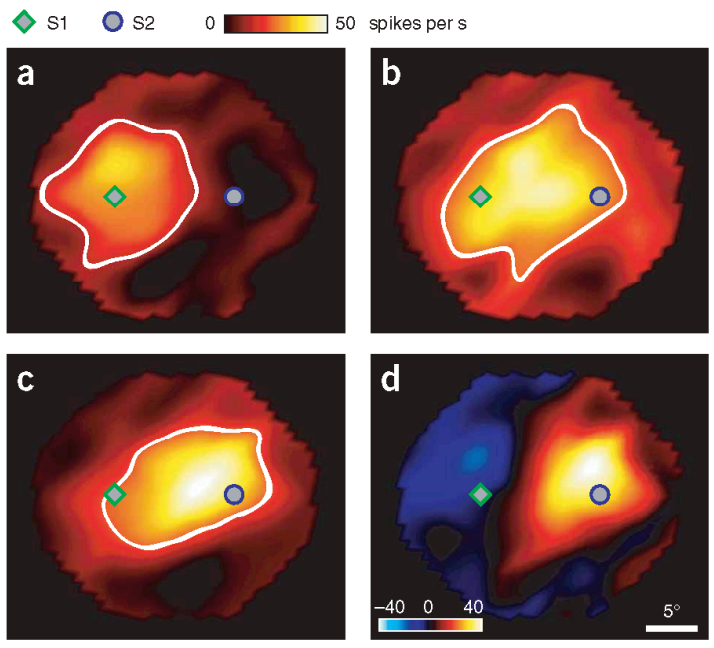

Supplementary Fig. 3 online). Thus, the influence of spatial attention was dominated by a shift, rather than a shrinkage, of receptive fields, and our observation of only a small amount of shrinkage resonates with the psychophysical observation of a coarse spatial resolution of visual attention ${ }^{20}$.

Our findings demonstrate that the enhanced/reduced response of MT neurons when spatial attention is directed to the preferred/ antipreferred of two stimuli inside their receptive field can be accounted for by a systematic and large change in the receptive field profile. In effect, attention changes the spatial filtering characteristics of those MT neurons whose receptive fields overlap with the currently attended location.

\section{Spatial extent of receptive field shifts with attention}

For an attentional location just outside the receptive field, a previous study in area $V 4$ (ref. 21) has demonstrated that receptive fields are distorted toward the location of attention. We were wondering if such an effect is also present in area MT and if it extends beyond the immediate surround of the receptive field. To investigate this issue, we compared the receptive field profile when attention was directed to either S1 or S2, located inside the receptive field (the 'in' condition), to the profile when attention was directed to S3, located far outside the receptive field in the opposite hemifield (the 'out' condition; Fig. 4a). If the receptive field in the out condition was unaffected by the location of the attentional focus relative to the receptive ficld, the in condition should shift the receptive field center along a vector pointing directly at the attended stimulus' location inside the receptive field. If, on the other hand, the receptive field center in the out condition was already attracted toward the attended stimulus' location outside the receptive field, then switching to the in condition should not only shift the center to the new location of attention, but should also release it from the attraction toward the $\mathrm{S} 3$ location. In this casc, the resulting shift vector should not point directly at the attended stimulus' location inside the receptive field, but should be deviated somewhat by a vector component pointing away from the direction of $S 3$. We found just that for a significant majority of the vectors $(62.5 \%, P<0.05$, Wilcoxon signed rank test; Fig. 4b). Further support for a shift of the receptive field center toward the location of attention in the out condition came from the finding that the eccentricitics of the receptive ficlds in trials with attention outside the receptive field were smaller than their eccentricities in trials with attention inside the receptive field $(7.9 \%, P=0.019$, paired $t$-test). These findings are not only in agreement with the findings from $\mathrm{V} 4$, but go well beyond them in demonstrating a far-reaching effect of spatial attention that even affects neurons with receptive fields in the opposite hemifield.

\section{DISCUSSION}

One effect of spatial attention that has been reported frequently is a multiplicative modulation of tuning curves ${ }^{7-9}$. The push-pull modulation of MT receptive fields with shifts of spatial attention within the

Figure 2 Receptive field profiles of an example cell, as 2D surface plots. (a c) Receptive field (RF) profiles when attention was directed inside the RF, to stimulus S1 (a) or S2 (c), or when attention was directed outside the RF, to S3 (b). The surface color at each point in the plots indicates the increase in the neuron's response elicited by the presentation of a probe stimulus at that position, over the response observed in the absence of a probe (that is, when only $\mathrm{S} 1$ and $\mathrm{S} 2$ were present). Supplementary Fig. 1 online shows the same data as absolute firing rates. (d) Difference map, computed by subtracting the RF when attention was on S1 from the RF when attention was on S2. The map illustrates that shifting attention from $S 1$ to $S 2$ enhances responsiveness around $\mathrm{S} 2$ and reduces it near $\mathrm{S} 1$. 


\section{ARTICLES}

Figure 3 Quantification of RF shift and shrinkage. (a) Convention used to quantify the shift of RF centers. (b) Magnitudes of the neural shifts, along the axis of the attentional shift ( $x$-axis and top histogram) or orthogonal to it ( $y$-axis and right histogram; positive values indicate shifts toward the fovea). Light gray, monkey $D$; dark gray, monkey R. Circle, example cell illustrated in Figure 2 (attentional shift: $+53.6 \%$.). Filled and unfilled histogram bars and symbols indicate significant $(P<0.05)$ and nonsignificant shifts, respectively. The top histogram shows a highly significant mean neuronal shift toward the attended stimulus $(30.3 \% \pm 4.3 \%(95 \% \mathrm{Cl})$, $P<0.001)$. This mean shift was larger in monkey $\mathrm{R}(35 \% \pm 4.8 \%)$ than in monkey $\mathrm{D}(18 \% \pm 6.2 \%)$. There was no significant bias in the direction orthogonal to the direction of the attentional shift (mean: $1.3 \%+3.5 \%$ ). (c) Histogram of RF size changes when attention was direcled oulside versus inside Lhe RF. Slars,

a

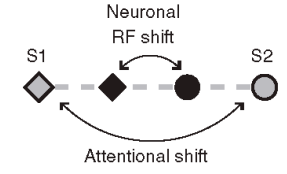

C

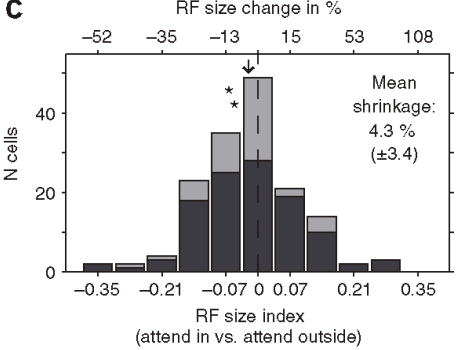

b

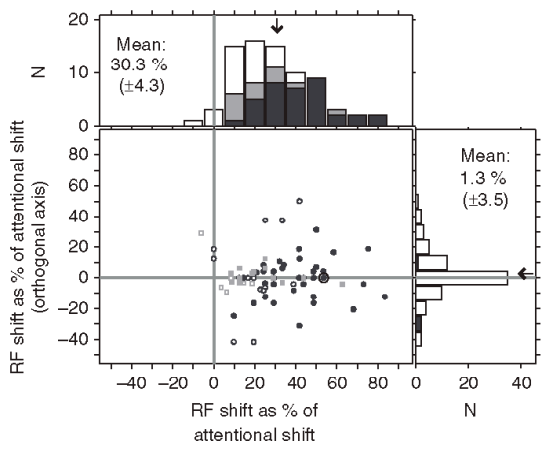

size change of the example cell from Figure 2; the RF shrank when attention was directed toward S1 or S2 $(-9.5 \%$ and $-4.4 \%$, respectively). The histogram is shifted slightly to the left, indicating a small but significant mean reduction of RF size $(4.3 \pm 3.4 \%, 95 \% \mathrm{Cl}, P<0.05$, paired $t$-test) when attention was directed into the RF. The size reduction did not differ significantly between the two monkeys and was around the 0.05 significance level when the two data sets were analyzed separately (monkey $\mathrm{R}:-3.7 \% \pm 4.3 \%, P=0.077$; monkey D: $-5.7 \% \pm 5.6 \%, P=0.039$ ).

receptive field is not a multiplicative change of a neuron's spatial tuning curve; nevertheless, it is important to point out that the underlying attentional modulation might still be multiplicative. If attention differentially acts on the neurons with smaller receptive fields that provide the input to MT, the observed modulation in receptive field profiles could be achieved with multiplicative effects: for instance, by increasing the response gain of input neurons representing the attended location and decreasing the response gain of the neurons representing unattended regions within the MT receptive field ${ }^{15}$.

Our findings show that spatial attention shifts the receptive fields of MT neurons toward the attentional focus. Such a dynamic routing provides a powerful mechanism to increase selectivity of visual representations within and across functionally specialized visual areas, and serves to constrain models of the perceptual organization of selective visual processing. For the neural population as a whole, the spatial shift reflects the additional recruitment of processing resources at the focus of attention. Paralleling the increase in the observed magnitude of attentional modulation, the shifts of receptive fields probably increase with increasing receptive field size in successive areas of the visual hierarchy. It could also be the neural correlate of various perceptual effects that are centered on the focus of attention, including enhanced processing accuracy and spatial resolution close to the attentional focus, suppression in its surround and distortions in spatial judgments ${ }^{22-25}$.

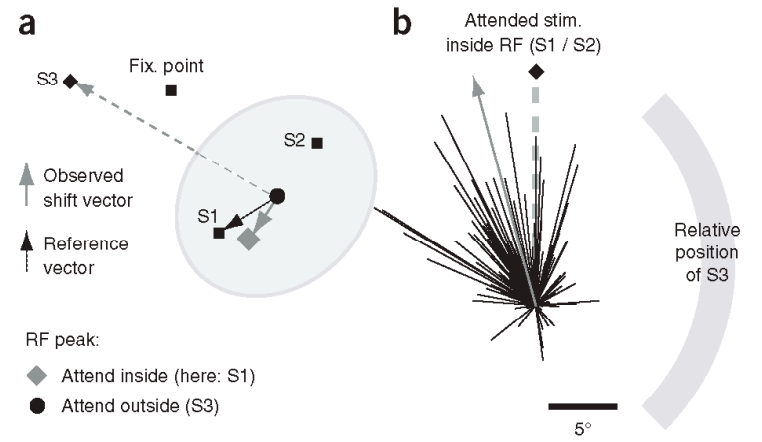

In summary, our finding that receptive fields are highly malleable by the attentional state demonstrates a dynamic spatial filtering system that could provide the neuronal correlate of the central purpose of attentional modulation: namely, the allocation of processing resources to the attended stimuli at the expense of the unattended ones. This dynamic modification by spatial attention seems to affect the receptive field mosaic across the whole visual field and is likely to be part of a mechanism active during the planning or execution of eye movement ${ }^{26-29}$. Furthermore, the similarity between the far-reaching influence of spatial attention across the visual field and the distribution of feature-based attention ${ }^{7}$ supports the hypothesis that both rely on a common underlying mechanism.

\section{METHODS}

Electrophysiological recording. All procedures reported in this study were approved by the district government of Braunschwcig, Lower Saxony, Germany. Neuronal activity was recorded from 57 and 21 single isolated cells from

Figure 4 Receptive field shift when attention is directed inside versus outside the receplive field. (a) Typical slimulus anrangerrent. Black circle and gray diamond, RF center positions. Vectors indicate the direction of a direct shift of the RF center toward the attended stimul us inside the RF (the 'reference vector') and the direction of the observed shift. Note that the eccentricity of the RF when attention was directed inside the RF (distance from fixation point to gray square) was, on average, $7.9 \%$ larger than the RF eccentricity when attention was directed to 33 (distance from fixation point to black circle). We analyzed the data from the two monkeys separately and found that this effect was significant only in monkey $\mathrm{R}(9.9 \%, F-0.02)$. (b) Distribution of observed shift vectors. These data are based on the 64 cells for which sufficient data were available for both the attend-outside (to stimulus S3) and each of the attend-inside (to stimulus S1 or S2) conditions. All vectors were rotated, such that the reference vector would point straight up, and flipped horizontally (if necessary), such that the \$3 stimulus position would be on the right. The gray arrow pointing in the direction opposite to the location of $\mathrm{S} 3$ indicates a systematic and significant deviation of the vectors (average deviation of $15.2^{\circ}, 95 \%$ angular confidence: $\pm 9.8^{\circ}, P<0.05$ ), and implies a far-reaching modulation of receptive fields by attention. We analyzed the data from the two monkeys separately and found that this effect was significant only in monkey $R\left(19.2 \pm 10.5^{\circ}, P<0.05\right)$. 
monkeys $R$ and $D$, respectively, with tungsten electrodes (impedance 1.0-4.0 M $\Omega$, Frederick Haer). Cell isolation was based on window discrimination (BAK Electronics or Plexon). Cells were localized in area MT by their physiological characteristics and the histological reconstruction of recording sites in monkey R. Access to MT was provided by a craniotomy and a recording chamber surgically implanted above the superior temporal sulcus of the lef hemisphere. During the experinnent, a custom computer program running on an Apple Macintosh PowerPC controlled stimulus presentation, and monitored and recorded eye positions and neuronal and behavioral responses. Eye positions were determined using a high-resolution, video-based eye tracking system (ET49, Thomas Recording GmbH) with a sampling frequency of 230 $\mathrm{Hz}$, and were digitized and stored at $200 \mathrm{~Hz}$.

Visual stimuli. Stimuli were moving random dot patterns (RDPs) of smal bright dots (density: 10 dots per deg $^{2}$ ) plotted within a stationary circular aperture on a dark $(0.7 \mathrm{~cd})$ computer monitor. For each receptive field, stimuli S1 and S2 were placed at similarly responsive positions in the receptive field at equal eccentricity and equidistant from the center of the receptive field when the monkey was directing its attention to the fixation point. $\mathrm{S} 3$ was placed in the opposite hemifield. Initial estimation of this 'sensory' receptive field center was based on a manual mapping with a mouse-controlled RDP and with quantitative mapping during the main experiment. Note that the shift and shrinkage of the receptive field when attention was directed to S1 or S2 could cause the other stimulus to fall outside the receptive field on those trials (see Fig. $2 a-c$ for an example). Note also that the initial manual mapping was used only for the placement of the stimuli. All receptive fields profiles used in the analysis were mapped quantitatively during temporally interleaved trials in which attention was directed to S1, S2, S3 or the fixation point. Therefore all receptive field profiles used in the analysis were determined with temporally interleaved mapping. This ensured that the results were not contaminated by potential changes in a neuron's isolation or responsiveness over time.

Stimuli S1, S2 and S3 moved in the cell's antipreferred direction and with a reduced luminance $(19 \mathrm{~cd})$ in order to prevent a saturation of the cell's response due to the presence of $\mathrm{S} 1$ and $\mathrm{S} 2$ alone. A fourth RDP (the 'probe', $47 \mathrm{~cd}$ ) moving in the preferred direction of the neuron was used to probe the spatial sensitivity. This probe stimulus was of the same size as S1, S2, and S3 and was presented at the intersections of a dense grid (between 42 and 52 positions) spanning the classical receptive field and its immediate surround, but sparing the S1 and S2 location (in order to avoid potential nonlinear interactions and changes in the sensory quality of $S 1$ and $S$. that conld affect the attentional task performed on these stimuli). The longer axis of the elliptical probe grid was always along the $\mathrm{S} 1-\mathrm{S} 2$ axis.

Experimental procedure. First we isolated a single cell and determined it preferred direction. Then we centered the virtual grid of the array of probe positions at the estimated center of the receptive field (Fig. 1), in an approach similar to that used in a previous study ${ }^{30}$. A trial started once the monkey's gaze was directed within $0.75^{\wedge}$ of the fixation point. After the monkey touched a lever, the cue (a stationary RDP) appeared for $445 \mathrm{~ms}$ at the upcoming position of either S1, S2 or S3, indicating the 'target' location for the trial. After a 145-ms interstimultus interval, S1, S2 and S3 were presented. The task was to detect a brief $(80 \mathrm{~ms}$ ) phase during which the target (the stimulus at the previously cued location) moved in a different dircction, whilc ignoring cquivalent phases in the two other RDPs (the 'distracters'). Successive presentation of the receptive field probe stimulus began $160 \mathrm{~ms}$ after the onset of S1, S2 and S3. Probe duration was $187 \mathrm{~ms}$ with an interprobe blank period of $27 \mathrm{~ms}$. The direction of the target and the distracter stimuli changed $670-4,670 \mathrm{~ms}$ after the trial began; the times of these changes were randomly picked from a uniform distribution. In control trials, the monkey had to detect a change in the color of the fixation square. Trials were aborted if the monkey's gaze left the fixation window or if the monkey released the lever outside a 150 - to 750 - $\mathrm{ms}$ time window after the change of the target stimulus (for example, because the monkey released the lever in response to a distracter change, or if it failed to detect the target change).

Data analysis. To analyze the data, we used the mean neuronal responses to probe presentations from only the correctly completed trials in the three experimental conditions (that is, when attention was directed to S1 ('attend- inside' receptive field), to $\$ 2$ ('attend-inside' receptive field) or to $\$ 3$ ('attendoutside' receptive field)). The mean firing rate was computed for a 60 - to 200-ms interval after the onset of the probe stimulus. For each condition, some of the probe presentations were skipped (that is, no probe was shown) in order to determine the cell's response to $\$ 1$ and $\$ 2$ alone. This baseline was subtracted from all probe responses before the interpolation of the receptive field profile by cubic spline interpolation. These two-dimensional (2D) profiles were used to determine receptive field centers and sizes.

We calculated the shift of the receptive field (Fig. $3 \mathbf{b}$ ) between attentional conditions by using the center of mass of one-dimensional projections of the receptive field surface. For this analysis, we averaged the activity of the receptive field profile orthogonal to the axis connecting the two stimuli within the receptive field (Fig. 3b, horizontal axis) or to an orthogonal axis (Fig. $\mathbf{3 b}$ vertical axis). Averaging was limited to regions of the receptive field surface that exceeded two s.d. of the baseline response in any of the attentional conditions, in order to exclude visual field regions that did not contribute to the receptive field profile. For each $2 \mathrm{D}$ projected receptive field slice, we determined the center of mass and the peak positions (data not shown but qualitatively identical to results with the center of mass). Receptive field shifts between the two attend-inside conditions are expressed as the proportional distance of the center of mass relative to the reference distance between the stimuli S1 and S2 (Fig. 3a). Shift values were positive when the receptive field center lay closer to the attended stimulus in the respective attentional conditions.

To quantify the statistical significance of the shift of the neuronal receptive fields for individual cells, we applied a bootstrap method (details in Supplementary Note).

The receptive field size was calculated as the square root of the area in which the $2 \mathrm{D}$ receptive field surface exceeded the half-maximal response (after subtracting the baseline responses-that is, responses in the same attentional condition when $\mathrm{S} 1$ and $\mathrm{S} 2$ were present but no probe stimulus was shown). We compared receptive field sizes when attention was directed to the stimulus outside the receptive field versus when attention was directed to either of the stimuli inside the receptive field, by using an attentional modulation index ${ }^{13}$. $\left(S_{\text {in }}-S_{\text {out }}\right) /\left(S_{\text {in }}+S_{\text {out }}\right)$, where $S_{\text {in }}$ and $S_{\text {out }}$ are the size of the receptive field when attention was directed inside and outside, respectively. The size index ranges between -1 and 1 ; negative values reflect a smaller receptive field size when attention was directed inside the receptive field compared to outside the receptive field (Fig. $3 \mathbf{c}$ ). The average index value is a conservative estimate as it corresponds to a geometric mean (that is, the mean is less influenced by large values than a regular mean wowld he)

Note: Supplementary information is available on the Nature Neuroscience website.

\section{ACKNOWLEDGMENTS}

We thank T. Tzvetanov and M. Husain for discussions in the course of this study; U. Grosshennig, D. Prüsse, K. Fahrner and R. Rode-Brockhausen for technical assistance; and D. Heller-Schmerold and S. Stuber for administrative assistance. Thi work was supported by the German Research Foundation (SFB 550 \& GRK 289) and the Bernstein Center for Computational Neuroscience, Göttingen (Federal Ministry of Education and Research grant 01GQ0433).

\section{AUTHOR CONTRIBUTIONS}

T.W. and S.T. conceived the experiment and performed the data analysis. T.W. and K.A.-F. performed the experiments. F.P. provided technical assistance and helped during the experiment. T.W. and S.T. wrote the paper.

COMPETING INTERESTS STATEMENT

The authors declare that they have no competing financial interests.

Published online at htto://www.neture.com/naturencuroscience

Reprints and permissions information is available online at http://npg.nature.com/ reprintsandpermissions!

1. Yeshurun, Y. \& Carrasco, M. Attention improves or impairs visual performance by enhancing spatial resolution. Nature 396, 72-75 (1998).

2. He, S., Cavanagh, P. \& Intriligator, J. Attentional resolution and the locus of visua awareness. Nature 383, 334-337 (1996)

3. Hawkins, H.L. et al. Visual attention modulates signal detectability. J. Exp. Psychol Hum. Percept. Perform. 16, 802-811 (1990). 


\section{ARTICLES}

4. Carrasco, M., Williams, P.E. \& Yeshurun, Y. Covert attention increases spatial resolution with or without masks: support for signal enhancement. J. Vis. 2, 467-479 (2002).

5. Carrasco, M., Ling, S. \& Read, S. Attention alters appearance. Nat. Neurosci. 7 308-313 (2004).

6. Simons, D.J. \& Rensink, R.A. Change blindness: past, present, and future. Trends Cagn. Sci. 9, 16-20 (2005)

7. Treue, S. \& Martinez Trujillo, J.C. Feature-based attention influences motion processing gain in macaque visual cortex. Nature 399, 575-579 (1999).

8. McAdams, C.J. \& Maunsell, J.H. Effects of attention on orientation-tuning functions of single neurons in macaque cortical area V4. J. Neurosci. 19, 431-441 (1999).

9. Martinez-Trujillo, J.C. \& Treue, S. Feature-based attention increases the selectivity of population responses in primate visual cortex. Curr. Biol. 14, 744-751 (2004).

10. Reynolds, J.H. \& Chelazzi L. Attentional modulation of visual processing. Annu. Rev. Neurosci. 27, 611-647 (2004)

11. Treue 5 . Neural correlates of attention in primate visual cortex. Trends Neurosci. 24, 295-300 (2001)

12. Moran, J. \& Desimone, R. Selective attention gates visual processing in the extrastriate cortex. Science 229, 782-784 (1985)

13. Treue, S. \& Maunsel I, J.H. Effects of attention on the processing of motion in macaque midde temporal and medial superior temporal visual cortical areas. $J$. Neurosci. 19, 7591-7602 (1999)

14. Reynolds, J.H., Chelazzi, L. \& Desimone, R. Competitive mechanisms subserve attention in macaque areas V2 and V4. J. Neurosci. 19, 1736-1753 (1999).

15. Maunsel, J.H.R. \& McAdams, C.J. Effects of attention on the responsiveness and selectivity of individual neurons in visual cerebral cortex. in Visual Attention and Cortical Circuits (eds. Braun, J., Koch, C. \& Davis, J.L.) Ch. 6 103-120 (MIT Press, Cambridge Massachusetts, 2001).

16. Reynolds, J.H. \& Desimone, R. The role of neural mechan isms of attention in solving the binding problem. Neuron 24, 19-29 (1999).

17. Salinas, E. \& Abbott, L.F. A model of multiplicative neural responses in parietal cortex. Proc. Natl. Acad. Sci. USA 93, 11956-11961 (1996)
18. Itti, L. \& Koch, C. Computational modelling of visual attention. Nat. Rev. Neurosci. 2, 194-203 (2001).

19. Everling, S., Tinsley, C., Gaffan, D. \& Duncan, J. Filtering of neural signals by focused attention in the monkey prefrontal cortex. Nat. Neurosci, 5, 671-676 (2002)

20. Intriligator, J. \& Cavanagh, P. The spatial resolution of visual attention. Cognit. Psychol. 43, 171-216 (2001)

21. Connor, C.E., Preddie, D.C., Gallant J. L. \& Van Essen, D.C. Spatial attention effects in macaque area V4. J. Neurosci. 17, 3201-3214 (1997)

22. Suzuk, S. \& Cavanagh, P. Focussed attention distorts visual space: an attentiona repulsion effect. J. Exp. Psychol. Hum. Percept. Perform. 23, 443-463 (1997).

23. LaBerge, D., Carlson, R.L., Williams, J.K. \& Bunney, B.G. Shifting attention in visua space: tests of moving-spotlight models versus an activity-distribution model. J. Exp. Psychol. Hum. Percept. Perform. 23, 1380-1392 (1997).

24. Müsseler, J Stork, S \& Kerzel, D. Comparing mislocalizations with moving stimuli. The Fröhlich effect, the flash-lag effect and representational momentum. Vis. cogn. 9 $120-138$ (2002).

25. Tsal, Y. \& Bareket, T. [ffects of attention on localization of stimuli in the visual field Psychon. Bull. Rev. 6, 292-296 (1999)

26. Ben Hamed, S., Duhamel, J.R., Bremmer, F. \& Graf, W. Visual receptive field modulation in the lateral intraprietal area during attentive fixation and free gaze. Cereb. Cortex $12,234-245$ (2002).

27. Duhamel, J.R., Colby, C.L. \& Goldberg, M.E. The updating of the representation of visual space in parietal cortex by intended eve movements. Science 255, 90-92 (1992)

28. Tol ias, A.S. et al. Eye movements modulate visual receptive fields of V4 neurons. Neuron 29, 757-767 (2001)

29. Krekelberg, B., Kubischik, M., Hoffmann, K.P. \& Bremmer, F. Neural correlates of visua localisation and perisaccadic mislocalisation. Neuron 37, 537-545 (2003)

30. Britten, K.H. \& Heuer, H.W. Spatial surnmation in the receptive fields of MT neurons. J. Neurosci. 19, 5074-5084 (1999). 


\title{
Supplementary Information for
}

\author{
Dynamic shifts of visual receptive fields \\ in MT by spatial attention
}

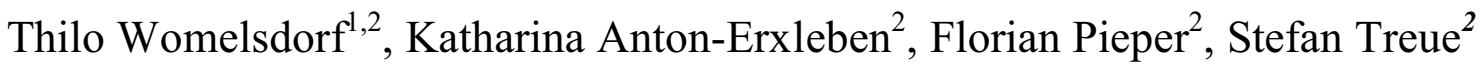 \\ ${ }^{1}$ F.C. Donders Centre for Cognitive Neuroimaging, Radboud University, Kapittelweg 29, 6525 EN Nijmegen, \\ The Netherlands \\ ${ }^{2}$ Cognitive Neuroscience Laboratory, German Primate Center, Kellnerweg 4, 37077 Göttingen, Germany
}

The supplementary material provides additional information about three results:

- We address the possibility that the attentional shift effects observed were caused by changes in eye position.

- We provide information about the bootstrap method used to estimate the statistical reliability of the receptive field centre shift in individual neurons.

- We illustrate that the moderate shrinkage of receptive fields in conditions with attention inside versus outside the receptive field is isotropic, i.e. it is not biased with regard to the direction of attention.

The receptive field shift cannot be explained by changes of the eye position within the fixation window

Receptive fields in area MT are retinotopic, i.e. they move across the visual field with changes in eye position. It is therefore conceivable that the shifts in receptive field centres we have observed are due to systematic differences in eye positions between the attentional conditions. Such a shift would only be able to provide a small contribution to the changes we have observed since the average shift of receptive field centres was $3.0^{\circ}$ (see main text, Fig. $3)$, much larger than the diameter of the fixation window $\left(1.5^{\circ}\right)$ that the monkey had to stay within throughout all trials. Nevertheless, we computed the difference of the average eye position in conditions with attention to either stimulus S1 or stimulus S2 in the direction from $\mathrm{S} 1$ to $\mathrm{S} 2$, i.e. the axis along which attention was shifted between conditions. This analysis revealed a very small difference in average eye position of only $0.018^{\circ}\left( \pm 0.0065^{\circ} \mathrm{SE}\right)$ between the conditions. We also calculated the average eye position difference between the 
attend-inside conditions and the attend-outside condition along the axis of the respective stimuli (i.e. along the axis from S1-to-S3 or from S2-to-S3). Similar to the previous analysis we found a very small difference of eye positions of only $0.009^{\circ}\left( \pm 0.004^{\circ} \mathrm{SE}\right)$ in the direction of the attended stimulus. Supplementary Figure 2 illustrates the distribution of gaze positions for three representative example cells in conditions with attention to either stimulus S1 (upper panels), or to S2 (lower panels) inside the receptive field. The centre of the coordinate system is the fixation point and the relative direction of the attended (nonattended) stimulus is indicated by the square (circle) plotted on the axes border. For these examples, the average difference of gaze positions along the S1-to-S2 axis ranged from $-0.036^{\circ}$ to $0.054^{\circ}$. The receptive field shifts of these conditions were $1.8^{\circ}$ to $4.2^{\circ}$ in the direction of the attended stimulus, many times larger than the differences in average gaze position. These results demonstrate that the contribution of eye position artefacts to the estimated receptive field shifts was marginal at best.

\section{Calculation of the statistical significance of the receptive field shift in individual cells}

To quantify the statistical significance of the shift of the neuronal receptive fields for individual cells we applied a bootstrap method. To this end we computed the variability of the receptive field profile by simulating our experiment 1000 times. On each repetition we simulated the response for each probe position by drawing (with replacement) and averaging across as many individual responses as were collected in the actual experiment from the original distribution of responses. To compare the receptive field centers for the two attention conditions (attend S1 versus attend S2) statistically we determined the $95 \%$ range of receptive field centers for each condition (by determining the $2.5 \%$ leftmost and $2.5 \%$ rightmost center position from the 1000 simulations). When the $95 \%$ distributions of both conditions did not overlap the difference in receptive field center positions were considered statistically significant. This procedure corresponds to a two-tailed randomization test with a significance level of $\mathrm{P}=0.05$.

\section{Spatial isotropy of changes in receptive field size with attention inside the receptive field}

We found only moderate receptive field shrinkage with attention to a stimulus inside compared to attention outside the receptive field (see Fig. 3c). On average receptive fields were $4.3 \%$ smaller when attention was directed inside the receptive field with a 95\% confidence range of $\pm 3.4 \%$. To determine whether this change in receptive field size was 
different in the direction of the attention shift (see Fig. 3a and Fig. 4) we computed the maximum extent of the receptive field parallel and orthogonal to the axis of the attentional shift, i.e. the axis from stimulus S3 outside the receptive field (the attended stimulus in the outside condition) toward stimulus S1 / S2 in conditions with attention inside the receptive field. This analysis showed no spatial bias of receptive field size changes parallel and orthogonal to the direction of the attentional shift (paired $\mathrm{t}$-test, $\mathrm{P}=0.444, \mathrm{t}=0.77$ ). The distribution of receptive field size changes parallel to the attentional shift (along the $\mathrm{x}$-axis) and orthogonal to the axis of the attentional shift (along the y-axis) are shown in Supplementary Figure 3. 

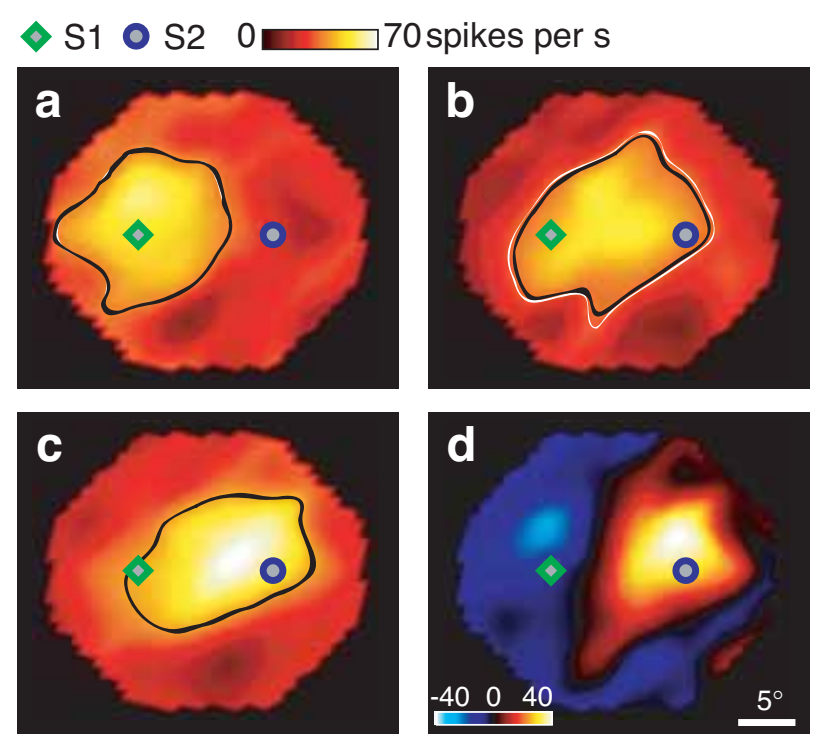

Supplementary Figure 1. Receptive field profiles of an example cell as 2dimensional surface plots (same format as in Fig. 2). The RF profiles are from the same example cell as shown in (Fig. 2). Here the plots are based on the raw response evoked during probe presentation, without subtracting the response evoked by S1 and $\mathrm{S} 2$ alone. The black outlines represent the area above half-maximum response and the white outline is based on the data in (Fig. 2).

\section{Womelsdorf, Anton-Erxleben, Pieper \& Treue Supplementary Fig. 1}




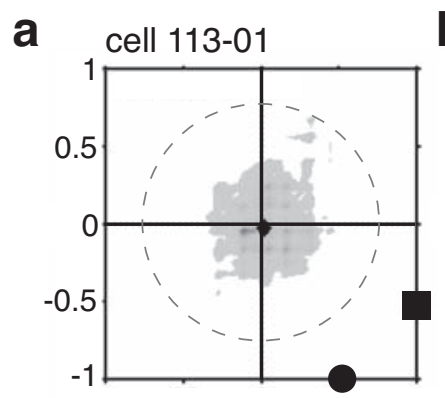

b cell 060-02

C cell 114-01
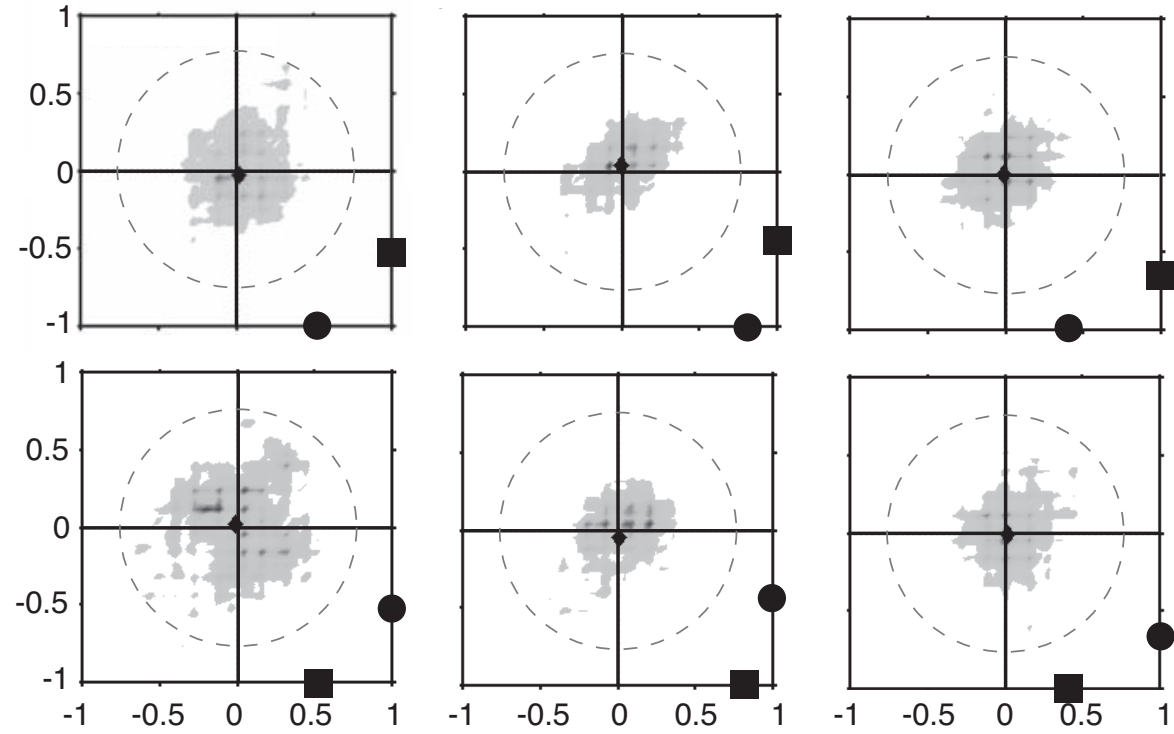

mean diff. on

S1-S2 axis :

$-0.036^{\circ}$
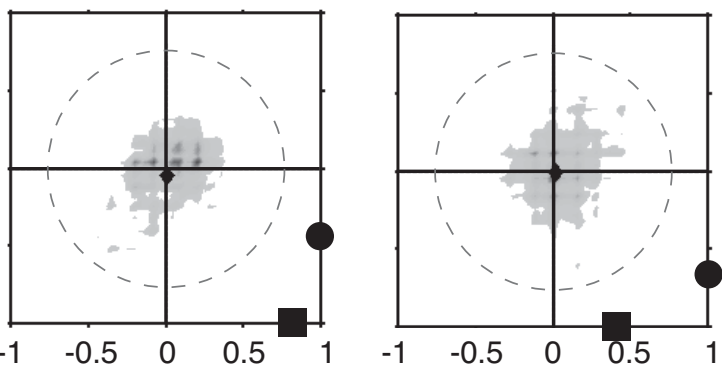

RF shift (\%):

$$
\begin{gathered}
4.2^{\circ} \\
(29.2 \%)
\end{gathered}
$$

$0.054^{\circ}$

$-0.026^{\circ}$

$1.8^{\circ}$
$(25.0 \%)$

$1.9^{\circ}$

$(14.6 \%)$

Supplementary Figure 2. Distribution of eye positions for trials where attention was directed onto one of the stimuli inside the receptive field in three example cells (a-c) The grey values represent the frequency of gaze positions relative to the fixation square represented by the central intersection. The relative direction from the fixation point to the attended (non-attended) stimulus is shown as a square (circle) on the axis border (see text for details). Axes values represent the distance of eye position from the fixation point in degrees of visual angle. The circles around the plot centres represent the virtual fixation window within which the monkey's gaze had to stay throughout each trial.

\section{Womelsdorf, Anton-Erxleben, Pieper \& Treue Supplementary Fig. 2}




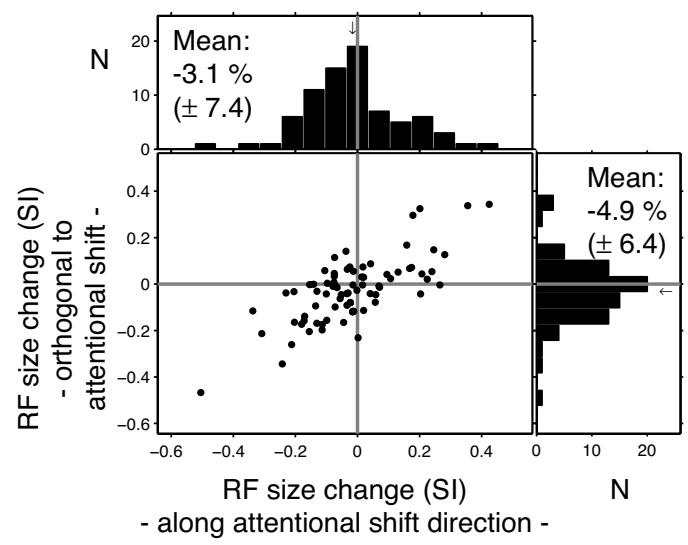

Supplementary Figure 3. Spatial isotropy of changes in receptive field size with attention inside (to stimulus S1 or S2) versus attention outside the receptive field (to stimulus S3). Shown is the distribution of receptive field size changes parallel (along the $\mathrm{x}$-axis) and orthogonal (along the $\mathrm{y}$-axis) to the axis from stimulus $\mathrm{S} 3$ to stimulus $\mathrm{S} 1 / \mathrm{S} 2$ for conditions with attention to S3 and attention to S1/S2. The receptive field size change was not statistically different parallel versus orthogonal to the axis of the attention shift (3.1\% versus $4.9 \%$ respectively).

\section{Womelsdorf, Anton-Erxleben, Pieper \& Treue Supplementary Fig. 3}


68CHAPTER 4. DYNAMIC SHIFTS OF VISUAL RECEPTIVE FIELDS IN CORTICAL AREA MT BY 


\section{Chapter 5}

\section{Summary}

The presented papers further corroborate, that visual perception perception is critically influenced by both, the short-term history of the visual input and the cognitive control of the subject. As much as perception is variable from the subjects point of view, the spatio-temporal scope of a neuron in its feature dimension is determined by a fast, reversible plasticity. Attention is a mechanism that roughly instantaneously changes computational properties of neurons if needed. We have shown that adaptation also acts on a short time scale, such that it also determines our natural viewing. Or results ultimately should engage researchers to focus on short-term - in the range of a second and below - temporal dynamics of sensory and cognitive processes and their representation in different cell-types. This may elucidate the cascade of single adaptive mechanisms that are usually overlooked in data that is averaged across seconds or minutes and inhomogeneous cell populations.

As mentioned in the preface, reducing perception to a simple sensory (de-) coding task might lead us to the wrong questions in the future. The ultimate reason for an individual's brain-anatomy and -function depends on the history of its (and its ancestors) self-environment-interaction, or decisions (see Glimcher, 2002)! Actual decisions may be seen as the result of a bayesian inference about the future selfenvironment-interaction, given the history of interactions, sensory input and evoked responses on either level of life's adaptiveness. These levels have different time constants and mechanistic background and are termed: genomic or behavioral im-

printing, learning, priming, facilitation, sensitization and 'adaptation'. The ability to direct attention is hence just another implementation of a plastic, conditional recalibration of i.e. the sensory representation that helps to come to adaptive decisions. 


\section{Bibliography}

Akiyoshi, K. (2007). Tilt illusions after oyama (1960): A review. Japanese Psychological Research, 49(1):7-19.

Alais, D. and Blake, R. (1999). Neural strength of visual attention gauged by motion adaptation. Nat Neurosci, 2:1015-1018.

Albrecht, D. G., Farrar, S. B., and Hamilton, D. B. (1984). Spatial contrast adaptation characteristics of neurons recorded in the cat's visual cortex. Journal of Physiology, 347:743-739.

Albrecht, D. G. and Hamilton, D. B. (1982). Striate cortex of monkey and cat: contrast response function. Journal of Neurophysiology, 48(1):217-237.

Albright, T. D., Desimone, R., and Gross, C. G. (1984). Columnar organization of directionally selective cells in visual area MT of the macaque. Journal of Neurophysiology, 51(1):16-31.

Allman, J. and Kaas, J. (1976). Representation of the visual field on the medical wall of occipital-parietal cortex in the owl monkey. Science, 191(4227):572-575.

Anton-Erxleben, K., Henrich, C., and Treue, S. (2007). Attention changes perceived size of moving visual patterns. Journal of Vision, 7(11):1-9.

Bair, W., Cavanaugh, J. R., Smith, M. A., and Movshon, J. A. (2002). The timing of response onset and offset in macaque visual neurons. Journal of Neuroscience, 22(8):3189-3205.

Barlow, H. and Földiak, P. (1989). Adaption and Decorrelation in the Cortex, pages 54-72. Addison-Wesley Longman Publishing Co., Inc. 
Barlow, H. B. (1961). Possible principles underlying the transformations of sensory messages. In Rosenblith, W., editor, Sensory Communication, pages 217-234. MIT Press.

Bisley, J. W. and Goldberg, M. E. (2003). Neuronal activity in the lateral intraparietal area and spatial attention. Science, 299(5603):81-86.

Blaser, E., Pylyshyn, Z. W., and Holcombe, A. O. (2000). Tracking an object through feature space. Nature, 408:196-199.

Boynton, G. M. and Finney, E. M. (2003). Orientation-specific adaptation in human visual cortex. Journal of Neuroscience, 23:8781-8787.

Bratschelet, E. (1981). Circular statistics in biology. Academic Press.

Brenner, N., Bialek, W., and de Ruyter van Steveninck, R. (2000). Adaptive rescaling maximizes information transmission. Neuron, 26:695-702.

Britten, K., Shadlen, M., Newsome, W., and Movshon, J. (1992). The analysis of visual motion: a comparison of neuronal and psychophysical performance. $J$. Neurosci., 12(12):4745-4765.

Britten, K. H. and Heuer, H. W. (1999). Spatial summation in the receptive fields of MT neurons. Journal of Neuroscience, 19(12):5074-5084.

Brodmann, K. (1909). Vergleichende Lokalisationslehre der Grosshirnrinde in ihren Prinzipien dargestellt auf Grund des Zellenbaues. Barth.

Buracas, G. T. and Boynton, G. M. (2007). The effect of spatial attention on contrast response functions in human visual cortex. Journal of Neuroscience, 27(1):9397.

Buschman, T. J. and Miller, E. K. (2007). Top-down versus bottom-up control of attention in the prefrontal and posterior parietal cortices. Science, 315(5820):18601862.

Cameron, L. E., Tai, J. C., and Carrasco, M. (2002). Covert attention affects the psychometric function of contrast sensitivity. Vision Research, 42:949-967.

Carandini, M. and Ferster, D. (1997). A tonic hyperpolarization underlying contrast adaptation in cat visual cortex. Science, 276:949-952. 
Carandini, M., Heeger, D. J., and Movshon, J. A. (1997). Linearity and normalization in simple cells of the macaque primary visual cortex. Journal of Neuroscience, 17:8621-8644.

Carrasco, M., Ling, S., and Read, S. (2004). Attention alters appearance. Nat Neurosci, 7(3):308-313.

Carrasco, M., Penpeci-Talgar, C., and Eckstein, M. (2000). Spatial covert attention increases contrast sensitivity along the csf: Support for signal enhancement. Vision Research, 40:1203-1215.

Chaudhuri, A. (1990). Modulation of the motion aftereffect by selective attention. Nature, 344:60-62.

Chelazzi, L., Miller, E. K., Duncan, J., and Desimone, R. (1993). A neural basis for visual search in the inferior temporal cortex. Nature, 363:345-347.

Chelazzi, L., Miller, E. K., Duncan, J., and Desimone, R. (2001). Responses of neurons in macaque area V4 during memory-guided visual search. Cerebral Cortex, 11(8):761-772.

Clifford, C. W. G. (2002). Perceptual adaptation: motion parallels orientation. Trends in Cognitive Sciences, 6:136-143.

Clifford, C. W. G. and Rhodes, G. (2005). Fitting the mind to the world - Adaptation and after-effects in high level vision. Number 1. Oxford University Press.

Clifford, C. W. G. and Wenderoth, P. (1999). Adaptation to temporal modulation can enhance differential speed sensitivity. Vision Research, 39:4324-4331.

Clifford, C. W. G., Wenderoth, P., and Spehar, B. (2000). A functional angle on some after-effects in cortical vision. Proc. R. Soc. Lond. B, 267:1705-1710.

Compte, A. and Wang, X.-J. (2006). Tuning curve shift by attention modulation in cortical neurons: a computational study of its mechanisms. Cerebral Cortex, 16:761-778.

Connor, C. E., Gallant, J. L., Preddie, D. C., and Van Essen, D. C. (1996). Responses in area V4 depend on the spatial relationship between stimulus and attention. Journal of Neurophysiology, 75:1306-1308. 
Connor, C. E., Preddie, D. C., Gallant, J. L., and Van Essen, D. C. (1997). Spatial attention effects in macaque area V4. Journal of Neuroscience, 17(9):3201-3214.

Cook, E. P. and Maunsell, J. H. R. (2002). Attentional modulation of behavioral performance and neuronal responses in middle temporal and ventral intraparietal areas of macaque monkey. Journal of Neuroscience, 22:1994-2004.

Crowder, N. A., Price, N. S. C., Hietanen, M. A., Dreher, B., Clifford, C. W. G., and Ibbotson, M. R. (2005). Relationship between contrast adaptation and orientation tuning in V1 and V2 of cat visual cortex. Journal of Neurophysiology, 95:271283.

Culham, J. C., Dukelow, S. P., Vilis, T., Hassard, F. A., Gati, J. S., Menon, R. S., and Goodale, M. A. (1999). Recovery of fmri activation in motion area MT following storage of the motion aftereffect. Journal of Neurophysiology, 81:388-393.

de la Rocha, J., Doiron, B., Shea-Brown, E., Josic, K., and Reyes, A. (2007). Correlation between neural spike trains increases with firing rate. Nature, 448(7155):802-806.

Dean, I., Harper, N. S., and McAlpine, D. (2005). Neural population coding of sound level adapts to stimulus statistics. Nat Neurosci, 8(12):1684-1689.

Desimone, R. and Duncan, J. (1995). Neural mechanisms of selective visual attention. Annual Review of Neuroscience, 18:193-222.

Ditterich, J., Mazurek, M. E., and Shadlen, M. N. (2003). Microstimulation of visual cortex affects the speed of perceptual decisions. Nat Neurosci, 6:891-898.

Douglas, R. J. and Martin, K. A. C. (2004). Neuronal circuits of the neocortex. Annual Review of Neuroscience, 27:419-451.

Dragoi, V., Sharma, J., Miller, E. K., and Sur, M. (2002). Dynamics of neuronal sensitivity in visual cortex and local feature discrimination. Nat Neurosci, 5:883891.

Dragoi, V., Sharma, J., and Sur, M. (2000). Adaptation-induced plasticity of orientation tuning in adult visual cortex. Neuron, 28:287-298. 
Dubner, R. and Zeki, S. (1971). Response properties and receptive fields of cells in an anatomically defined region of the superior temporal sulcus in the monkey. Brain Research, 35(2):528-32.

Duncan, J. (1984). Selective attention and the organization of visual information. Journal of Experimental Psychology, 113:501-517.

Fairhall, A. L., Lewen, G. D., Bialek, W., and de Ruyter van Steveninck, R. R. (2001). Efficiency and ambiguity in an adaptive neural code. Nature, 412:787792.

Fang, F., Murray, S. O., Kersten, D., and He, S. (2005). Orientation-tuned fmri adaptation in human visual cortex. Journal of Neurophysiology, 94:4188-4195.

Felleman, D. J. and Van Essen, D. C. (1991). Distributed hierarchical processing in the primate cerebral cortex. Cerebral Cortex, 1(1):1-a-47.

Felsen, G., Shen, Y.-s., Yao, H., Spor, G., Li, C., and Dan, Y. (2002). Dynamic modification of cortical orientation tuning mediated by recurrent connections. Neuron, 36:945-954.

Ferrera, V., Rudolph, K., and Maunsell, J. (1994a). Responses of neurons in the parietal and temporal visual pathways during a motion task. Journal of Neuroscience, 14:6171-6186.

Ferrera, V. P., Nealey, T., and Maunsell, J. H. R. (1994b). Responses in the macaque visual area V4 following inactivation of the parvocellular aand magnocellular LGN pathaways. Journal of Neuroscience, 14(2080-2088).

Fries, P., Reynolds, J. H., Rorie, A. E., and Desimone, R. (2001). Modulation of oscillatory neuronal synchronization by selective visual attention. Science, 291(5508):1560-1563.

Ghose, G. M. and Maunsell, J. H. R. (2002). Attentional modulation in visual cortex depends on task timing. Nature, 419:616-620.

Giaschi, D., Douglas, R., Marlin, S., and Cynader, M. (1993). The time course of direction-selective adaptation in simple and complex cells in cat striate cortex. Journal of Neurophysiology, 70:2024-2034. 
Gibson, J. J. (1937). Adaptation, after-effect and contrast in the perception of tilted lines: Ii. simultaneous contrast and the area restriction of the after-effect. Journal of Experimental Psychology, 20:553-569.

Gibson, J. J. and Radner, M. (1937). Adaptation, after-effect and contrast in the perception of tilted lines: I. quantitative studies. Journal of Experimental Psychology, 20:453-467.

Glimcher, P. W. (2002). Decisions, decisions, decisions: Choosing a biological science of choice;. Neuron, 36(2):323-332.

Gottlieb, J. P., Kusunoki, M., and Goldberg, M. E. (1998). The representation of visual salience in monkey parietal cortex. Nature, 391:481-484.

Groh, J. M., Born, R. T., and Newsome, W. T. (1997). How is a sensory map read out? effects of microstimulation in visual area MT on saccades and smooth pursuit eye movements. Journal of Neuroscience, 17(11):4312-4330.

Guo, K., Benson, P. J., and Blakemore, C. (2004). Pattern motion is present in $\mathrm{v} 1$ of awake but not anaesthetized monkeys. European Journal of Neuroscience, 19:1055-1066.

Haenny, P. E., Maunsell, J. H. R., and Schiller, P. H. (1988). State dependent activity in monkey visual cortex. Experimental Brain Research, 69:245-259.

Haenny, P. E. and Schiller, P. H. (1988). State dependent activity in monkey visual cortex i. Experimental Brain Research, 69:225-244.

Hanes, D. P., Thompson, K. G., and Schall, J. D. (1995). Relationship of presaccadic activity in frontal eye field and supplementary eye field to saccade initiation in macaque: Poisson spike train analysis. Experimental Brain Research, 103:8596.

He, S., Cohen, E. R., and Hu, X. (1998). Close correlation between activity in brain area MT/V5 and the perception of a visual motion aftereffect;. Current Biology, 8(22):1215-1218.

Heuer, H. W. and Britten, K. H. (2002). Contrast dependence of response normalization in area MT of the rhesus macaque. Journal of Neurophysiology, 88(6):33983408 . 
Hol, K. and Treue, S. (2001). Different populations of neurons contribute to the detection and discrimination of visual motion. Vision Research, 41:685-689.

Hubel, D. H. and Wiesel, T. N. (1962). Receptive fields, binocular interaction and functional architecture in the cat's visual cortex. The Journal of Physiology Online, 160(1):106-154.

Hubel, D. H. and Wiesel, T. N. (1968). Receptive fields and functional architecture of monkey striate cortex. The Journal of Physiology Online, 195(1):215-243.

Huk, A. C., Dougherty, R. F., and Heeger, D. J. (2002). Retinotopy and functional subdivision of human areas MT and MST. Journal of Neuroscience, 22(16):71957205.

Huk, A. C., Ress, D., and Heeger, D. J. (2001). Neuronal basis of the motion aftereffect reconsidered;. Neuron, 32(1):161-172.

Itti, L. and Koch, C. (2001). Computational modelling of visual attention. Nature Reviews Neuroscience, 2:194-203.

Itti, L., Rees, G., and Tsotsos, J. K. (2005). Neurobiology of Attention. Elsevier Academic Press.

Janssen, P. and Shadlen, M. N. (2005). A representation of the hazard rate of elapsed time in macaque area LIP. Nat Neurosci, 8:234-241.

Kandel, E., Schwartz, J. H., and Jessell, T. M. (2000). Principles of Neural Sciences. McGraw-Hill.

Kastner, S., De Weerd, P., Desimone, R., and Ungerleider, L. G. (1998). Mechanisms of directed attention in the human extrastriate cortex as revealed by functional mri. Science, 282(5386):108-111.

Kastner, S., Pinsk, M. A., De Weerd, P., Desimone, R., and Ungerleider, L. G. (1999). Increased activity in human visual cortex during directed attention in the absence of visual stimulation. Neuron, 22:751-761.

Kawaguchi, Y. (1995). Physiological subgroups of nonpyramidal cells with specific morphological characteristics in layer ii/iii of rat frontal cortex. Journal of Neuroscience, 15(4):2638-2655. 
Klein, R. D. (2000). Inhibition of return. Trends in Cognitive Sciences, 4(4):138147.

Kohn, A. (2007). Visual adaptation: physiology, mechanisms, and functional benefits. Journal of Neurophysiology, 97:3155-3164.

Kohn, A. and Movshon, J. A. (2003). Neuronal adaptation to visual motion in area MT of the macaque. Neuron, 39:681-691.

Kohn, A. and Movshon, J. A. (2004). Adaptation changes the direction tuning of macaque MT neurons. Nat Neurosci, 7:764-772.

Kreiman, G., Hung, C. P., Kraskov, A., Quiroga, R. Q., Poggio, T., and DiCarlo, J. J. (2006). Object selectivity of local field potentials and spikes in the macaque inferior temporal cortex. Neuron, 49(3):433-445.

Krekelberg, B., van Wezel, R. J. A., and Albright, T. D. (2006). Adaptation in macaque MT reduces perceived speed and improves speed discrimination. Journal of Neurophysiology, 95:255-270.

Latham, P. E. and Nirenberg, S. (2005). Synergy, redundancy, and independence in population codes, revisited. Journal of Neuroscience, 25(21):5195-5206.

Lennie, P. (2003). The cost of cortical computation. Current Biology, 13:493-497.

Leopold, D., Rhodes, G., Müller, K., and Jeffery, L. (2005). The dynamics of visual adaptation to faces. Proceedings of the Royal Society B: Biological Sciences, 272(1566):897-904.

Leopold, D. A., O’Toole, A., Vetter, T., and Blanz, V. (2001). Prototype-reference shape encoding revealed by high-level aftereffects. Nat Neurosci, 4(1):89-94.

Levinson, E. and Sekuler, R. (1976). Adaptation alters perceived direction of motion. Vision Research, 16:779-780.

Liu, J. and Newsome, W. T. (2003). Functional organization of speed tuned neurons in visual area MT. J Neurophysiol, 89(1):246-256.

Logothetis, N. K. (2003). The underpinnings of the bold functional magnetic resonance imaging signal. Journal of Neuroscience, 23(10):3963-3971. 
Logothetis, N. K., Pauls, J., Augath, M., Trinath, T., and Oeltermann, A. (2001). Neurophysiological investigation of the basis of the fmri signal. Nature, 412(6843):150-157.

Lu, Z.-L. and Dosher, B. A. (1998). External noise distinguishes attention mechanisms. Vision Research, 38(9):1183-1198.

Luck, S. J., Chelazzi, L., Hillyard, S. A., and Desimone, R. (1997). Neural mechanisms of spatial selective attention in areas V1, V2, and V4 of macaque visual cortex. Journal of Neurophysiology, 77:24-42.

Ma, W. J., Beck, J. M., Latham, P. E., and Pouget, A. (2006). Bayesian inference with probabilistic population codes. Nat Neurosci, 9(11):1432-1438.

Markram, H., Toledo-Rodriguez, M., Wang, Y., Gupta, A., Silberberg, G., and Wu, C. (2004). Interneurons of the neocortical inhibitory system. Nature Reviews Neuroscience, 5:793-807.

Marlin, S. G., Hasan, S. J., and Cynader, M. S. (1988). Direction-selective adaptation in simple and complex cells in cat striate cortex. Journal of Neurophysiology, 59:1314-1330.

Martínez-Trujillo, J. C. and Treue, S. (2002). Attentional modulation strength in cortical area MT depends on stimulus contrast. Neuron, 35:365-370.

Martínez-Trujillo, J. C. and Treue, S. (2004). Feature-based attention increases the selectivity of population responses in primate visual cortex. CurrentBiology, 14:744-751.

Masuda, N. and Aihara, K. (2003). Ergodicity of spike trains: When does trial averaging make sense? Neural Computation, 15(6):1341-1372.

Mather, G. (1998). The Motion Aftereffect: A Modern Perspective. MIT Press.

Maunsell, J. H. R. and Cook, E. P. (2002). The role of attention in visual processing. Phil. Trans. R. Soc. Lond. B, 357:1063-1072.

Maunsell, J. H. R. and Treue, S. (2006). Feature-based attention in visual cortex. Trends in Neurosciences, 29(6):317-322. 
Maunsell, J. H. R. and Van Essen, D. C. (1983). Functional properties of neurons in middle temporal visual area of the macaque monkey. i. selectivity for stimulus dierection, speed, and orientation. Journal of Neurophysiology, 49(5):1127-1147.

McAdams, C. J. and Maunsell, J. H. R. (1999). Effects of attention on orientationtuning functions of single neurons in macaque cortical area V4. Journal of Neuroscience, 19:431-441.

McAdams, C. J. and Maunsell, J. H. R. (2000). Attention to both space and feature modulates neuronal responses in macaque area V4. Journal of Neurophysiology, $83: 1751-1755$.

McCarthy, J. E. (1993). Directional adaptation effects with contrast modulated stimuli. Vision Research, 33:2653-2662.

Mitchell, J. F., Sundberg, K. A., and Reynolds, J. H. (2007). Differential attentiondependent response modulation acrosss cell classes in macaque vusual area V4. Neuron, 55:131-141.

Moore, T. and Armstrong, K. M. (2003). Selective gating of visual signals by microstimulation of frontal cortex. Nature, 421(6921):370-373.

Moore, T. and Fallah, M. (2004). Microstimulation of the frontal eye field and its effects on covert spatial attention. Journal of Neurophysiology, 91:152-162.

Moran, J. and Desimone, R. (1985). Selective attention gates visual processing in the extrastriate cortex. Science, 229(4715):782-784.

Motter, B. (1994). Neural correlates of feature selective memory and pop-out in extrastriate area V4. Journal of Neuroscience, 14:2190-2199.

Motter, B. C. (1993). Focal attention produces spatially selective processing in visual cortical areas V1, V2, and V4 in the presence of competing stimuli. Journal of Neurophysiology, 70:909-919.

Movshon, J. A., Adelson, E. H., Gizzi, M. S., and Newsome, W. T. (1985). The analysis of moving visual patterns, volume 54, pages 117 - 150. Pontificiae Academiae Scientarium Scripta Varia.

Movshon, J. A. and Lennie, P. (1979). Pattern-selective adaptation in visual cortical neurones. Nature, 278:850-852. 
Müller, J. R., Metha, A. B., Krauskopf, J., and Lennie, P. (1999). Rapid adaptation in visual cortex to the structure of images. Science, 285:1405-1408.

Müller, J. R., Metha, A. B., Krauskopf, J., and Lennie, P. (2001). Information conveyed by onset transients in responses of striate cortical neurons. Journal of Neuroscience, 21(17):6978-6990.

Murray, S. O. and Wojciulik, E. (2004). Attention increases neural selectivity in the human lateral occipital complex. Nat Neurosci, 7:70-74.

Nelson, S. (1991). Temporal interactions in the cat visual system. i. orientationselective suppression in the visual cortex. Journal of Neuroscience, 11:344-356.

Newsome, W. and Pare, E. (1988). A selective impairment of motion perception following lesions of the middle temporal visual area (MT). Journal of Neuroscience, 8(6):2201-2211.

Nichols, M. J. and Newsome, W. T. (2002). Middle temporal visual area microstimulation influences veridical judgments of motion direction. Journal of Neuroscience, 22:9530-9540.

Nowak, L. G., Azouz, R., Sanchez-Vives, M. V., Gray, C. M., and McCormick, D. A. (2003). Electrophysiological classes of cat primary visual cortical neurons in vivo as revealed by quantitative analyses. Journal of Neurophysiology, 89(3):15411566.

O’Connor, D. H., Fukui, M. M., Pinsk, M. A., and Kastner, S. (2002). Attention modulates responses in the human lateral geniculate nucleus. Nat Neurosci, 5(11):1203-1209.

Osborne, L. C., Bialek, W., and Lisberger, S. G. (2004). Time course of information about motion direction in visual area MT of macaque monkeys. Journal of Neuroscience, 24:3210-3222.

O'Toole, B. and Wenderoth, P. (1977). The tilt illusion: Repulsion and attraction effects in the oblique meridian;. Vision Research, 17(3):367-374.

Perrone, J. A. and Thiele, A. (2001). Speed skills: measuring the visual speed analyzing properties of primate MT neurons. Nat Neurosci, 4:526-532. 
Pessoa, L., Kastner, S., and Ungerleider, L. G. (2003). Neuroimaging studies of attention: From modulation of sensory processing to top-down control. Journal of Neuroscience, 23(10):3990-3998.

Petersen, S. E., Baker, J. F., and Allman, J. M. (1985). Direction-specific adaptation in area MT of the owl monkey. Brain Research, 346:146-150.

Posner, M. I. and Cohen, Y. (1984). Components of visual orienting. In Bouma, H. and Bouwhius, D., editors, Attention and Perforance Vol. X, volume 10. Erlbaum.

Posner, M. I., Snyder, C. A., and Davidson, B. J. (1980). Orienting of attention. Quaterly Journal of Experimental Psychology, 109(32):3-25.

Pouget, A., Dayan, P., and Zemel, R. (2000). Information processing with population codes. Nature Reviews Neuroscience, 1:125-132.

Priebe, N. J., Cassanello, C. R., and Lisberger, S. G. (2003). The neural representation of speed in macaque area MT/V5. Journal of Neuroscience, 23:5650-5661.

Purushothaman, G. and Bradley, D. C. (2005). Neural population code for fine perceptual decisions in area MT. Nat Neurosci, 8:99-106.

Rensink, R. A., O’Regan, J. K., and Clark, J. J. (1997). To see or not to see:. the need for attention to perceive changes in scenes. Psychological Science, 8(5):368-373.

Reynolds, J. H., Chelazzi, L., and Desimone, R. (1999). Competitive mechanisms subserve attention in macaque areas V2 and V4. Journal of Neuroscience, 19:1736-1753.

Reynolds, J. H. and Desimone, R. (1999). The role of neural mechanisms of attention in solving the binding problem. Neuron, 24:19-29.

Reynolds, J. H. and Desimone, R. (2003). Interacting roles of attention and visual salience in V4. Neuron, 37(5):853-863.

Reynolds, J. H., Pasternak, T., and Desimone, R. (2000). Attention increases sensitivity of V4 neurons. Neuron, 26:703-714.

Rezec, A., Krekelberg, B., and Dobkins, K. R. (2004). Attention enhances adaptability: evidence from motion adaptation experiments. Vision Research, 44:30353044. 
Rizzolatti, G. (1983). Mechanisms of selective attention in mammals. In Ewert, J. P., Capranica, R. R., and Ingle, D. J., editors, Advances in vertebrate neuropathology, pages 261-297. Plenum Press.

Roelfsema, P. R., Lamme, V. A. F., and Spekreijse, H. (1998). Object-based attention in the primary visual cortex of the macaque monkey. Nature, 395:376-381.

Roerig, B., Chen, B., and Kao, J. P. Y. (2003). Different inhibitory synaptic input patterns in excitatory and inhibitory layer 4 neurons of ferret visual cortex. Cerebral Cortex, 13:350-363.

Rossi, A. F. and Paradiso, M. A. (1995). Feature-specific effects of selective visual attention. Vision Research, 35(5):621-634.

Saenz, M., Buracas, G. T., and Boynton, G. M. (2002). Global effects of featurebased attention in human visual cortex. Nat Neurosci, 5(7):631-632.

Salzman, C. and Newsome, W. (1994). Neural mechanisms for forming a perceptual decision. Science, 264(5156):231-237.

Salzman, C. D., Britten, K. H., and Newsome, W. T. (1990). Cortical microstimulation influences perceptual judgements of motion direction. Nature, 346(6280):174-177.

Sanchez-Vives, M. V., Nowak, L. G., and McCormick, D. A. (2000). Membrane mechanisms underlying contrast adaptation in cat area 17 in vivo. Journal of Neuroscience, 20:4267-4285.

Sanger, T. D. (2003). Neural population codes. Current Opinion in Neurobiology, 13:238-249.

Saul, A. B. (1995). Adaptation aftereffects in single neurons of cat visual cortex: Response timing is retarded by adaptation. Visual Neuroscience, 12:191-205.

Schmolesky, M. T., Wang, Y., Hanes, D. P., Thompson, K. G., Leutgeb, S., Schall, J. D., and Leventhal, A. G. (1998). Signal timing across the macaque visual system. Journal of Neurophysiology, 79:3272-3278.

Schrater, P. R. and Simoncelli, E. P. (1998). Local velocity representation: evidence from motion adaptation. Vision Research, 38:3899-3912. 
Schwartz, O., Hsu, A., and Dayan, P. (2007). Space and time in visual cortex. Nature Reviews Neuroscience, 8:522-535.

Sekuler, R. and Littlejohn, J. (1974). Tilt aftereffect following very brief exposures. Vision Research, 14:151-152.

Serences, J. T. and Boynton, G. M. (2007). Feature-based attentional modulation in the absence of direct visual simulation. Neuron, 55:301-312.

Sheinberg, D. L. and Logothetis, N. K. (2001). Noticing familiar objects in real world scenes: The role of temporal cortical neurons in natural vision. Journal of Neuroscience, 21(4):1340-1350.

Shinomoto, S., Shima, K., and Tanji, J. (2003). Differences in spiking patterns among cortical neurons. Neural Computation, 15:2823-2842.

Shoham, S., O'Connor, D. H., and Segev, R. (2006). How silent is the brain: is there a 'dark matter' problem in neuroscience? Journal of Comparative Physiology A: Neuroethology, Sensory, Neural, and Behavioral Physiology, V192(8):777-784.

Silver, M. A., Ress, D., and Heeger, D. J. (2007). Neural correlates of sustained spatial attention in human early visual cortex. Journal of Neurophysiology, 97(1):229-237.

Simoncelli, E. P. and Heeger, D. J. (1998). A model of neuronal responses in visual area MT. Vision Research, 38:743-761.

Simons, D. J. (2000). Attentional capture and inattentional blindness. Trends in Cognitive Sciences, 4(4):147-155.

Simons, D. J. and Chabris, C. F. (1999). Gorillas in our midst: sustained inattentional blindness for dynamic events. Perception, 28:1059-1074.

Simons, D. J. and Rensink, R. A. (2005). Change blindness: past, present and future. Trends in Cognitive Sciences, 9(1).

Skottun, B. C., Bradley, A., Sclar, G., Ohzawa, I., and Freeman, R. D. (1987). The effects of contrast on visual orientation and spatial frequency discrimination: a comparison of single cells and behavior. Journal of Neurophysiology, 57(3):773786. 
Spitzer, H., Desimone, R., and Moran, J. (1988). Increased attention enhances both behavioral and neuronal performance. Science, 240:338-340.

Stettler, D. D., Das, A., Bennett, J., and Gilbert, C. D. (2002). Lateral connectivity and contextual interactions in macaque primary visual cortex;. Neuron, 36(4):739-750.

Suzuki, S. (2001). Attention-dependent brief adaptation to contour orientation: a high-level aftereffect for convexity? Vision Research, 41:3883-3902.

Swindale, N. V. (1998). Orientation tuning curves: empirical description and estimation of parameters. Biological Cybernetics, 78:45-56.

Taylor, K., Mandon, S., Freiwald, W. A., and Kreiter, A. K. (2005). Coherent oscillatory activity in monkey area V4 predicts successful allocation of attention. Cerebral Cortex, 15(9):1424-1437.

Theeuwes, J. (1991). Exogenous and endogenous control of attention: The effect of visual onsets and offsets. Perception \& Psychophysics, 49(1):83-90.

Thiele, A., Dobkins, K. R., and Albright, T. D. (2000). Neural correlates of contrast detection at threshold. Neuron, 26:715-724.

Thompson, P. (1981). Velocity after-effects: The effects of adaptation to moving stimuli on the perception of subsequently seen moving stimuli;. Vision Research, 21(3):337-345.

Tinsley, C. J., Webb, B. S., Barraclough, N. E., Vincent, C. J., Parker, A., and Derrington, A. M. (2003). The nature of V1 neural responses to $2 \mathrm{~d}$ moving patterns depends on receptive-field structure in the marmoset monkey. Journal of Neurophysiology, 90:930-937.

Tolias, A. S., Moore, T., Smirnakis, S. M., Tehovnik, E. J., Siapas, A. G., and Schiller, P. H. (2001a). Eye movements modulate visual receptive fields of V4 neurons. Neuron, 29:757-767.

Tolias, A. S., Smirnakis, S. M., Augath, M. A., Trinath, T., and Logothetis, N. K. (2001b). Motion processing in the macaque: Revisited with functional magnetic resonance imaging. Journal of Neuroscience, 21:8594-8601. 
Tootell, R. B. H., Hadjikhani, N., Hall, e. K., Marrett, S., Vanduffel, W., Vaughan, J., and Dale, A. M. (1998). The retinotopy of visual spatial attention. Neuron, 21(6):1409-1422.

Towe, A. L. and Harding, G. W. (1970). Extracellular microelectrode sampling bias. Experimental Neurology, 29(2):366-381.

Treisman, A. and Gelade, G. (1980). A feature-integration theory of attention. Cognitive Psychology, 12:97-136.

Treue, S., Hol, K., and Rauber, H.-J. (2000). Seeing multiple directions of motion physiology and psychophysics. Nat Neurosci, 3:270-276.

Treue, S. and Martínez-Trujillo, J. C. (1999). Feature-based attention influences motion processing gain in macaque visual cortex. Nature, 399:575-579.

Treue, S. and Martínez-Trujillo, J. C. (2003). Cognitive physiology: Moving the mind's eye before the head's eye. Current Biology, 13:R442-R444.

Treue, S. and Martínez-Trujillo, J. C. (2007). Attending to features inside and outside the spotlight of attention. Neuron, 55:174-176.

Treue, S. and Maunsell, J. H. R. (1996). Attentional modulation of visual motion processing in cortical areas MT and MST. Nature, 382(6591):539-541.

Treue, S. and Maunsell, J. H. R. (1999). Effects of attention on the processing of motion in macaque middle temporal and medial superior temporal visual cortical areas. Journal of Neuroscience, 19:7591-7602.

Tsao, D. Y., Freiwald, W. A., Knutsen, T. A., Mandeville, J. B., and Tootell, R. B. H. (2003). Faces and objects in macaque cerebral cortex. Nat Neurosci, 6(9):989995.

Valdes-Sosa, M., Cobo, A., and Pinilla, T. (1998). Transparent motion and objectbased attention. Cognition, 66.

Valdes-Sosa, M., Cobo, A., and Pinilla, T. (2000). Attention to object files defined by transparent motion. Journal of Experimental Psychology, 26:488-505.

van de Grind, W. A., van der Smagt, M. J., and Verstraten, F. A. J. (2004). Storage for free: a surprising property of a simple gain-control model of motion aftereffects. Vision Research, 44:2269-2284. 
Van Wezel, R. J. A. and Britten, K. H. (2002). Motion adaptation in area MT. Journal of Neurophysiology, 88:3469-3476.

Verstraten, F. A. J. (1996). On the ancient history of the direction of the motion aftereffect. Perception, 25:1177-88.

Vogels, R. and Orban, G. A. (1994). Activity of inferior temporal neurons during orientation discrimination with successively presented gratings. Journal of Neurophysiology, 71(4):1428-1451.

Wannig, A., Rodriguez, V., and Freiwald, W. A. (2007). Attention to surfaces modualtes motion processing in extrastraite area MT. Neuron, 54:639-651.

Webster, M. A., Kaping, D., Mizokami, Y., and Duhamel, P. (2004). Adaptation to natural facial categories. Nature, 428:557-561.

Wenderoth, P. and Johnstone, S. (1988). The different mechanisms of the direct and indirect tilt illusions;. Vision Research, 28(2):301-312.

Williford, T. and Maunsell, J. H. R. (2006). Effects of spatial attention on contrast response functions in macaque area V4. Journal of Neurophysiology, 96(1):4054.

Wohlgemuth (1911). On the after-effect of seen movement. British Journal of Psychology, 1:1-117.

Womelsdorf, T., Anton-Erxleben, K., Pieper, F., and Treue, S. (2006a). Dynamic shifts of visual receptive fields in cortical area MT by spatial attention. Nat Neurosci, 9(9):1156-1160.

Womelsdorf, T. and Fries, P. (2007). The role of neuronal synchronization in selective attention. Current Opinion in Neurobiology, 17:154-160.

Womelsdorf, T., Fries, P., Mitra, P. P., and Desimone, R. (2006b). Gamma-band synchronization in visual cortex predicts speed of change detection. Nature, 439:733-736.

Yantis, S. and Serences, J. T. (2003). Cortical mechanisms of space-based attentional control. Current Opinion in Neurobiology, 13.

Yeshurun, Y. and Carrasco, M. (1998). Attention improves or impairs visual performance by enhancing spatial resolution. Nature, 396:72-75. 


\section{Chapter 6}

\section{Curriculum Vitae}

\section{Florian Pieper}

\section{Personal Details}

Date of birth: $\quad$ 14th December 1972

Place of birth: $\quad$ Brake /Unterweser, Germany

Martial status: $\quad$ married, 1 child

\section{Education}

since $01 / 02$

Ph.D. student at the German Primate Center, Göttingen

Advisor: Prof. Dr. S.Treue

12/99-08/01 Diploma in Biology, University of Göttingen, German Primate Center

Thesis: Neuronal activity in the Mesencephalon and the bordering Pons during Vokalization. - A single-unit study in the squirrel monkey (Saimiri sciureus)

Advisor: Prof. Uwe Jürgens

04/96-11/99 Graduate studies in Biology, University of Göttingen,

Germany

10/93-03/96 Undergraduate studies in Biology, University of Oldenburg in Oldbg., Germany 


\section{Relevant experience}

10/04 Participant in the "Analysis and Models in Neurophysiology"course. German Neuroscience Society, University of Freiburg, Germany

07/04 Participant in the Summer Course "Computational Neuroscience: Vision" at the Cold Spring Harbor Laboratories, Cold Spring Harbor, NY, USA

09/03 Participant in the "Computational Neurosciences"-Course. German Neuroscience Society, Max-Planck-Institute for Non-linear Dynamics , Göttingen, Germany

01/99-12/01 Research Assistant

Dept. of Neurobiology, German Primate Center, Göttingen, Germany

Advisor: Prof. Uwe Jürgens

08/96-12/97 Research Assistant

Dept. of Neurobiology, German Primate Center, Göttingen, Germany

Advisor: Prof. Uwe Jürgens

\section{Journal publications}

$\bullet$

Pieper F., Womelsdorf T., Patzwahl D., Treue S. \& MartínezTrujillo J. C. Response saturation determines attentional modulation onset in MT neurons. Prepared for submission

Pieper, F. \& Treue, S. Brief Motion adaptation causes repulsive aftereffects. Prepared for submission.

Womelsdorf T., Anton-Erxleben K., Pieper F., \& Treue S. (2006). Dynamic shifts of visual receptive fields in cortical area MT by spatial attention, Nat Neurosci, vol.9, no.9, pp.11561160 .

Pieper F. \& Jürgens U. (2003). Neuronal activity in the inferior colliculus and bordering structures during vocalization in the squirrel monkey. Brain Research, vol.979, pp.153-164. 


\section{Published abstracts}

- $\quad$ Pieper F., Womelsdorf T., Martínez-Trujillo J. C. \& Treue S. (2007). Response saturation determines the attentional delay in MT neurons. Poster, 3rd Symposium for Computational Neuroscience, BCCN, Göttingen

Pieper F., Womelsdorf T., Martínez-Trujillo J. C. \& Treue S. (2007). Variability of attentional modulation of neurons in visual cortical area MT of rhesus monkeys., Poster, 31st Meeting of the German Neuroscience Society, Göttingen

Pieper F., Womelsdorf T., Martínez-Trujillo J. C. \& Treue S. (2006). Basic neuronal response properties shape the dynamics and strength of attentional modulation in visual cortex., 36th Meeting of the Society for Neuroscience, Atlanta, USA

Pieper F., Peters T. \& Treue S. (2005). The influence of brief motion adaptation on direction perception. Poster, 30th Annual Meeting of the German Neuroscience Community, Göttingen

Pieper F. \& Treue S. (2004). Motion adaptation occurs within a few seconds. Poster, 34th Meeting of the Society for Neuroscience, SanDiego, USA

Pieper F. \& Jürgens U. (2003). A possible vocal-audio interface in the squirrel monkey's brainstem. Poster, 29th Annual Meeting of the German Neuroscience Community, Göttingen

Pieper F. \& Jürgens U. (2002). Pre-vocal neuronal activity in the external and dorsal nuclei of the inferior colliculus (Icx; Icd). 3rd Forum of European Neuroscience, Paris, France

Katzner S., Pieper F. \& Treue S. (2002). Effects of attention and contrast on detection and discrimination of visual motion: Evidence from psychophysics. Poster, 32nd Meeting of the Society for Neuroscience, Orlando, USA

Göttingen, 18th of September, 2007 